\title{
Ontogeny of Postcranial Robusticity among Holocene Hunter-Gatherers of Southernmost Africa
}

by

Lesley Harrington

A thesis submitted in conformity with the requirements

for the degree of Doctor of Philosophy

Graduate Department of Anthropology

University of Toronto

C Copyright by Lesley Harrington 2010 


\title{
Ontogeny of Postcranial Robusticity among Holocene Hunter-Gatherers of Southernmost Africa
}

\author{
Lesley Harrington \\ Doctor of Philosophy \\ Graduate Department of Anthropology \\ University of Toronto
}

2010

\begin{abstract}
Ontogenetic patterns in postcranial robusticity are analysed in the skeletons of eighty-two juvenile Later Stone Age hunter-gatherers with estimated ages at death ranging from infancy to late adolescence. Robusticity is quantified from cross-sectional geometric properties of diaphyses at sixteen sites throughout the skeleton, using radiography and casting methods. Skeletal growth profiles for strength measures are analysed with locally-weighted regression and additional curve fitting to evaluate developmental trajectories. Ethnographic accounts of infancy and childhood in modern Ju/'hoansi (Khoesan) communities provide a culturally-relevant interpretive framework for evaluating skeletal correlates of behaviour. This research demonstrates that bone strength properties can be functionally linked to behaviour among juveniles toward the reconstruction of patterns of physical activity among prehistoric children.

Postcranial strength measures vary predictably with age such that developmental patterns and timing in appositional growth can be assessed reliably. The onset of walking among Later Stone Age juveniles is evident by two years of age from proportional lower to upper limb strength measures. The lower limb continues to adapt to increasing loading demands especially in response to torsional forces, and bending in the medio-lateral plane; biomechanical shape measures illustrate the gradual development of the femoral pilaster.
\end{abstract}

Individual humeral strength is symmetrical with respect to the orientation maximal 
bending forces experienced throughout development, but the magnitude of the functional adaptive response is greater, on average, in the right limb with more than $70 \%$ of the sample over the age of six displaying right-side dominance. Humeral asymmetry and side dominance does not emerge until late childhood, and the majority of arm strength is accrued during adolescence, relative to the sex-combined mean values for Later Stone Age adults. Evidence for sexual dimorphism in upper limb properties is evident by late adolescence whereas sex-based patterns of mobility reflected in lower limb strength have yet to emerge. These results illustrate the developmental patterns that underlie postcranial markers of subsistence activities in Later Stone Age hunter-gatherers. 
Dedicated to the memory of Edward William Harrington (1925 - 2005) and Susan Joyce Robbins (1953 - 2007) 


\section{Acknowledgements}

A great many people provided support and encouragement toward the preparation of this thesis. I thank the curators of the skeletal remains that form the basis of this research for giving me the opportunity to study the collections under their care: Drs. Johan Binneman and Lita Webley of the Albany Museum, Grahamstown; Dr. James Brink of the National Museum, Bloemfontein; Prof. Alan Morris and Ms. Caroline Powrie, University of Cape Town; and Dr. Sarah Wurz of the Iziko South African Museum. Mrs. Gillian Bowie and her staff at Groote Schuur Hospital, Cape Town, Mrs. Mzamo and staff at Settlers' Hospital, Grahamstown, and technicians at the Hydromed Clinic in Bloemfontein contributed their skill in producing high quality radiographs, and were generous with their time and enthusiasm in accommodating this unusual project. Pat Black and Jo Fitzhenry \& Mike Dacombe provided a home-away-fromhome in Grahamstown. I offer warm thanks to Johan Binneman and Prof. Tom Huffman for sharing their enthusiasm for southern African archaeology and for including me in field excursions to sites in the Eastern Cape and in Botswana. Research in South Africa was supported by a grant from the Social Sciences and Humanities Research Council of Canada to Prof. Susan Pfeiffer.

Prof. Jay T. Stock generously shared his data for biomechanical properties of Later Stone Age adults, and was an important mentor during my early days in anthropology at the University of Toronto. Dr. Louise Humphrey provided valuable guidance toward methodological approaches for growth data. I am grateful for the continued support and encouragement of Prof. Judith Sealy.

My intellectual and professional development over the past ten years is largely attributable to the mentorship of Prof. Susan Pfeiffer. I cannot express the depth of my gratitude for Susan's support. Susan approaches her teaching and supervisory duties with exceptional dedication; her enthusiasm as an undergraduate lecturer turned me on to physical anthropology, and ever since she has guided me toward new areas of interest all the while keeping me focused on a goal. Susan supported this project in every way possible, and I can't thank her enough for the opportunity.

The "Pfeiffer Gang" was assembled variously through weekly lab meetings, providing a 
community from which I drew tremendous support. For their friendship and help along the way I thank Rhonda Bathurst, Christian Crowder, Genevieve Dewar, Olivier Dutour, Crystal Forrest and Catherine Merritt. Jaime Ginter and Helen Kurki were also wonderful travel companions, with special thanks going to Jaime for her assistance with "x-rays" and "blue rings". Other members of the Department of Anthropology whom I thank for their friendship and support toward this project include Tracy Kivell, Danielle MacDonald, Dejana Nikitovic, Mirjana Roksandic and Alexandra Sumner.

I am grateful to members of the examining committee for their efforts in helping me to improve this thesis. I have benefited from the breadth of perspective and expertise generously offered by Profs. Robert Hoppa, Esteban Parra, Michael Schillaci, and Daniel Sellen.

Finally, I am grateful to have this opportunity to formally acknowledge my friends and family who have supported me during this project, perhaps less for its intellectual content than for simply the fact that it was something that I wanted to pursue. This unconditional support is what has enabled my many years as a student, and I give special thanks to my grandparents, parents and brother for fostering my love of "school". My husband, Tolly, has given me the love and encouragement I needed to bring this project to a close, and I am grateful to the Bradford family for the generous support and guidance that has been so helpful to both of us. I dedicate this thesis to my late grandfather, Ted, who nurtured my early love of learning; and to my late cousin, Susan, who helped me to find my way as a new university student. 


\section{Table of Contents}

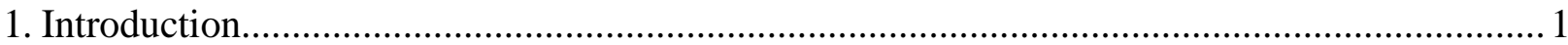

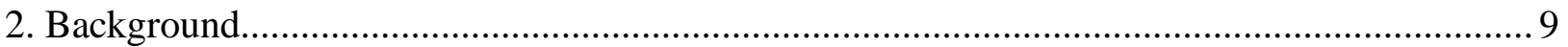

2.1 Bone functional adaptation and the role of growth.................................................

2.2 Variability in human postcranial robusticity.......................................................... 16

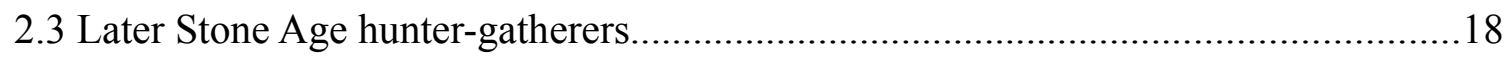

2.4 Childhood physical activity in the ethnographic record............................................24

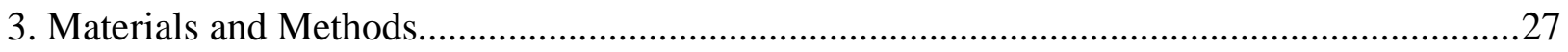

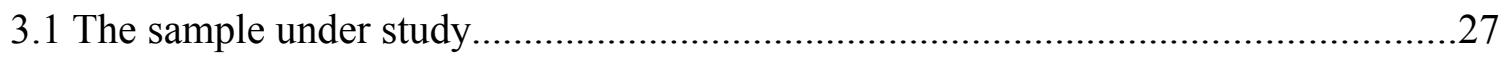

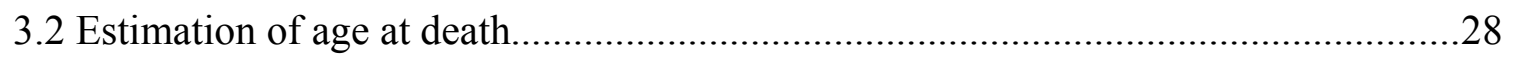

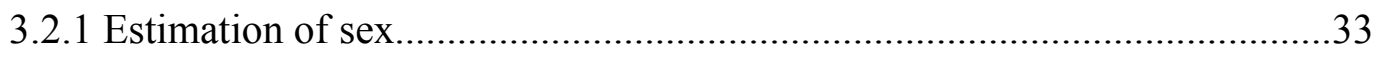

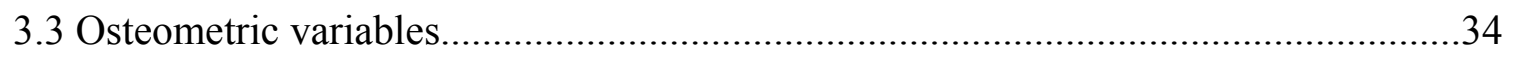

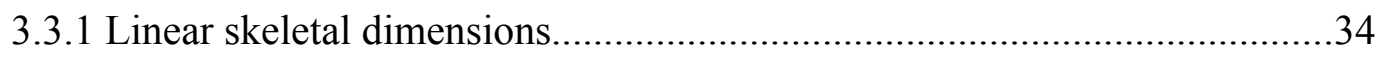

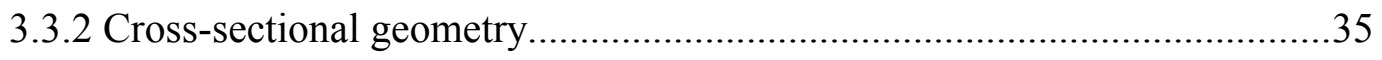

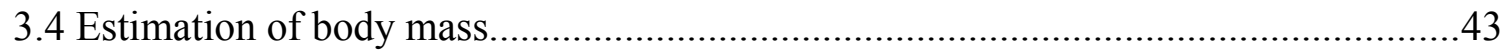

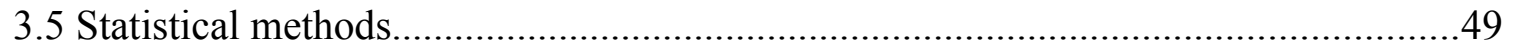

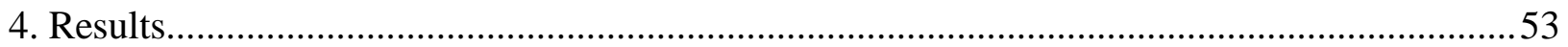

4.1 Skeletal growth profiles for postcranial robusticity................................................53

4.1.1 Cross-sectional properties of the femur...................................................54

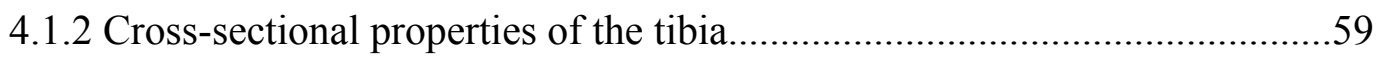

4.1.3 Cross-sectional properties of the clavicle..................................................62

4.1.4 Cross-sectional properties of the humerus...................................................64

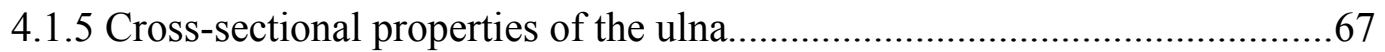

4.1.6 Summary of skeletal growth profiles for postcranial robusticity.................69

4.2 Strength proportions in the upper and lower limb..............................................

4.2.1 Lower to upper limb strength proportions..................................................71

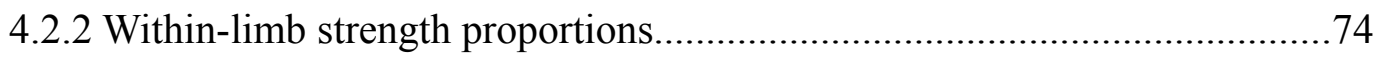

4.2.3 Summary of proportional strength measures...............................................78

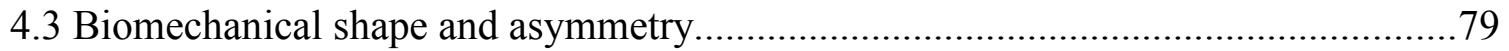

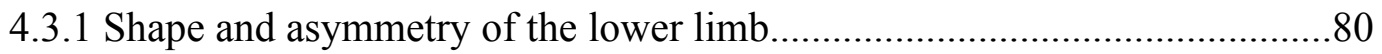

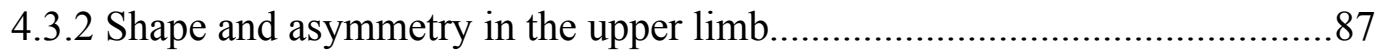

4.3.3 Summary of biomechanical shape and asymmetry.....................................95 
4.4 Body-size standardization of cross-sectional properties ...........................................97

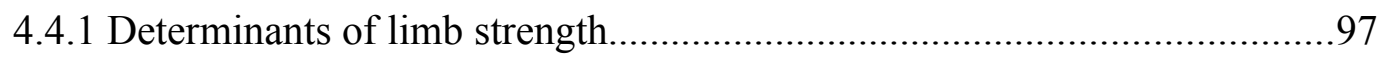

4.4.2 Size-standardized cross-sectional properties..........................................99

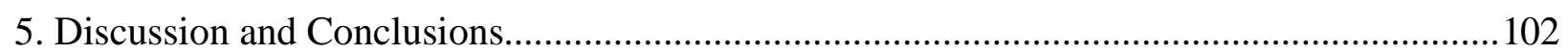

5.1 Developing locomotory behaviours and mobility levels..........................................104

5.2 Subsistence behaviours reflected in upper limb morphology....................................108

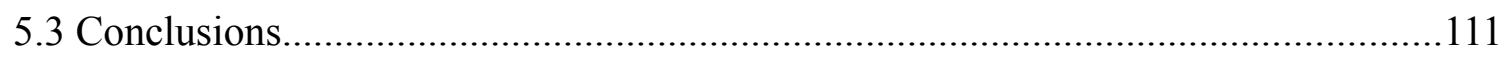




\section{List of Tables}

Table 3.1. Prediction equations for dental age from long bone lengths in Later Stone Age juveniles.

Table 3.2. Age at death estimates by age cohort in Later Stone Age juveniles..............................32

Table 3.3. Estimated sex distribution for Later Stone Age juveniles...............................................34

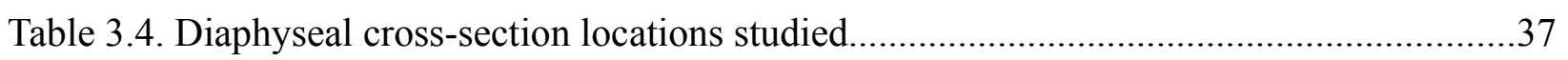

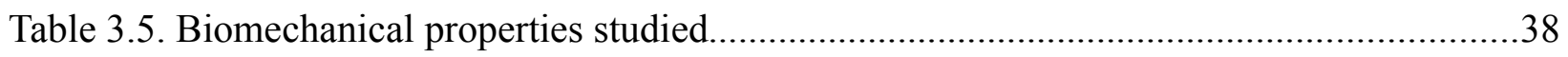

Table 3.6. Formulae for calculating biomechanical properties of a hollow beam..........................41

Table 3.7. Regression statistics for predicting body mass from femoral joint dimensions in

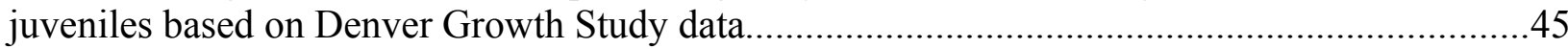

Table 3.8. Femur joint dimension prediction equations for Later Stone Age juveniles..................47

Table 3.9. Predicted body mass in Later Stone Age juveniles by age cohort.................................49

Table 4.1. R-squared values for regression of mid-shaft cross-sectional properties on age fit with

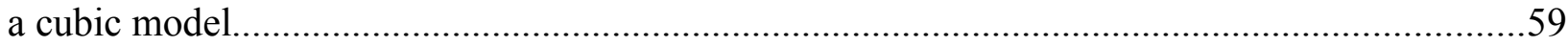

Table 4.2. Chi-square statistics for side dominance in the mid-shaft humerus..............................93

Table 4.3. Correlation coefficients for linear models of body-size related determinants of biomechanical properties. 


\section{List of Figures}

Figure 1.1. The Cape region of southernmost Africa...........................................................4

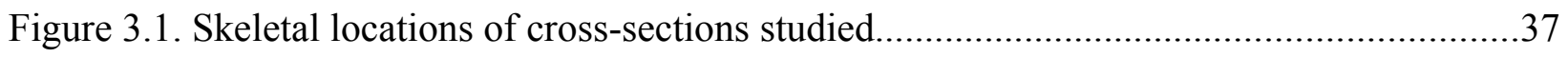

Figure 3.2. Eccentric ellipse model of a diaphyseal cross-section.........................................40

Figure 3.3. A cross-sectional reconstruction using the latex cast method................................43

Figure 3.4. Equations for the prediction of body mass in Later Stone Age juveniles in the absence

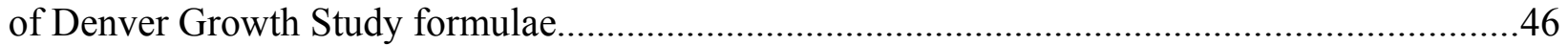

Figure 4.1. Areas (total, cortical, and medullary) of subtrochanteric $(80 \%)$ femur.....................55

Figure 4.2. Areas (total, cortical, and medullary) of the mid-shaft $(50 \%)$ femur........................55

Figure 4.3. Torsional rigidity $(\mathrm{J})$ of subtrochanteric $(80 \%)$ femur.........................................57

Figure 4.4. Torsional rigidity $(\mathrm{J})$ of mid-shaft (50\%) femur................................................57

Figure 4.5. Torsional rigidity $(\mathrm{J})$ of the mid-shaft (50\%) left femur standardized to the adult Later

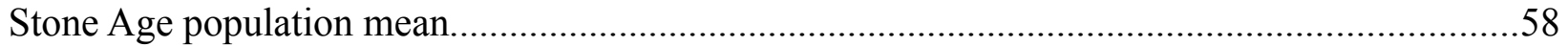

Figure 4.6. Unstandardized residuals for torsional rigidity $(\mathrm{J})$ of mid-shaft $(50 \%)$ left femur......58

Figure 4.7. Areas (total, cortical, and medullary) of tibia nutrient foramen..............................60

Figure 4.8. Areas (total, cortical, and medullary) of mid-shaft (50\%) tibia.............................60

Figure 4.9. Torsional rigidity $(\mathrm{J})$ of the tibia nutrient foramen.............................................61

Figure 4.10. Torsional rigidity $(\mathrm{J})$ of the mid-shaft (50\%) tibia...........................................61

Figure 4.11. Unstandardized residuals for torsional rigidity $(\mathrm{J})$ of mid-shaft $(50 \%)$ left femur....62

Figure 4.12. Areas (total, cortical, and medullary of the mid-shaft (50\%) clavicle....................63

Figure 4.13. Torsional rigidity $(\mathrm{J})$ of the mid-shaft $(50 \%)$ clavicle .......................................63

Figure 4.14. Unstandardized residuals for torsional rigidity $(\mathrm{J})$ of mid-shaft $(50 \%)$ right clavicle

Figure 4.15. Areas (total, cortical, and medullary) of the mid-shaft (50\%) humerus.................65

Figure 4.16. Areas (total, cortical, and medullary) of the distal (35\%) humerus........................65

Figure 4.17. Torsional rigidity $(\mathrm{J})$ of the mid-shaft (50\%) humerus........................................66

Figure 4.18. Torsional rigidity $(\mathrm{J})$ of the distal (35\%) humerus...........................................66

Figure 4.19. Torsional rigidity $(\mathrm{J})$ of the mid-shaft (50\%) right humerus standardized to the adult Later Stone Age population mean

Figure 4.20. Unstandardized residuals for torsional rigidity $(\mathrm{J})$ of mid-shaft $(50 \%)$ right humerus

Figure 4.21. Areas (total, cortical, medullary) of the mid-shaft $(50 \%)$ ulna..............................68

Figure 4.22. Torsional rigidity $(\mathrm{J})$ of the mid-shaft $(50 \%)$ ulna............................................68 
Figure 4.23. Unstandardized residuals for torsional rigidity (J) of mid-shaft (50\%) right ulna....69 Figure 4.24. Unstandardized residuals for cubic regression of right femoral to humeral proportional torsional rigidity $(\mathrm{J})$ on estimated age......

Figure 4.25. Individual proportional torsional rigidity $(\mathrm{J})$ of mid-shaft femur relative to humerus

Figure 4.26. Individual proportional torsional rigidity $(\mathrm{J})$ of mid-shaft tibia relative to humerus 74

Figure 4.27. Unstandardized residuals for cubic regression of right tibial to humeral proportional

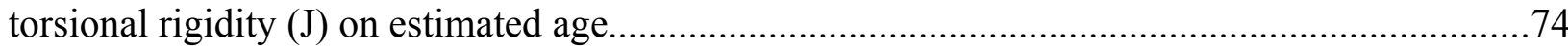

Figure 4.28. Individual proportional torsional rigidity $(\mathrm{J})$ of mid-shaft femur relative to tibia....75 Figure 4.29. Unstandardized residuals for cubic regression of left femoral to tibial proportional torsional rigidity $(\mathrm{J})$ on estimated age. .75

Figure 4.30. Individual proportional torsional rigidity $(\mathrm{J})$ of mid-shaft humerus relative to ulna 77 Figure 4.31. Unstandardized residuals for cubic regression of left humeral proportional torsional rigidity $(\mathrm{J})$ on estimated age......

Figure 4.32. Individual proportional torsional rigidity $(\mathrm{J})$ of the mid-shaft humerus relative to clavicle.

Figure 4.33. Unstandardized residuals for cubic regression of left humeral to clavicular

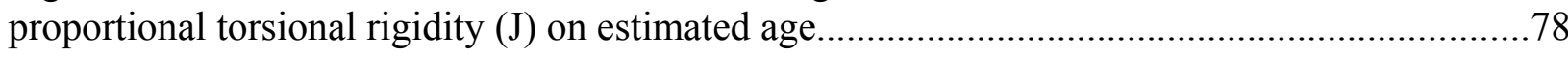

Figure 4.34. Biomechanical shape (Ix/Iy) of the subtrochanteric $(80 \%)$ femur.........................82

Figure 4.35. Biomechanical shape (Ix/Iy) of the mid-shaft $(50 \%)$ femur.................................82

Figure 4.36. Biomechanical shape (Imax/Imin) of the mid-shaft femur.................................83

Figure 4.37. Orientation of the axis of maximal bending strength (theta) of the mid-shaft (50\%)

femur.

Figure 4.38. Sex estimates for axis of maximal bending strength (Imax) of the left mid-shaft $(50 \%)$ femur .84

Figure 4.39. Percent asymmetry in torsional rigidity $(\mathrm{J})$ of subtrochanteric $(80 \%)$ femur...........85

Figure 4.40. Percent asymmetry in torsional rigidity $(\mathrm{J})$ of mid-shaft $(50 \%)$ femur...................85

Figure 4.41. Biomechanical shape (Ix/Iy) of the nutrient foramen tibia...................................86

Figure 4.42. Biomechanical shape (Ix/Iy) of the mid-shaft $(50 \%)$ tibia..................................86

Figure 4.43. Percent asymmetry in torsional rigidity of the nutrient foramen tibia....................87

Figure 4.44. Percent asymmetry in torsional rigidity of the mid-shaft (50\%) tibia....................87

Figure 4.45. Biomechanical shape (Ix/Iy) of the mid-shaft $(50 \%)$ clavicle................................8

Figure 4.46. Percent asymmetry in torsional strength $(\mathrm{J})$ of the mid-shaft clavicle....................88

Figure 4.47. Biomechanical shape (Ix/Iy) of the mid-shaft $(50 \%)$ humerus..............................89

Figure 4.48. Biomechanical shape (Ix/Iy) of the distal (35\%) humerus..................................89 
Figure 4.49. Biomechanical shape (Imax/Imin) of the mid-shaft (50\%) humerus.

Figure 4.50. Orientation of maximum bending strength (theta) of the mid-shaft (50\%) humerus 90 Figure 4.51. Sex estimates for maximum bending strength (Imax) of the left mid-shaft (50\%) humerus.

Figure 4.52. Sex estimates for maximum bending strength (Imax) of the mid-shaft $(50 \%)$ humerus. .91

Figure 4.53. Percent asymmetry in torsional rigidity of the mid-shaft (50\%) humerus..............92

Figure 4.54. Percent asymmetry in torsional rigidity of the distal $(35 \%)$ humerus.....................92

Figure 4.55. Percent asymmetry in torsional rigidity of the mid-shaft (50\%) humerus by sex.....94

Figure 4.56. Biomechanical shape (Ix/Iy) of the mid-shaft $(50 \%)$ ulna..................................94

Figure 4.57. Percent asymmetry in torsional rigidity of the mid-shaft (50\%) ulna....................94

Figure 4.58. Size-standardized areas of the mid-shaft (50\%) femur.................................... 100

Figure 4.59. Size-standardized torsional rigidity $(\mathrm{J})$ of the mid-shaft $(50 \%)$ femur...................100

Figure 4.60. Size-standardized areas of the mid-shaft (50\%) humerus..................................101

Figure 4.61. Size-standardized torsional rigidity $(\mathrm{J})$ of the mid-shaft $(50 \%)$ humerus..............101 


\section{List of Appendices}

Appendix A. Later Stone Age juveniles included in the study sample..................................130

Appendix B. Dental age prediction scatter plots and regression equations............................133

Appendix C. Femoral dimensions in Later Stone Age and Denver Growth Study juveniles......135

Appendix D. Femoral joint dimension prediction equations and scatter plots for Later Stone Age

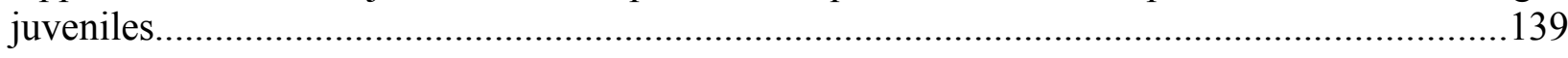

Appendix E. Body size standardized skeletal growth profiles for Later Stone Age juveniles....141 


\section{Chapter 1}

\section{Introduction}

Variation in skeletal morphology has long been a topic of interest among physical anthropologists as signal of hominin taxonomy, resulting in a rich database documenting a range of skeletal anatomy across recent human populations and our fossil ancestors. The eco-geographic clines observed for human skeletal morphology reflect the adaptive potential of bone tissue in producing phenotypic variation in body size, proportions, and robusticity. While the genetic mechanisms that mediate the potential for plasticity in bone tissue are not well understood, some of the environmental correlates of skeletal morphology are well-documented, particularly the effect of biomechanical loading in determining bone form. That skeletal morphology reflects habitual loading regimes, is a principle that has been explored by anthropologists interested in reconstructing patterns of physical activity among past populations. The last three decades have seen the development of a literature in which both the theory and method underlying the study of postcranial robusticity as a tool for understanding the subsistence and mobility behaviours of prehistoric groups, has become well established (Holt, 2003; Lovejoy, 1975; Ruff \& Hayes, 1983a, 1983b; Van Gerven et al., 1985).

Robusticity may be defined as the "strengthening or structural buttressing of a skeletal element through the addition of bone tissue" (Ruff et al., 1993). This definition 
eludes to the homeostatic process through which cortical bone is maintained, involving tissue-building cells (osteoblasts), but which also includes a role for tissue-removing cells (osteoclasts) in order to manage the competing demands of bone strength and overall skeletal mass toward optimal mechanical function. While cortical bone is subject to this homeostatic process throughout the lifespan, adulthood is principally a period of maintenance, and eventually of senescence. As has been demonstrated by clinical researchers interested in bone quality, the crucial window of opportunity for building cortical bone mass ends with puberty. Participation in weight-bearing exercise during childhood has been identified as a modifiable risk-factor in the prevention of osteoporosis, as physical activity is key to maximizing skeletal reserves of cortical bone. The variability described in postcranial robusticity among prehistoric adult skeletal samples, can therefore, be expected to reflect patterns of physical activity in the children of those populations.

Postcranial robusticity, quantified by measures of strength in long bone diaphyses, is the product of the apposition of cortical tissue throughout growth. While there is a history of studying linear growth in prehistoric populations, few studies have examined appositional growth from an ontogenetic perspective (Cowgill \& Hager, 2005). Differences in robusticity have been noted across hominin taxa (Lovejoy, 1975; Trinkaus \& Churchill, 1999; Trinkaus \& Ruff, 1989, 1999); and, from the rare specimens of juvenile fossil remains, unique patterns in the trajectories and timing of bone mass acquisition have been suggested (Ruff et al., 1994; Thompson \& Nelson, 2000). The 
description of growth trajectories and the timing of developmental events in various populations is crucial to understanding the factors that underlie the patterns of variability observed in adult samples (Pearson \& Lieberman, 2004). Recent work by Cowgill (Cowgill, 2006, 2007, 2008, In press; Cowgill \& Hager, 2005, 2007) has set out to address this gap through ontogenetic analyses of postcranial robusticity in a wide range of palaeolithic and modern human juvenile samples. This research has been effective in demonstrating that population differences in postcranial robusticity are established at a very early age.

Analyses of growth that use samples of fossil hominins are limited by the rare and fragmentary nature of the remains, which presents challenges to sample size and, importantly, to the validity of estimates of age at death. Skeletal growth analyses are dependent on the confidence with which age at death can be estimated for each individual in the sample, which is challenging in the absence of teeth associated with the postcranial remains and when patterns of dental development differ from the modern human reference samples on which dental age estimation methods are based. Analyses of juvenile fossil specimens have, nevertheless, made an important contribution to elucidating patterns of postcranial variability among hominin taxa, and have established an interpretive framework for understanding the evolutionary history of human mobility and subsistence behaviours.

The research presented herein explores appositional growth in a prehistoric 
Holocene population, represented by a sample of well-preserved remains that span the course of development from infancy to late adolescence. The quality of the preservation provides for confidence in the estimates of age at death that underpin the analyses, as the majority of individuals are represented by associated dental and postcranial remains, from single burial contexts. The completeness of the skeletons studied allows for the measurement of robusticity at multiple sites within each individual, allowing for comparisons both between and within long bone elements. This level of preservation is required for assessing the localized or systemic nature of individual robusticity levels and for evaluating laterality within the skeleton with respect to activities using the upper limb.

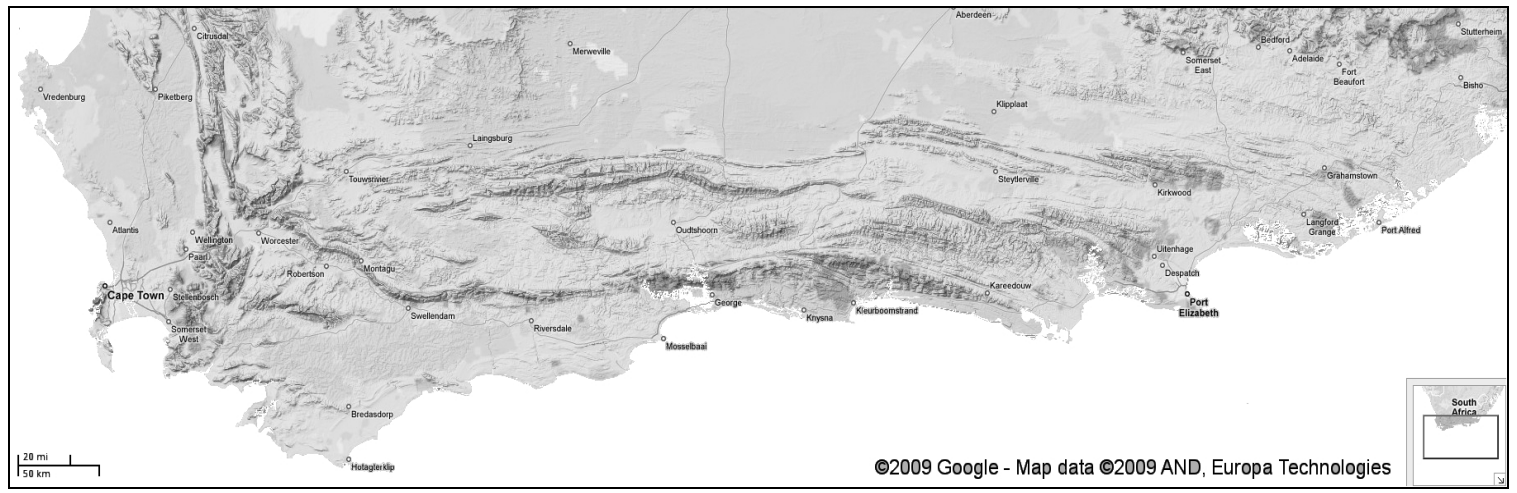

Figure 1.1. The Cape region of southernmost Africa.

Skeletons included in the sample were recovered from Later Stone Age sites located throughout the area pictured.

The study population are hunter-gatherers that occupied the coastal and adjacent mountain landscape of the Cape region of southernmost Africa (Figure 1.1). The time period represented by the sample skeletons is determined through uncalibrated radiocarbon dates of approximately 8000 to 800 years before present. Hunting and gathering is the mode by which members of the human lineage have subsisted throughout 
our evolutionary history, albeit in various forms and intensities depending on the particular characteristics of the ecological niche occupied. The well-preserved skeletal remains of the juvenile hunter-gatherers selected for study, therefore, comprise an appropriate sample for contributing to our understanding of the range of variability in postcranial robusticity in response to the habitual loading regimes associated with subsistence activities.

The juvenile skeletons selected for study are but a subset of collections that include many adult skeletons from the population of Later Stone Age hunter-gatherers that occupied southernmost Africa, and were in many cases buried in association with these adult skeletons. Existing research describes sexually dimorphic patterns of postcranial robusticity in the adult population, and offers interpretations of the behaviours reflected in the skeletal morphology in the context of archaeological evidence for tool use behaviours and subsistence strategies employed by Later Stone Age hunter-gatherers (Stock, 2002; Stock \& Pfeiffer, 2001, 2004). The research presented here builds on this scholarship by exploring the ontogeny that underlies the adult phenotype. Analysis of the timing and tempo of appositional growth in relation to the known adult "end-point" for development may add resolution to the problem of understanding the relative roles of systemic and environmental factors in determining adult morphology. It is important to note that an analysis of growth based on a cross-sectional sample - wherein the developmental "trajectory" is comprised of single observations of different children representing a range of ages, in contrast to a longitudinal analysis where subsequent 
observations on the same group of children are recorded - is limited; rates of growth cannot be determined from cross-sectional samples, which has the effect of muting evidence for sudden changes in tempo. Cross-sectional growth profiles provide a generalized picture of the overall growth pattern.

In addition to describing the nature of the appositional growth process that produces the postcranial morphology observed in Later Stone Age hunter-gatherers, this research examines the developmental timing of changes in magnitude of postcranial strength in the context of hypotheses about the mobility and subsistence behaviours in which children of various ages might typically engage. Relative strength of the lower limb relative to the upper limb within individuals of different ages can be suggestive of changes in locomotory behaviour throughout development, while asymmetry and side dominance in the upper limbs may reflect habitual manipulative behaviours associated with tool use. This research seeks to test hypotheses about specific physical activity patterns in Later Stone Age hunter-gatherer children that are generated through the literature describing the role of the child in the subsistence activities of various ethnographically-known hunter-gatherer societies. In particular, the ethnographic accounts of the !Kung (who are known today as the Ju/'hoansi) generated by the Harvard Kalahari Study offer a particularly unique opportunity to generate culturally-informed hypotheses about day-to-day life for Later Stone Age children. The cultural and biological evidence linking the Later Stone Age population of the southernmost Cape with the $\mathrm{Ju}^{\prime} /$ hoansi of the twentieth-century Kalahari, is invoked in this research as an 
interpretive framework.

The analytical approach for addressing the research goals introduced above was organized around a set of questions:

1) What is the age- and site-specific patterning of postcranial strength properties in Later Stone Age juveniles?

a. Do different strength properties (areas, second moments of area, polar second moments of area, biomechanical shape) follow the same age-specific patterns?

b. Do different strength properties follow the same site-specific (between limbs, between sides, and within limbs) patterns throughout development?

c. What is the variance in bone strength properties among individuals in infancy, childhood, and adolescence?

2) Can the patterns of postcranial robusticity that have been described in Later Stone Age adults be identified in the juveniles of this population?

a. Is there evidence for bilateral asymmetry in the upper limb?

b. Is there evidence for the expected pattern of relative arm-to-leg strength?

3) How do developing body proportions relate to developing bone strength?

a. Do bone length, joint dimensions, and limb proportions co-vary with strength measures? 
b. What are the implications for size-standardization of bone strength measures in an ontogenetic sample?

These research questions form the basis for the organization of the results chapter, while the themes of mobility and subsistence activities are used to focus the discussion and results. Before presenting the results, additional background information informing this research is presented in chapter 2 , and a detailed account of the methods employed in collecting the data is outlined in chapter 3 . 


\section{Chapter 2}

\section{Background}

\subsection{Bone functional adaptation and the role of growth}

Bone serves a number of roles as a tissue, organ, and structural element of the human body, and its morphology reflects the competing demands of its various functions. There is a long history of research directed at understanding the interplay of factors contributing to the formation and adaptive capacity of bone, particularly with respect to remodelling of cortical bone. Several factors have been identified as determinants of bone morphology including genotype, hormone levels, nutrition, disease status and, physical activity level. Mechanical influences on bone are well understood and have been popularized as “Wolff's Law" (Wolff, 1892, 1986); this term, however, has been set aside in light of significant refinements to our knowledge of bone response, and confusion around the terminology (Bertram \& Swartz, 1991; Pearson \& Lieberman, 2004).

"Bone functional adaptation" (Ruff et al., 2006) is an alternative term for the principle that bone structure is influenced by mechanical loading of skeletal elements. This principle has become an established theoretical basis for interpreting postcranial morphological variation in archaeological and fossilized skeletons as an indicator of behaviour in past populations (Larsen, 1997; Ruff, 2008). In order to make appropriate 
inferences about physical activity levels during life based on the morphology preserved in postcranial remains it is important to recognize that localized mechanical demands on bone are balanced with systemic influences on the skeleton. Interpretations of variation in postcranial robusticity, especially between populations or taxa, must acknowledge the effects of genes, diet, disease, hormones (and other potential factors) in determining bone form.

The mechanical influence on bone can be modelled as a simple feedback loop (Rubin \& Lanyon, 1984). Long bone shafts respond to strain by increasing the volume of cortical bone in the plane of deformation. Strain may be generated by increased body size (including additional muscle mass) or by increased levels of activity, which in turn increase mechanical load to the bone. An optimal level of strain in the face of increased loading is achieved through the deposition of bone tissue; in an environment of reduced strain, bone is resorbed in order to return to the optimal level. The basic idea of a feedback loop for the mechanical response of bone to habitual loading has been refined by various researchers. Perhaps the most well known of these variants is Frost's "Mechanostat" hypothesis $(1987 ; 2003)$ which likens cortical bone remodelling to a process under the control of a thermostat. Key to this hypothesis is the idea that the range for optimal strain is determined by genes. Levels of strain outside of the geneticallydetermined thresholds stimulate deposition or resorption through bone remodelling in order to return bone to an optimum customary strain level. 
An alternative feedback model proposed by Carter and Beaupré (2001), provides for more complexity by including a developmental component to the bone response. In this scenario, bone remodelling is a function of two complementary factors whose weight of influence shifts throughout development. The "biological" factor is the intrinsic determinant of bone form, and is most significant during the earliest part of development. By mid-childhood, the importance of the biological factor gives way to the "mechanobiological" factor, which encompasses the response of bone to the mechanical environment. Both intrinsic and extrinsic factors are necessary for normal bone formation, but the relative effect changes over the course of development. Control of bone remodelling in this model is, again, determined by a range bounded by thresholds termed the "lazy zone" and which contains a set-point termed the "attractor stimulus". Like with the mechanostat model, strain levels outside of the optimal customary levels will stimulate bone deposition or resorption, but this "output parameter" may be influenced by systemic factors. Carter and Beaupré acknowledge a role for metabolic components such as nutritional and disease status in shifting the set point and optimal range.

During growth, the biomechanical demands on bone are especially dynamic. As the long bones of the postcranial skeleton gain length, improving their effectiveness as moment arms, growing muscle mass also acts to generate increasing levels of strain. The process of skeletal growth is a challenge of continually constraining the deformation of 
bone to optimal levels under the threat of structural failure (Rauch \& Schöenau, 2001).

Puberty is a period of unique osteogenic potential because of the particular hormonal changes that characterize this stage of the lifespan. The skeletal response to mechanical loading seems to be influenced by elevated levels of growth hormone and the sex hormones (MacKelvie et al., 2002). Although the mechanisms for the endocrine effects on bone modelling are not well understood, observations from both human and animal studies demonstrate the age effects of exercise on bone mass. Levels of asymmetry in the strength and area measurements of the humeri of racquet sport players are significantly higher among individuals who began training before puberty (over controls) when compared with individuals who began training after puberty (over controls) (Haapasalo et al., 1998; Kontulainen et al., 2002).

Both linear and appositional bone measurements can be examined in the context of growth dynamics. Linear growth refers primarily to the length of the long bones, and serves as an analog to height in living children. Appositional growth refers to the thickness of diaphyses, which are comprised of compact, or cortical bone. Appositional growth therefore offers a gross perspective on the quality of growing bone. The amount of cortical bone is a function of the net effects of the apposition and resorption of tissue. Cortical bone is gained and lost throughout the lifespan in a predictable manner (Garn, 1970). Bone tissue is laid down or resorbed at both the outer (periosteal) and inner (endosteal) surfaces of the diaphysis independently. Periosteal apposition tracks linear 
growth and continues into adulthood with a tendency for men to put on more bone at the periosteal surface than women. During childhood, bone is resorbed at the endosteal surface at a rate that does not exceed periosteal apposition. The result is an increased diaphyseal diameter, with no difference between boys and girls. During puberty, endosteal resorption ceases and apposition begins at the endosteal surface.

Studies of living children have demonstrated that linear bone growth is preserved at the expense of cortical bone (Adams \& Berridge, 1969; Barr, Prader, \& Shmerlin, 1972; Garn, Guzman, \& Wagner, 1969; Himes et al., 1976). Reduced cortical thickness has been observed under conditions of severe protein calorie malnutrition; Garn (1969) proposed that decreased cortical thickness is due to an actual loss of bone rather than a failure to gain. Following a period of acute protein calorie malnutrition, children who gained bone length had lost cortical thickness, supporting the idea that cortical-dumping occurs. Measures of appositional growth in archaeological samples may therefore be more reflective of insults to the health of the group than measures of linear growth alone can be. Indeed, some researchers have employed this approach (Armelagos et al., 1972; Dupras, 2003; Hummert, 1983; Mays, 1995, 1999; Van Gerven et al., 1985).

Studies of living children have also demonstrated the importance of physical activity as a factor influencing bone growth. Bone mineral content is positively correlated with regular physical activity during childhood, and has also been shown to have a significant influence on bone mineral accrual during the adolescent growth spurt 
(Malina, 2002). Weight-bearing activity confers the greatest bone mineral accrual, although regular physical activity does not influence bone growth in length. The osteogenic influence of activity is generally specific to the skeletal sites at which the mechanical strains occur. Apposition of bone at the periosteal surface confers the greatest biomechanical advantage because the effect of increasing bone size moves the mass of the bone further from the central axis, enabling the bone to bear more weight. Therefore, changes in bone structure can affect bone strength without significant changes in bone density. Loading bone during growth has the effect of increasing periosteal apposition in childhood, and increasing endosteal apposition during and post-puberty (Bass, 2003). Researchers have begun to examine the sex-specific influences of physical activity on cortical bone growth. Preliminary studies by Shona L. Bass and colleagues $(1999,2002)$ have shown that while loading before puberty may result in periosteal apposition in both boys and girls, loading after puberty may result in a sex-dependent pattern. Males appear to continue to gain tissue at both surfaces under loading, while females only show endosteal apposition. The implication is that females only have the pre-pubertal period to increase bone mass, while males have a longer window of opportunity.

The quality of bone, both as an element of the skeleton and as a tissue, is operationally defined in terms of fracture strength because of the clinical relevance of fractures in aging populations. Frost (1999) identifies four factors that determine bone's fracture strength: bone mass, architecture, the material properties of the tissue, and 
microdamage in a bony element. The material properties of bone do not vary significantly with age, sex, bony element, or species, except under pathological conditions. Therefore material properties do not impact bone quality in the context of growth. Similarly, Frost's fourth factor, microdamage, has little bearing on the growth patterns of bone because it is associated with aging. Architecture, which refers to "the size and shape of the whole bone and the distribution of spongiosa and compacta within" (1999) is however, an important consideration when assessing bone strength. New techniques for assessing bone strength in living individuals employ peripheral quantitative computed tomography (pQCT) to quantify a bone strength index, which accounts for both bone mass and architectural factors. The emphasis on whole bone architecture in assessing bone quality in a clinical setting means that an increasingly standardized and tested set of techniques have been established for assessing the biomechanical properties of archaeological bone using cross-sectional morphology (Lieberman et al., 2004; Ruff, 1991; Trinkaus \& Ruff, 1999). These techniques may have application in describing the changes in whole bone architecture throughout growth, with the aim of developing a picture of what constitutes the normal pattern for a given element. Cortical bone is sensitive to insults to health. Therefore, overall bone architecture and measures of diaphyseal thickness may impart information about the quality of child growth.

Animal studies also provide evidence for the age-related effects of mechanical 
loading on bone. A study of rats subjected to treadmill running showed that cortical bone thickness was increased in a group of young rats, but no effects on cortical bone thickness were observed in a group of older rats (Steinberg \& Trueta, 1981). Lieberman and colleagues' (2003) study of treadmill exercised sheep grouped as juveniles, sub-adults, and mature adults demonstrated that cortical bone modelling at the periosteal surface of diaphyses is greater among younger individuals.

\subsection{Variability in human postcranial robusticity}

The study of postcranial robusticity among adult fossil hominins has illustrated evolutionary trends in bone functional adaptation and provided insight into the habitual behaviours of human ancestors. Ruff and colleagues' $(1993 ; 2005)$ analyses of fossil and archaeological Homo specimens dated to between two million and 8000 years ago illustrates that relative bone strength has decreased by about one third during the history of our genus, and that half of this reduction has occurred during the Holocene (Ruff, 2008). This progressive gracilization can be attributed to significant reductions in the physical demands made on the skeleton that accompany technological and cultural change.

Developmental trajectories in bone strength properties of the femur, tibia and humerus were compared among Late Pleistocene Neandertals and modern humans, and seven Holocene archaeological populations (Cowgill, 2008). Population differences are 
apparent during growth, often appearing before one year of age. Because these differences are consistent within the upper and lower limbs, the source of variation in strength is hypothesized to be systemic in nature; likely a function of genetic and nutritional differences between groups.

Late Pleistocene juveniles fall within the range of variation of Holocene juveniles. Among Late Pleistocene adults, there are few differences in levels of robusticity in the lower limb, once bone strength measures are controlled for body mass (Holliday, 2002; Trinkaus \& Ruff, 1999). Late Pleistocene adults do, however, have different diaphyseal shapes in the lower limb (Churchill et al., 2000; Holt, 2003, 1999). The upper limbs of Late Pleistocene adults show a temporal trend in robusticity with levels declining between Neanderthals and Middle Upper Palaeolithic modern humans, and a return to higher levels of robusticity in the Late Upper Palaeolithic. This temporal trend in the upper limb was not detected in Cowgill's (Cowgill, 2008; In Press) relatively small sample of Late Palaeolithic juveniles. Cowgill's finding of few significant differences between juvenile early and more recent populations stands in contrast to the evidence for a steady decline in levels of robusticity among adults from early Homo to the Holocene (Ruff et al., 1993). Recent evidence suggests that the decline in bone strength observed in adult Holocene populations may not be universal, with some Holocene groups maintaining high levels of robusticity (Shackelford, 2005, 2007). Cowgill's research among juveniles also demonstrates that the wide range of variation in levels of bone strength among Holocene 
groups overlaps with levels observed in the Late Pleistocene. There are many examples of individual immature Late Pleistocene individuals with levels of robusticity that fall well within the range of variability reported for Holocene juveniles. Comparisons of postcranial robusticity among recent and Late Pleistocene juveniles are limited by the ability to estimate age among Late Pleistocene juveniles with confidence. Fossilized remains are often fragmentary and incomplete, and rarely associated with dental remains from the same individual. Age estimates for Late Pleistocene individuals may be based on long bone or dental development standards for modern children, in lieu of an alternative. This methodological limitation likely contributes in some part to the inconsistencies in reports of relative levels of robusticity between Late Pleistocene and Holocene individuals of "similar age".

\subsection{Later Stone Age hunter-gatherers}

The foraging environment of the South African Cape was successfully exploited since the emergence of Homo sapiens. Coastal Middle Stone Age sites like Klasies River Mouth (Singer \& Wymer, 1982), which is dated to $c a .90,000$ to 120,000 B.P., show that people exploited shellfish and other marine resources, as well as terrestrial foods. Indeed, Klasies River Mouth has been called the oldest known sea food 'restaurant' (Deacon \& Deacon 1999). Other Middle Stone Age sites are located far from the coast; the tool kit and faunal remains all suggest exploitation of a wide range of foods through active hunting and family foraging groups. During the Last Glacial Maximum, the southern 
African interior became relatively cold and dry, and populations were attracted toward the southern coast. Cycles of aridity have affected human habitation of the interior, but rock shelters in the Cape Fold Mountains and river mouths appear to have remained attractive locales (Deacon \& Deacon, 1999).

During the Holocene (the last 10,000 years), the distribution of archaeological sites indicates that foraging groups most commonly exploited the coastal regions and the variable but generally plentiful resources of the fynbos biotic province, with fewer people exploiting the interior grasslands. The fynbos, literally "fine bush," is comprised of herbaceous plants like ericas, proteas and restionacae, with a low representation of grasses (Meadows \& Sugden, 1993). Within the fynbos and throughout southern Africa, various native plants produce highly nutritious underground storage organs in the form of roots, bulbs, corms and rhizomes. Southern Africa is known for its floral diversity, said to include some 30,000 species of flowering plants, accounting for almost ten percent of the world's higher plants (van Wyk \& Gericke, 2000). These plants provide habitats for a wide range of animal life, from small rodents and tortoises through to large antelopes and elephants. There is a richly productive marine ecosystem, thanks to the mixing of the icy Benguela current of the South Atlantic and the warm Agulhas current of the southwest Indian Ocean. Marine protein, including fish, marine mammals and mollusks were generally abundant, especially along rocky shorelines. In modern times archaeological sites are often first identified through the presence of very large shell middens. 
There are multiple lines of evidence supporting an ancestor-descendant relationship between Later Stone Age foragers and historically known Khoe-San peoples of the Kalahari region. If habitual behaviours affect the skeleton, hypotheses can test whether ethnographically documented Khoe-San behaviours extended back in time and into the richer environment of the Cape. Archaeological sites from $c a$. 40,000 B.P. to the historic era are categorized as Later Stone Age. The initial Holocene stone tool assemblage known as Oakhurst (or a variant known as Albany), was replaced by the Wilton assemblage in the first half of the Holocene (Deacon \& Deacon, 1999). All Later Stone Age assemblages include more bone, shell and ostrich eggshell artifacts than the earlier Middle Stone Age complexes. Variation in the proportions of small scrapers, bladelets and adzes are some of the regional distinctions upon which archaeologists base reconstructions of foraging adaptations during this time period (Mitchell, 2002). At about 2000 years B.P., some pastoralism (evidence of sheep herding) appears in the region, as well as the production of pottery.

The Holocene Later Stone Age human skeletons come from rock shelters, excavated with variable levels of expertise, and from many chance discoveries of skeletons buried in sand dunes. The pattern of adult body size is short stature, gracile frame (Sealy \& Pfeiffer, 2000; Pfeiffer \& Sealy, 2006), and lean physique. The latter is deduced from the ubiquitous presence of squatting facets and evidence of knee hyperflexion in adult skeletal remains (Dewar \& Pfeiffer, 2004). It is around the end of the 
Pleistocene that evidence of light-draw bows (and presumably poison-tipped arrows) is first found (Parkington, 1998; Wadley, 1998). Measures of upper arm bone strength and asymmetry indicate a gender-based division of labour in which women's activities probably focused on work with digging sticks and grinding, while men hunted. Elements of the tool kit show homogeneity through the region, though there are also local patterns. Analysis of upper arm asymmetry suggests that men in the southern (mountain forest) and western (fynbos) regions appear to have favoured spears, and light-draw bows, respectively (Pfeiffer \& Stock, 2002; Stock \& Pfeiffer, 2004). This same research indicated symmetry in the upper arm strength of women throughout the Cape. This may reflect their regular use of digging sticks. As George Silberbauer writes about the G/wi:

"Women and girls forage each day for food plants within an $8 \mathrm{~km}$ radius of the campsite. A root, tuber or bulb is dug out with a pointed digging stick, which is used to break up the sand before scraping it out by hand. This method is slow but efficient, for the equipment is light, easily portable, and made of materials that are readily available and simply converted to this purpose. Furthermore, there is no call for sudden exertion: rather, there is a slow, steady expenditure of energy, which suits the small, lightly muscled women....The work is not strenuous, but its lack of rhythm and the frequent changes of position, the slow, meandering searching, and the carrying of the growing weight of the day's find make it very tiring." (Silberbauer, 1981:199200)

A long and lively debate since the 1970s has scrutinized whether Holocene foragers of the Cape exploited regions seasonally or whether they stayed within one locale for a prolonged period (Parkington, 1972; Parkington, 2001; Sealy, 1995; Sealy, 1997; Sealy \& van der Merwe, 1988). Stable isotope research on human skeletal tissue suggests 
that groups frequently delimited their ranges to either terrestrial or coastal zones. Indeed, recent research comparing the stable isotope values of neighbouring communities along the southern coast indicates the maintenance of territories between $c a .4500$ and 2000 B.P., such that one group had access to a rich seal rookery while their neighbours did not (Sealy, 2006). On the other hand, research from the Eastern Cape coast suggests that inland people would visit the shoreline and leave shell middens behind (Binneman, 2004).

While the full range of regional food resources were probably not exploited by any one group, proteins and carbohydrates were available in variable proportions to all communities. There was seasonality in the availability, palatability, and nutritional value of the plant resources, which would have required strategic planning. Controlled burning may have been used to influence seed release and new growth (Deacon, 1976). The labour involved in collecting shellfish meant that the economic return ratio was only profitable during neap tides (Binneman, 2004). While shellfish collection does not require particular skill or strength, and is often the domain of women and children (Meehan, 1982), large amounts of shellfish can be eaten for only limited periods (Noli \& Avery, 1988). There has been debate about the food safety of a diet heavily based on shellfish (Noli \& Avery, 1988), but archaeological evidence suggests that heavy reliance on low trophic level marine protein was common among some Later Stone Age populations (Mitchell, 2002). 
Capture of terrestrial animals was achieved through trapping, as well as hunting. Tortoise bones and carapace sections are common in middens, and could be procured by children. Fats were probably rare and highly valued. This may help to explain both the high status of large antelopes like the kudu and eland in rock art and ethnography, and the apparent competition along the South Coast for access to juvenile seals. In a stable isotope $\left(\delta^{13} \mathrm{C}\right.$ and $\left.\delta^{15} \mathrm{~N}\right)$ study of 127 adults, using femur length as a proxy for stature, the source of dietary protein (terrestrial versus marine) did not explain a significant amount of stature variance, although a positive correlation that was found with femoral head diameter suggests slightly greater body mass may be linked to more reliance on high trophic level marine protein (Pfeiffer \& Sealy, 2006). The amount of food, rather than the type of protein available, was more likely to significantly affect growth.

One ethnographically documented practice of modern era Khoe-San foragers that is particularly relevant to the study of child growth is the practice of breastfeeding until a child is well past one year of age. It could be reasoned that this practice may be unique to the Kalahari, if the sparse food base of the Kalahari would put a younger weanling at risk. To explore the possibility of prolonged breastfeeding in a richer environment among Khoe-San ancestors, the stable nitrogen $\left(\delta^{15} \mathrm{~N}\right)$ and carbon $\left(\delta^{13} \mathrm{C}\right)$ isotope ratios were measured from bone collagen of 35 infants and juveniles from a large southern coast site, Matjes River Rock Shelter (Clayton et al., 2006). The isotopic values from thirty adults from the site are tightly clustered, so the trophic effect of breast milk is apparent. 
Children at Matjes River Rock Shelter show consistently heightened trophic levels for at least the first eighteen months after birth, with diminishing and increasingly variable levels thereafter. This is consistent with patterns reported for historic era Kalahari foraging communities of prolonged on-demand breastfeeding, cessation of breast feeding by about four years (Konner, 2005) and birth spacing of approximately three years (Howell, 1979; Lee, 1979; Schapera, 1930; Shostak, 1981). Hence, breast milk provided the dominant source of protein to forager infants, even when those foragers lived in an environment with abundant food resources.

\subsection{Childhood physical activity in the ethnographic record}

Ethnographic accounts serve as a useful basis for interpreting behaviour in prehistoric children. The Harvard Kalahari study is a particularly important source of information because of the cultural links between historically-known Khoesan groups and

Later Stone Age people, e.g., with respect to attitudes toward childhood and the sex-based division of labour. The significant differences in the ecological environments occupied by Khoesan and Later Stone Age populations may, however, be significant insofar as terrain influences mobility patterns.

As part of a multidisciplinary study of the people who were then usually called the !Kung Bushmen of Botswana (now called the Ju/'hoansi), Nancy Howell collected crosssectional information on the weights and statures of 165 children, virtually a complete 
survey of the permanent juvenile residents of the Dobe area in 1967 to 1969 (1979; 2000). She recorded a mean birth weight of $3.08 \mathrm{~kg}$ (S.D. 0.458), in ten newborns, with just one infant falling below the World Health Organization low birth weight criterion of $2500 \mathrm{~g}$. She documented heights and weights cross-sectionally of children to twenty years of age. At the same time, Patricia Draper collected behavioural information on children's movements, play patterns, and relationships (1976). The fact of small body size among the $J u$ /'hoansi was not lost on the researchers of the Harvard Kalahari Study. Biomedical researchers documented various maladies in Dobe children, but found no evidence for nutritional or infectious sources for the lessened magnitude of growth, relative to other populations (Truswell \& Hanson, 1976).

Draper and Howell have recently combined the information on growth and behaviour for a sample of fifty-one children who were included in both of their studies (Draper \& Howell, 2005). Comparing the sex-specific weight, stature, and body mass index information to that of the U.S. National Center for Health Statistics, the Ju/'hoan children are short and light. Their absolute weights are well below the median expectations, and their body mass indices are also below median values (although body mass values deviate to a lesser degree). They explored possible correlations between the variance within the growth data and variable behaviours, including time spent in the presence of the mother or father, or other children, time spent in physical contact with someone else, time spent in low energy activity, time spent in or near the home village. 
They did not find empirical support for an influence on growth coming from kinship, family composition or children's behaviours. They conclude that area kin groups did not have differential access to resources, such that some children would be better off than others. They conclude, "the multiple cross linkages created by bi-laterality, bi-local residence practises, name relationships, trading relationships, and the pervasive rule on sharing were apparently holding at the time of the study" (2005:280). Given the existence of various behavioural linkages between the Later Stone Age people and the ethnographically documented historic communities of the Kalahari, these conclusions can be incorporated as plausible assumptions behind hypotheses about the growth of Later Stone Age children. 


\section{Chapter 3}

\section{Materials and Methods}

\subsection{The sample under study}

This research examines growth among Later Stone Age hunter-gatherers of southernmost Africa. These people are known from archaeological and skeletal remains recovered from sites located throughout the coastal, and inland Cape Fold Mountain region of present-day South Africa. The 82 skeletons that are the subject of this research come from more than 30 sites throughout the region, and are from collections curated at four South African institutions (Appendix A). Many of the skeletons have been radiocarbon dated, with dates ranging from $887 \pm 27$ to $8260 \pm 720$ years before present (uncalibrated); and many were documented by the excavators with information confirming that the burial context is consistent with the mortuary custom of Later Stone Age hunter-gatherers (Deacon, 1976; Deacon \& Deacon, 1999; Mitchell, 2002). All of the skeletons included in the sample are from Later Stone Age burial contexts and/or are radiocarbon dated to the Later Stone Age period. As such, the sample is representative of the population of Later Stone Age hunter-gatherers whose cultural and biological characteristics are described above, while bearing in mind the attendant biases of archaeological human skeletal assemblages (Wood et al., 1992). 


\subsection{Estimation of age at death}

The sample was selected with the aim of representing the complete course of postnatal development in robusticity characteristics. The most immature individuals in the sample are those with skeletal development consistent with perinatal infants (Scheuer $\&$ Black, 2000); whether these remains are those of infants who were stillborn or, those who died sometime shortly after birth is impossible to determine from macroscopic observation. The individuals studied have no macroscopic skeletal indicators of significant disease or disruptions to growth, and all are represented by well-preserved and relatively complete remains. The most mature individuals in the sample are young "adults" with some degree of incomplete epiphyseal fusion, normally at the medial clavicle. In these individuals the third molars are completely erupted, but dental wear is lighter than in other fully mature adults in the collections. Because cortical bone tissue may accrue well into adulthood (Garn, 1970; Ericksen, 1976; Lazenby, 1990; Ruff et al., 1994; Bass et al., 2002), it is necessary to include young adults in this ontogenetic sample in order to observe the full range of developmental variation in postcranial robusticity.

Age at death is estimated through dental development when possible, by estimating crown and root development in all visually observable teeth (dental radiographs were not employed) using Moorrees, Fanning and Hunt's 14-stage system of formation (1963a;b). Age estimates for deciduous teeth are from Moorrees and colleagues' age of attainment charts (1963b), and estimates for permanent teeth are from Smith's (1991) adaptation for estimation of Moorrees and colleagues' attainment charts 
for the permanent dentition (1963a). Although these standards are based on the mandibular dentition, they were applied to maxillary teeth as well, in order to increase the number of individuals in the sample whose age at death could be estimated by dental development. This methodological decision reflects an assumption that the amount of error encompassed by the difference in the timing of mandibular versus maxillary development is less than the error that would result from estimating age through some other method. The relative sequence for dental development and eruption among Later Stone Age juveniles is consistent with that of modern European populations (Matzke, 2000) For each individual, the estimate of age at death is the average of scores for all teeth for which crown or root development could be observed. Fifty-one of the 82 individuals in the sample (62\%) have age at death estimates that are based on dental development.

Age at death estimation through dental development was not possible for the remaining 31 individuals. Of these, ten individuals had dental eruption information available by way of a fully erupted second or third molar, but this information was not used to estimate age at death because of the wide range of variation in the timing of eruption for these permanent teeth and the decreasing utility of later-occurring developmental events for age estimation with respect to precision (Scheuer \& Black, 2004). Had eruption-based estimates been used (Ubelaker, 1989), employing the midpoint of the range for timing of eruption for the tooth, several individuals would be given the same estimate for age at death (depending on whether the estimate was based 
on the second or third molar), but in observing all indicators of chronological age including epiphyseal fusion, it is clear that a range of developmental age is represented among these individuals.

In the ten individuals for whom only dental eruption information was available and the remaining 21 individuals who had no preserved teeth, a method was employed that attempted to seriate these individuals among the 51 individuals whose age at death was estimated by dental development. Age estimates for the 51 dentally-aged individuals were regressed on long bone lengths for these individuals, to generate equations used to predict dental age in the rest of the sample. Long bone lengths were selected for prediction of dental age rather than another developmental indicator, such as epiphyseal fusion, because of the continuous and precise nature of a length measurement versus that of a variable that relies on a qualitative score. This approach privileges the identification of the relative development of individuals within the sample over the accuracy of individual age estimates. This is both valid and desirable in this research design, which is concerned primarily with developmental patterns within a particular population, and not with making age-based comparisons to other samples. It is also important to note that dental age estimates are falsely precise because the ranges for age of attainment are not reported by Moorrees and colleagues (1963a;b). Were these ranges available, the estimate for age, which is the independent variable in most of the analyses to follow, could incorporate an error term that encompasses the range of attainment age for the developmental stage of each tooth that comprises the overall estimate of age at death. 
Equations for predicting dental age were generated through ordinary least squares regression of various long bone lengths, including and excluding epiphyses (maximal and diaphyseal lengths), with dental age as the dependent variable ("inverse calibration", Konigsberg et al., 1998) (for corresponding scatter plots, see Appendix B). The relative effectiveness of each equation for the prediction of dental age was evaluated according to the standard error of the estimate (SEE). For the 31 individuals without dental developmental information, age at death was predicted using the equation for the available preserved long bone with the lowest SEE (Table 3.1). Estimates of age at death for each individual and the source of the estimate (dental or long bone) are listed in Appendix A.

\begin{tabular}{l|l|l|l|l|l}
\hline & $\mathbf{n}$ & Intercept & Slope & $\mathbf{R}^{2}$ & SEE \\
\hline Humerus Diaphysis & 39 & -5.4068 & 0.0751 & 0.9416 & 1.0399 \\
\hline Ulna Maximum & 4 & 3.8012 & 0.0517 & 0.8256 & 1.0534 \\
\hline Tibia Diaphysis & 38 & -4.1576 & 0.0560 & 0.9489 & 1.1244 \\
\hline Femur Diaphysis & 40 & -5.0007 & 0.0514 & 0.9473 & 1.1361 \\
\hline Ulna Diaphysis & 42 & -5.9437 & 0.0915 & 0.9488 & 1.1620 \\
\hline Clavicle Maximum & 39 & -8.5396 & 0.1849 & 0.9505 & 1.1822 \\
\hline Femur Maximum & 14 & -5.5335 & 0.0503 & 0.9114 & 1.5291 \\
\hline Tibia Maximum & 11 & -4.2980 & 0.0557 & 0.8257 & 1.6181 \\
\hline Humerus Maximum & 10 & -1.5852 & 0.0609 & 0.7885 & 1.8969 \\
\hline
\end{tabular}

Table 3.1. Prediction equations for dental age from long bone lengths in Later Stone Age juveniles

The age distribution for the sample is outlined in Table 3.2 by age cohort, along with the number of individuals in each cohort whose age at death was predicted through regression. The four smallest infants have negative values for predicted age. While it is 
possible that they were pre-term given their state of epiphyseal fusion, this may in part represent a degree of under-ageing due to extrapolation beyond the range of the regression relationship. It is the relative state of development among the individuals in the sample that is most important to the validity of the following analyses, rather than the accuracy of chronological age estimates.

\begin{tabular}{|c|c|c|c|c|}
\hline $\begin{array}{c}\text { Age Cohort } \\
\text { (years) }\end{array}$ & $\begin{array}{c}\text { Cohort } \\
\text { n }\end{array}$ & \begin{tabular}{|c} 
Predicted \\
$\mathbf{n}$
\end{tabular} & $\begin{array}{c}\text { Cohort } \\
\text { Mean }\end{array}$ & $\begin{array}{c}\text { Cohort } \\
\text { SD } \\
\end{array}$ \\
\hline 0.17 & 6 & 4 & -0.23 & 0.38 \\
\hline 0.33 & 7 & 0 & 0.29 & 0.07 \\
\hline 0.50 & 4 & 0 & 0.60 & 0.10 \\
\hline 1.0 & 3 & 0 & 1.21 & 0.10 \\
\hline 2.0 & 7 & 1 & 1.98 & 0.40 \\
\hline 3.0 & 0 & 0 & - & - \\
\hline 4.0 & 3 & 0 & 4.02 & 0.42 \\
\hline 5.0 & 4 & 1 & 5.12 & 0.22 \\
\hline 6.0 & 7 & 2 & 6.01 & 0.30 \\
\hline 7.0 & 3 & 0 & 6.60 & 0.10 \\
\hline 8.0 & 5 & 3 & 7.93 & 0.38 \\
\hline 9.0 & 1 & 1 & 9.10 & - \\
\hline 10.0 & 1 & 1 & 10.07 & - \\
\hline 11.0 & 5 & 1 & 11.09 & 0.22 \\
\hline 12.0 & 4 & 3 & 12.33 & 0.10 \\
\hline 13.0 & 2 & 0 & 13.10 & 0.00 \\
\hline 14.0 & 1 & 1 & 14.32 & - \\
\hline 15.0 & 9 & 9 & 15.14 & 0.30 \\
\hline 16.0 & 7 & 3 & 15.94 & 0.15 \\
\hline 17.0 & 3 & 1 & 16.80 & 0.25 \\
\hline 18.0 & 1 & 1 & 18.27 & - \\
\hline Total & 83 & 32 & & \\
\hline
\end{tabular}

Table 3.2. Age at death estimates by age cohort in Later Stone Age juveniles 


\subsubsection{Estimation of sex}

Morphological estimation of sex in juvenile skeletal remains is unreliable because most established methods rely on the identification of skeletal characteristics that develop during puberty. Sex estimation was, however, possible for many of the older individuals included in the sample. While some sub-adult characteristics persist late into development, such as an open medial clavicle epiphysis, the pelvis may be sufficiently developed to estimate sex with confidence. This was the case for 22 of the 82 individuals in the sample (Table 3.3), all of whom have an estimated age at death of at least 13 years (Appendix A). All sex estimates are based on the standard Phenice traits (Buikstra \& Ubelaker, 1994) of the pelvis (Pfeiffer, personal communication). It is important to note that there may be a bias toward the identification of female individuals in adolescent and young adult skeletons. The morphology of the sub-adult pelvis lacks the characteristics of the adult female, that is, an undeveloped female pelvis bears more resemblance to the adult male form. Therefore, a sub-adult skeleton with female characteristics can be more confidently identified as female than an ambiguous sub-adult pelvis, which is more difficult to differentiate as either indeterminate (absent of sex characteristics) or male. Though more female than male adolescents were identified in the sample, the sex

distribution in the identified subset of the sample is not significantly different $\left(X^{2}=0.727\right.$, $p=0.394)$ from that expected by chance alone (i.e., in the absence of a taphonomic or cultural explanation for a sex sampling bias). 


\begin{tabular}{l|c}
\hline Sex & $\mathbf{n}$ \\
\hline Indeterminate & 60 \\
\hline Female & 13 \\
\hline Male & 9 \\
\hline Total & 82 \\
\hline
\end{tabular}

Table 3.3. Estimated sex distribution for Later Stone Age juveniles

\subsection{Osteometric variables}

The quantification of robusticity required measurement of the postcranial skeleton with respect to both the linear dimensions of the long bones and the cross-sectional geometry of cortical tissue about the diaphyses.

\subsubsection{Linear skeletal dimensions}

The linear dimensions of the long bones of each individual were measured for two purposes: first, to quantify body size and shape; and secondly, in order to locate positions along the proximal-distal axis of diaphyses where cross-sectional measurements would be taken. The long bones studied are the clavicle, humerus, ulna, femur, and tibia; the selection of these elements is based on the objective of quantifying each of the biomechanically relevant limb segments in both the upper and lower limbs. To simplify the dataset, only one of the two long bones comprising the distal limb segments was selected for study - ulna and tibia - based on the availability of comparable published data. Both the upper and lower limbs were studied bilaterally in order to test assumptions about the base levels of asymmetry prior to the initiation of activities that demand significant weight-bearing in the limbs. 
The main challenge encountered in measuring limb bone lengths was the variability in fusion of the long bone epiphyses throughout the sample. The main linear measurements in this study are the maximum lengths of the diaphyses alone, without epiphyses. In the oldest individuals, however, where both proximal and distal epiphyses were fused, reported lengths are maximum lengths including epiphyses. For some adolescents, epiphyseal fusion was in progress at the time of death, in some cases such that only one of the proximal or distal epiphysis was fused. Where possible, both maximal and diaphyseal lengths were estimated for these limbs. Preservation of the epiphyses is very good in this sample, which allowed for measurement of both epiphyseal and metaphyseal dimensions. Maximum lengths including epiphyses were not, however, reconstructed for individuals with unfused epiphyses because such lengths would be shorter than living limb lengths due to the soft tissue located between the diaphysis and epiphysis, and the practical constraints of performing such measurements of reconstructed elements with accuracy. The maximum breadth of the femoral head epiphysis, and the medio-lateral breadth of the distal femoral metaphysis were measured for estimation of body mass.

\subsubsection{Cross-sectional geometry}

Robusticity has been defined as the "strengthening or structural buttressing of a skeletal element through the addition of bone tissue" (Ruff et al., 1993:22). In the simplest of terms, robusticity refers to the relative thickness of bone or strength of build: a variable quality readily-observed through gross observation of human skeletons from a 
range of populations, in both the cranial and postcranial skeleton. With respect to postcranial robusticity, early analyses focused on cortical thicknesses, expressed as an index of cortical to total diaphyseal width. This approach has since been extended to include analysis of the mechanical competency of long bones, which is determined by the the distribution of cortical bone tissue about the diaphysis in cross-section. Trabecular bone tissue is also organized in response to mechanical demands upon the skeleton, but the scope of this research is limited to the study of postcranial robusticity in terms of the distribution of cortical bone throughout the diaphyses of long bones.

Cross-sections of the diaphysis can be reconstructed at any position along the proximal-distal axis of the long bones (Ruff \& Hayes, 1983), however, those that have become standardized in the anthropological biomechanics literature are the locations that were selected for this research. A total of 16 locations were selected, as outlined in Table 3.4 and Figure 3.1. 


\begin{tabular}{l|c|l|l}
\hline Element & Side & Position & Abbreviation \\
\hline Clavicle & Left & Mid-shaft (50\%) & CLM \\
\hline & Right & Mid-shaft (50\%) & CLM \\
\hline Humerus & Left & Mid-shaft (50\%) & HLM \\
\hline & Left & Distal (35\%) & HLD \\
\hline & Right & Mid-shaft (50\%) & HRM \\
\hline & Right & Distal (35\%) & HRD \\
\hline Ulna & Left & Mid-shaft (50\%) & ULM \\
\hline & Right & Mid-shaft (50\%) & URM \\
\hline Femur & Left & Subtrochanteric (80\%) & FLST \\
\hline & Left & Mid-shaft (50\%) & FLM \\
\hline & Right & Subtrochanteric (80\%) & FRST \\
\hline & Right & Mid-shaft (50\%) & FRM \\
\hline Tibia & Left & Nutrient Foramen & TLNF \\
\hline & Left & Mid-shaft (50\%) & TLM \\
\hline & Right & Nutrient Foramen & TRNF \\
\hline & Right & Mid-shaft (50\%) & TRM \\
\hline
\end{tabular}

Table 3.4. Diaphyseal cross-section locations studied

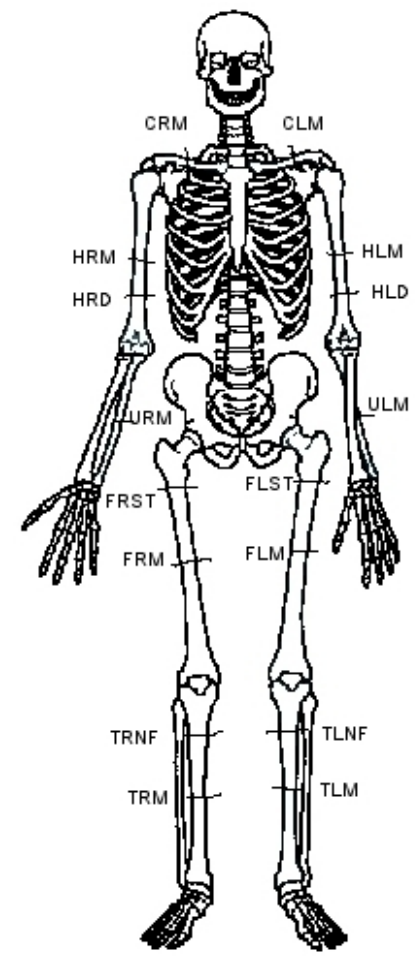

Figure 3.1. Skeletal locations of crosssections studied

Analysis of the cross-sectional geometry of long bones provides quantification of

three types of properties: areas, second moments of areas, and polar second moments of areas. Area measurements include estimates of the total diaphyseal area, the area of the medullary cavity, and area of the cortical tissue. Of these, cortical area is most relevant with respect to biomechanics as is it reflects the strength (or rigidity) of the long bone in resisting force transmitted along the proximal-distal axis through the centroid of the section (Ruff \& Hayes, 1983). Total area is important insofar as tissue furthest away from the centroid has the most effect on bending and torsional rigidity (Bertram \& Swartz, 1991; Wainwright, 1982). Second moments of area reflect the bending strength 
of a long bone relative to a given axis, and are dependent on the distribution of cortical bone about the centroid. Bending strength in the antero-posterior plane about the mediolateral axis is conventionally termed $\mathrm{I}_{\mathrm{x}}$, and bending strength in the medio-lateral plane about the antero-posterior axis is designated Iy. Bending strength can, theoretically, be measured relative to any axis but the use of bi-planar radiographs allows for calculation of second moments relative to the selected radiographic planes only. The polar second moment of area is the sum of any two perpendicular second moments, and reflects the torsional strength of a long bone. The biomechanical properties that are the main dependent variables in this research are summarized in Table 3.5.

\begin{tabular}{l|c|l|c}
\hline Cross-Sectional Property & Abbreviation & Biomechanical Significance & $\begin{array}{c}\text { Unit of } \\
\text { measurement }\end{array}$ \\
\hline Total area & TA & Influences second moments of area & $\mathrm{mm}^{2}$ \\
\hline Cortical area & CA & Axial strength (tensile and compressive) & $\mathrm{mm}^{2}$ \\
\hline Medullary area & MA & Area of the medullary cavity & $\mathrm{mm}^{2}$ \\
\hline $\begin{array}{l}\text { Second moment of area } \\
\text { about x-axis }\end{array}$ & $\mathrm{I}_{\mathrm{x}}$ & $\begin{array}{l}\text { Bending strength } \\
\text { in antero-posterior plane }\end{array}$ & $\mathrm{mm}^{4}$ \\
\hline $\begin{array}{l}\text { Second moment of area } \\
\text { about y-axis }\end{array}$ & $\mathrm{I}_{\mathrm{y}}$ & $\begin{array}{l}\text { Bending strength } \\
\text { in medio-lateral plane }\end{array}$ & $\mathrm{mm}^{4}$ \\
\hline $\begin{array}{l}\text { Maximum } \\
\text { second moment of area }\end{array}$ & $\mathrm{I}_{\mathrm{max}}$ & Maximum bending strength & $\mathrm{mm}^{4}$ \\
\hline $\begin{array}{l}\text { Minimum } \\
\text { second moment of area }\end{array}$ & $\mathrm{I}_{\mathrm{min}}$ & Minimum bending strength & $\mathrm{mm}^{4}$ \\
\hline $\begin{array}{l}\text { Polar } \\
\text { second moment of area }\end{array}$ & $\mathrm{J}$ & Torsional strength & $\mathrm{mm}^{4}$ \\
\hline $\begin{array}{l}\text { Theta } \\
\text { (Angle between } \mathrm{I}_{\mathrm{x}} \text { and } \mathrm{I}_{\mathrm{max}}\end{array}$ & $\theta$ & $\begin{array}{l}\text { Orientation of plane of } \\
\text { maximum bending strength }\end{array}$ & $\mathrm{degrees}^{4}$ \\
\hline
\end{tabular}

Table 3.5. Biomechanical properties studied 
When long bones are modelled as hollow beams, their biomechanical properties can be calculated using standard engineering formulae from the dimensions of the cortical bone in cross-section (Huiskes, 1982). In lieu of destructive sectioning, radiography (or computed tomography) is used to create images from which the dimensions of the medullary cavity can be measured. The primary technique used in this research involved six measurements taken from two sets of radiographs for each individual: one in antero-posterior view to generate measurements of the medio-lateral subperiosteal breadth (ML), and the breadths of the medial (m) and lateral (l) cortices; and a second radiograph in medio-lateral view for measurements of the antero-posterior subperiosteal breadth (AP), and the breadths of the anterior (a) and posterior (p) cortices. Elements of similar size and radio-opacity were arranged on film cassettes in standard positions with the aid of modelling clay (anatomical for the AP view, and rotated $90^{\circ}$ for the ML view). A radio-opaque wire was placed at the level of the nutrient foramen of the tibia so that measurements could be taken from the radiographs at this point. The other measurement positions were located on the radiographs by calculating the appropriate percentage of maximum (or diaphyseal) length $(35 \%, 50 \%, 80 \%$ from distal end). Radiographs were back-lit using a light box and cortical dimensions were measured with Mitutoyo callipers to the nearest tenth of a millimetre. Combining the six measurements creates a reconstruction that models the diaphyseal cross-section as an ellipse (representing the medullary cavity) within an ellipse (the subperiosteal surface) as illustrated in Figure 3.2. Because each cortex is individually measured, the medullary 
ellipse is not necessarily concentric with the ellipse representing the subperiosteal surface. This “asymmetrical” (Biknevicius \& Ruff, 1992; Runestad et al., 1993), or “eccentric ellipse” model (Ohman, 1993; Stock, 2002) accounts for any asymmetrical distribution of cortical tissue about the diaphysis captured by the bi-planar radiographs.

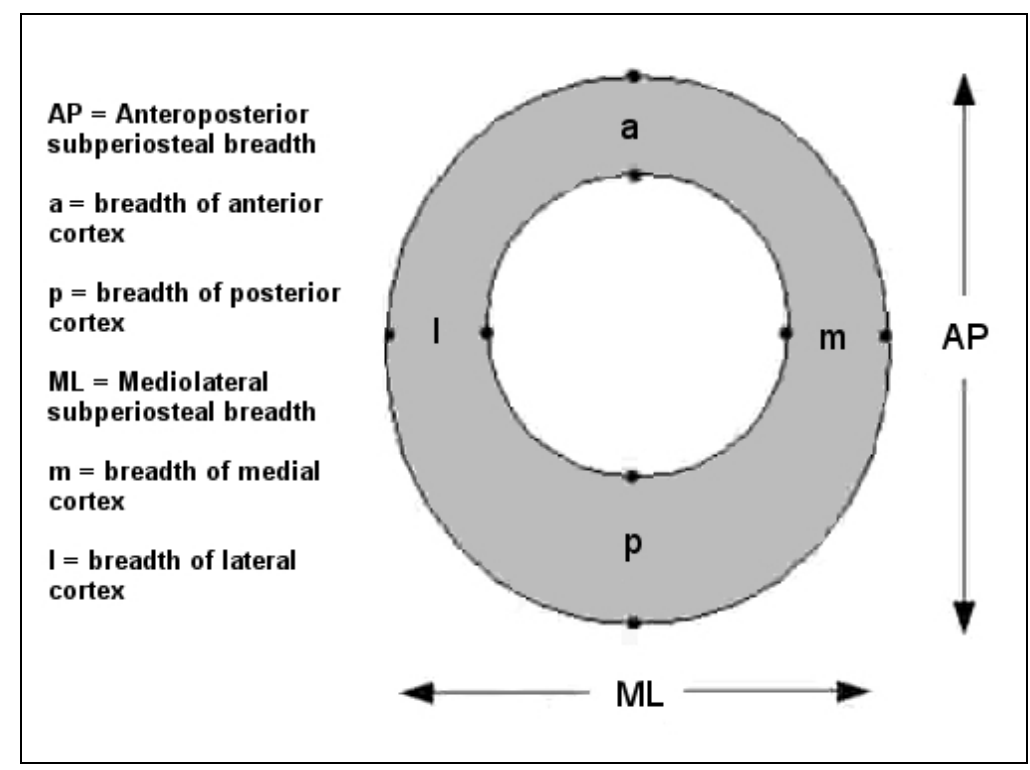

Figure 3.2. Eccentric ellipse model of a diaphyseal cross-section

The six measurements from the radiographs were used to calculate biomechanical properties following formulae provided by Ohman (1993; after Milgrom, 1989) (Table 3.6). The formulae for areas are adapted from standard engineering formulae for properties of a hollow beam. Calculation of second moments of area from an eccentric ellipse model employs the parallel axis theorem, whereby the position of the centroid $\left(\mathrm{X}_{\mathrm{o}}, \mathrm{Y}_{\mathrm{o}}\right)$ is determined from the eccentric placement of the centroid of the medullary ellipse within the subperiosteal ellipse (Milgrom, 1989). 


\begin{tabular}{|c|c|}
\hline $\begin{array}{l}\text { Cross- } \\
\text { Sectional } \\
\text { Property }\end{array}$ & Formulae from Eccentric Ellipse Model \\
\hline Total Area & $T A=\pi \frac{(A P \cdot M L)}{4}$ \\
\hline $\begin{array}{l}\text { Medullary } \\
\text { Area }\end{array}$ & $M A=\pi \frac{(A P-a-p) \cdot(M L-m-l)}{4}$ \\
\hline Cortical Area & $C A=T A-M A$ \\
\hline $\begin{array}{l}\text { Centroid } \\
\text { Position, ML } \\
\text { axis }\end{array}$ & $X_{o}=\frac{(l-m) \cdot(A P-a-p) \cdot(M L-l-m)}{(2[M L \cdot(a+p)+(l+m) \cdot(A P-a-p)])}$ \\
\hline $\begin{array}{l}\text { Centroid } \\
\text { Position, AP } \\
\text { axis }\end{array}$ & $Y_{o}=\frac{(a-p) \cdot(A P-a-p) \cdot(M L-l-m)}{(2[M L \cdot(a+p)+(l+m) \cdot(A P-a-p)])}$ \\
\hline $\begin{array}{l}\text { Second } \\
\text { Moment of } \\
\text { Area, ML } \\
\text { plane }\end{array}$ & $I_{x}=T A\left(\frac{M L^{2}}{16}+X_{o}^{2}\right)-M A \frac{\left[16 X_{o}\left(X_{o}+l-m\right)+5\left(l^{2}+m^{2}\right)-2 l(3 m+M L)+M L(M L-2 m)\right]}{16}$ \\
\hline $\begin{array}{l}\text { Second } \\
\text { Moment of } \\
\text { Area, AP } \\
\text { plane }\end{array}$ & $I_{y}=T A\left(\frac{A P^{2}}{16}+Y_{o}^{2}\right)-M A \frac{\left[16 Y_{o}\left(Y_{o}+a-p\right)+5\left(a^{2}+p^{2}\right)-2 a(3 p+A P)+A P(A P-2 p)\right]}{16}$ \\
\hline $\begin{array}{l}\text { Polar Second } \\
\text { Moment of } \\
\text { Area }\end{array}$ & $J=I_{x}+I_{y}$ \\
\hline
\end{tabular}

Table 3.6. Formulae for calculating biomechanical properties of a hollow beam After Ohman (1993); see Figure 3.2 for definitions of coefficients.

A second method for determining cross-sectional geometry was used on a subset of section locations: cross-sections of the mid-shaft femur and humerus, bilaterally, were reconstructed using the "latex cast method" (terminology, Stock, 2002). This method is used to reconstruct cross-sections by combining measurements of the cortical dimensions from bi-planar radiographs with a cast of the subperiosteal surface (Trinkaus \& Ruff, 1989). The maximum length of the long bone was measured and the mid-shaft position was marked; then, plasticine was placed around the diaphysis distal to the mark, serving 
as a base for the molding material. The molding material was applied and once hardened, the cast was marked on its anterior surface and removed from the diaphysis after cutting along its medial side. By marking and cutting the casts in this way, the cast could be oriented subsequently to anatomical position. The selected casting medium (Exaflex vinyl polysiloxane, GC America) is a clinical-grade material that holds the shape of the diaphysis' circumference, does not shrink or expand, and provides detailed resolution of the subperiosteal surface.

Images of the latex casts were created with a flatbed scanner to a resolution of 600 dots-per-inch in anatomical position, along with a scale. The inclusion of a scale in the image allowed for calibration of the image dimensions (in pixels per millimetre). Using imaging software (ImageJ, Rasband) and a digital pen and tablet (Wacom CTE-440), the image of the cast was magnified and the subperiosteal boundary was traced manually. To reconstruct the medullary boundary, the dimensions of the cortices from the bi-planar radiographs were used to locate the anterior, posterior, medial and lateral margins of the medullary cavity, and an ellipse was traced manually to join the four points. The software was used to automatically fill the region between the subperiosteal and endosteal boundaries, representing the cortical area. The result is an image comprised of a true representation of the subperiosteal circumference with an estimated medullary circumference, that can be analysed with the software to calculate cross-sectional geometric properties (Figure 3.3). 


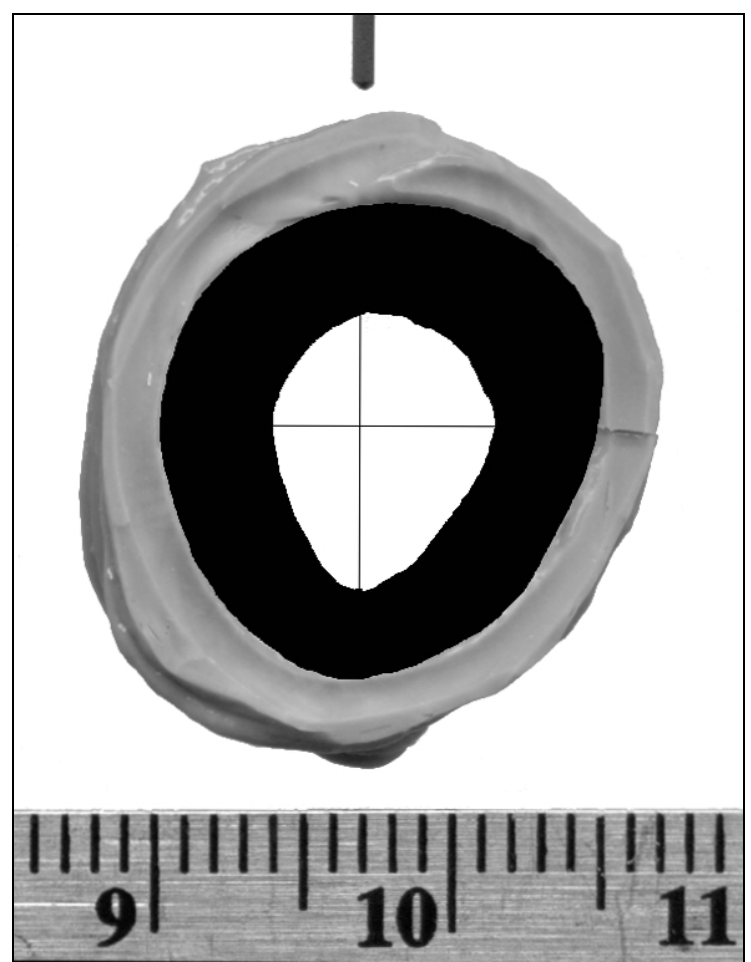

Figure 3.3. A cross-sectional reconstruction using the latex cast method

An advantage of the latex cast method is that by reconstructing the diaphyseal cross-section with the true subperiosteal boundary, the principal axes of bending rigidity - the second moments of area, Imax and Imin - can be identified in addition to Ix and Iy. Areas, second moments of area, and theta $(\theta)$, the angle between Ix and Imax (measured counter-clockwise), were calculated from the image using a macro written for ImageJ by Christopher Ruff (MomentMacroJ).

\subsection{Estimation of body mass}

Size standardization of biomechanical properties required estimation of body mass for each individual. To estimate body mass in each Later Stone Age juvenile, agespecific regression formulae generated by Ruff (2007) from the Denver Growth Study 
(Maresh 1943; 1955; 1970) were used. The Denver Growth Study is a longitudinal anthropometric study of twentieth-century North American children conducted between 1927 and 1967. In that study, subjects were radiographed and weighed every two months from birth to six months of age, and at six-month intervals thereafter until late adolescence; a sub-sample of ten females and ten males with nearly complete longitudinal datasets were selected by Ruff. His analysis demonstrated that the relationship between skeletal dimensions and body mass changes continuously throughout growth, necessitating age-specific regression formulae for the prediction of body mass from joint dimensions (2007).

Reduced major axis (RMA) regression formulae for predicting body mass (in kilograms) from natural log transformed measurements of the breadth of the distal femoral metaphysis (medio-lateral) and femoral head epiphysis (supero-inferior) are reproduced in Table 3.7. Ruff's RMA, rather than OLS, regression formulae were selected for predicting body mass in the Later Stone Age juveniles because this alternative regression technique is recommended for populations outside the size range of the Denver sample (see Appendix C for Denver and Later Stone Age femoral dimensions). Because of the timing of the fusion of epiphyses and the visibility of landmarks in the Denver Study radiographs, the distal femoral metaphysis could be measured (and therefore, formulae available) up to age seven, and the femoral head epiphysis after age six. In several age classes ( 2 months, 6 months, 14-16 years) the equations fail to reach statistical significance and are not recommended for use (Ruff, 
2007; personal communication).

\begin{tabular}{|c|c|c|c|c|}
\hline \multirow{2}{*}{$\frac{\text { Age Class }}{(\text { years })}$} & \multicolumn{2}{|c|}{ Femoral Distal Metaphysis } & \multicolumn{2}{|c|}{ Femoral Head Epiphysis } \\
\hline & Slope & Intercept & Slope & Intercept \\
\hline 0.17 & n.s. & n.s. & - & - \\
\hline 0.33 & $1.649 \dagger$ & $-3.7^{\dagger}$ & - & - \\
\hline 0.50 & n.s. & n.s. & - & - \\
\hline 1 & 1.157 & -1.9 & - & - \\
\hline 2 & 1.235 & -2.2 & - & - \\
\hline 3 & 1.503 & -3.2 & - & - \\
\hline 4 & 1.595 & -3.5 & - & - \\
\hline 5 & 1.600 & -3.5 & - & - \\
\hline 6 & 1.581 & -3.3 & - & - \\
\hline 7 & 1.544 & -3.1 & 0.908 & 0.1 \\
\hline 8 & - & - & 1.123 & -0.6 \\
\hline 9 & - & - & 1.781 & -2.8 \\
\hline 10 & - & - & 2.107 & -4.0 \\
\hline 11 & - & - & 2.397 & -5.0 \\
\hline 12 & - & - & 2.344 & -4.9 \\
\hline 13 & - & - & 2.684 & -6.1 \\
\hline 14 & - & - & n.s. & n.s. \\
\hline 15 & - & - & n.s. & n.s. \\
\hline 16 & - & - & n.s. & n.s. \\
\hline 17 & - & - & 2.186 & -4.2 \\
\hline
\end{tabular}

Table 3.7. Regression statistics for predicting body mass from femoral joint dimensions in juveniles based on Denver Growth Study data

From Ruff (2007: Table A1) and $\dagger=$ Ruff, personal communication; n.s.=equation not significant for the age class; body mass in kilograms, joint dimensions in millimetres, natural log transformed.

In order to predict body mass in Later Stone Age juveniles of the age classes for which there are no reliable formulae, alternative techniques were employed. To predict body mass in the 2-month cohort, extrapolation was employed using the equation generated from regression (OLS) of predicted body mass on distal metaphyseal breadth in the Later Stone Age 4-month-olds (Figure 3.4a). Interpolation was employed for 
prediction of body mass in the 14-, 15-, and 16-year olds: body mass was regressed (OLS) on femoral head epiphyseal breadth in Later Stone Age individuals from the 13and 17-year-old age classes, and the resultant equation used to predict body mass in 14 to 16-year olds (Figure 3.4b). To work around the lack of an equation for the 6-month cohort, body mass was predicted using the equation for the 4-month cohort.

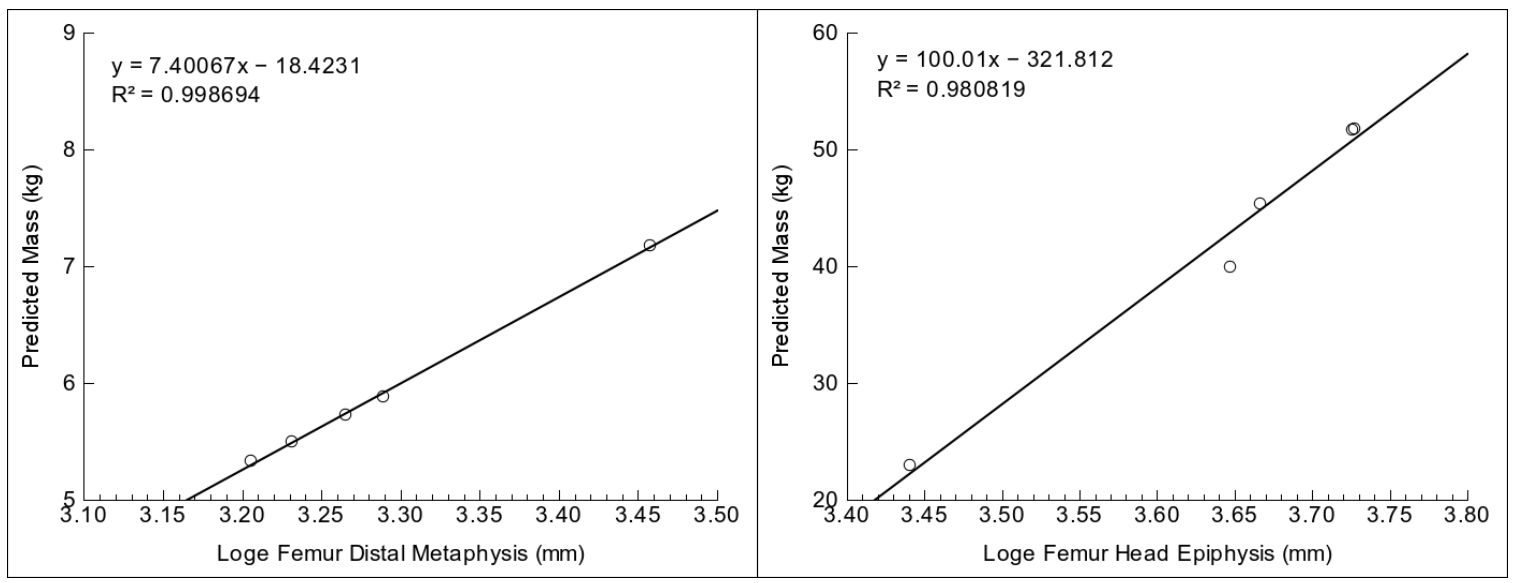

Figure 3.4. Equations for the prediction of body mass in Later Stone Age juveniles in the absence of Denver Growth Study formulae

a) Equation used to predict body mass in Later Stone Age juveniles aged 3 months and under; b) Equation used to predict body mass in Later Stone Age juveniles aged 13.5-16.5 years.

A number of Later Stone Age juveniles lacked a preserved femoral joint dimension necessary for the prediction of body mass: 9 of the 41 individuals $(22 \%)$ for whom body mass was predicted using the femoral distal metaphysis were missing, and 11 of the 41 individuals $(26 \%)$ for whom mass was predicted using the breadth of the femoral head epiphysis. To estimate these missing data, prediction equations were generated by regressing femur distal metaphysis breadth and femur head epiphysis breadth on various long bone dimensions in Later Stone Age individuals for whom these 
measurements were preserved. The relative predictive value of the various formulae were assessed using the SEE (Table 3.8), and the best available equation was used to predict the femoral joint dimension necessary (femur distal metaphysis for individuals aged under 6.5 years, and femur head epiphysis for individuals aged over 6.5 years).

Scatter plots for the femoral joint dimension prediction equations can be found in Appendix D. Body mass estimates for Later Stone Age juveniles are summarized in Table 3.9 by age cohort, and individual predicted body masses are listed in Appendix A.

\begin{tabular}{l|l|c|c|c|c|c|c}
\hline Dependent Variable & Independent Variable & $\mathbf{n}$ & $\mathbf{R}^{2}$ & Slope & Intercept & SEE & Predicted n \\
\hline $\begin{array}{l}\text { Femur Distal } \\
\text { Metaphysis }\end{array}$ & $\begin{array}{l}\text { Tibia Proximal } \\
\text { Metaphysis }\end{array}$ & 38 & 0.988 & 1.114 & 2.482 & 1.321 & 4 \\
\hline & Clavicle Maximum & 32 & 0.953 & 0.642 & 6.594 & 2.583 & 5 \\
\hline $\begin{array}{l}\text { Femur Head } \\
\text { Epiphysis }\end{array}$ & $\begin{array}{l}\text { Tibia Proximal } \\
\text { Epiphysis }\end{array}$ & 23 & 0.961 & 0.624 & 1.928 & 0.960 & 3 \\
\hline & Ulna Maximum & 13 & 0.747 & 0.134 & 6.645 & 1.693 & 3 \\
\hline & $\begin{array}{l}\text { Tibia Proximal } \\
\text { Metaphysis }\end{array}$ & 9 & 0.895 & 0.903 & 10.776 & 2.010 & 2 \\
\hline & Ulna Diaphysis & 13 & 0.880 & 0.147 & 5.560 & 2.045 & 2 \\
\hline & Clavicle Maximum & 24 & 0.701 & 0.208 & 10.805 & 2.706 & 1 \\
\hline
\end{tabular}

Table 3.8. Femur joint dimension prediction equations for Later Stone Age juveniles 
The predictions for body mass are within the expected range relative to available comparable data. Ten perinatal infants studied as part of the Harvard Kalahari Project (Howell, 2000) had a mean weight of 3.08 kilograms $(S D=0.458$; males $n=5$, mean=3.30, $\mathrm{SD}=0.366$; females $\mathrm{n}=5$, mean $=2.85, \mathrm{SD}=0.459$ ). The infants in the youngest cohort in the Later Stone Age sample have a mean estimated body mass of 4.19 kilograms $(\mathrm{SD}=0.37)$, but this includes infants up to three months of estimated age. The other relevant published body mass data are estimates of body mass in Later Stone Age adults from the southern Cape (Sealy \& Pfeiffer, 2000), who, based on body mass estimates derived from measures of femoral head breadth and bi-iliac breadth, are estimated to weigh 42.8 kilograms among males $(n=8, S D=6.6)$ and 38.3 kilograms among females $(n=5, S D=4.4)$. The estimates obtained here based on femoral head breadth alone are slightly greater, with a mean body mass estimate of 49.6 kilograms $(\mathrm{SD}=3.70)$ in three individuals with estimated ages at death between 16.5 and 17.5 years (Table 3.9). The body mass estimates predicted based on the equations generated from the Denver Growth Study are within the range of other relevant studies, and are therefore suitable for use in standardizing the cross-sectional properties in this research. 


\begin{tabular}{|c|c|c|c|}
\hline $\begin{array}{c}\text { Age Cohort } \\
\text { (years) }\end{array}$ & $\mathbf{n}$ & Mean Mass (kg) & SD \\
\hline 0.17 & 6 & 4.19 & 0.37 \\
\hline 0.33 & 7 & 4.52 & 1.21 \\
\hline 0.50 & 4 & 5.99 & 1.46 \\
\hline 1.0 & 3 & 7.62 & 0.76 \\
\hline 2.0 & 7 & 8.78 & 1.78 \\
\hline 3.0 & 0 & - & - \\
\hline 4.0 & 3 & 11.76 & 0.96 \\
\hline 5.0 & 4 & 12.00 & 2.13 \\
\hline 6.0 & 7 & 15.86 & 1.53 \\
\hline 7.0 & 3 & 18.79 & 0.78 \\
\hline 8.0 & 5 & 23.96 & 1.74 \\
\hline 9.0 & 1 & 20.09 & - \\
\hline 10.0 & 1 & 25.19 & - \\
\hline 11.0 & 5 & 28.84 & 6.60 \\
\hline 12.0 & 4 & 32.24 & 9.31 \\
\hline 13.0 & 2 & 31.46 & 12.02 \\
\hline 14.0 & 1 & 31.65 & - \\
\hline 15.0 & 9 & 36.95 & 2.83 \\
\hline 16.0 & 7 & 43.36 & 5.99 \\
\hline 17.0 & 3 & 49.60 & 3.70 \\
\hline 18.0 & 1 & 45.46 & - \\
\hline
\end{tabular}

Table 3.9. Predicted body mass in Later Stone Age juveniles by age cohort

\subsection{Statistical methods}

The objective of this research is to evaluate the ontogeny of postcranial robusticity in one archaeological skeletal population. Developmental patterns in cross-sectional properties of long bones were analysed using skeletal growth profiles (Hoppa \& FitzGerald, 1999), which are scatter-plots of various osteometric dependent variables 
against the independent variable of estimated age at death. Locally-weighted regression (LOESS) curves were fit to the scatter-plots to evaluate age-dependent changes in crosssectional properties. LOESS regression provides a weighted fit to subsets of the independent variable (Cleveland \& Devlin, 1988) which has the advantage of modelling age ranges independent of one another while providing a description of the overall developmental pattern. This is an appropriate approach in this research because in making an initial evaluation of the growth pattern in the absence of a standard model selected $a$ priori (e.g., linear, exponential, or some growth function), any unique patterns within particular age ranges of the cross-sectional developmental trajectory can be explored. LOESS curves were calculated based on $50 \%$ of the data points to provide a moderate level of smoothing, using the Epanechnikov weighting method which gives less weight to extreme points.

After describing the relationship between cross-sectional properties and estimated age from the LOESS curve, regression testing was performed to find the curve of best fit among eight standard models (linear, inverse, cubic, quadratic, compound, S, growth, and exponential). The curve of best fit was identified using the r-squared value. Unstandardized residuals from the best-fitting regression equation were then plotted against the independent variable (estimated age) to evaluate ontogenetic variation in cross-sectional properties. Residuals were plotted by sex estimate (indeterminate in the younger individuals in the sample) to evaluate the role of sex in bone functional adaptation. It is important to note that the models included in the curve-fitting analysis 
are not necessarily plausible biological models for growth. The goal was to select a model that best accounted for the age-related variation in the dependent variable in order to minimize residuals.

In order to explore the functional relationship between biomechanical characteristics of long bones and body size throughout growth, step-wise linear regression was employed to model the relationship between cross-sectional properties and size-related independent variables. This analysis was performed to partition the roles of body mass and lever arm length (diaphyseal length) as determinants of element strength, as they relate to the size-standardization of cross-sectional properties.

Cross-sectional properties of the femoral and humeral mid-shaft derived from the latex cast method, were standardized to means of the adult Later Stone Age population as percentage growth achieved by age. Raw strength properties (not standardized to body mass) for male and female southern African adults (femur $n=53$, female/male $=30 / 23$; humerus $n=49$, female/male/unknown=27/21/1) were made available (Stock, personal communication) and combined-sex means were calculated. The adults included are individuals with complete epiphyseal fusion of the long bones, from three of the four collections represented by the juvenile sample that forms the basis of this research. That is, Eastern Cape individuals are represented in the juvenile sample but not in the adults. This sampling difference is assumed to have no significant effect on the analysis. Juvenile cross-sectional properties were calculated as percentages of the adult sex- 
combined mean and plotted against age to evaluate the achievement of adult strength throughout the course of development in the cross-sectional sample. 


\section{Chapter 4}

\section{Results}

Cross-sectional properties, including areas and polar moments of area, are reported for sixteen diaphyseal locations (Table 3.4, Figure 3.1). Results are presented in four sections corresponding to the research questions outlined in chapter 1. Skeletal growth profiles for diaphyseal areas and torsional strength, for each cross-sectional location are presented in section 4.1 , organized by skeletal element. Upper to lower limb strength proportions, and relative strength of proximal and distal elements within limbs are explored in section 4.2. Section 4.3 presents an analysis of biomechanical shape, and side asymmetry for both the lower and upper limbs. Finally, the role of body size as a determinant of femoral and humeral robusticity is explored in section 4.4 , as well as the effect of size-standardization in an ontogenetic analysis of bone functional adaptation.

\subsection{Skeletal growth profiles for postcranial robusticity}

Total, cortical, and medullary areas, and torsional strength properties derived from formulae for the eccentric ellipse model method (EEM) (Table 3.6) are reported for the mid-shaft (50\%) femur, tibia, clavicle, humerus, and ulna. A secondary location is reported for the femur (subtrochanteric, 80\%), tibia (nutrient foramen), and humerus (distal, 35\%); both the left and right limbs were analysed, for a total of sixteen cross- 
sectional locations. The choice of postcranial elements allows for between- and withinlimb (proximal vs. distal segment) comparisons. The inclusion of two cross-sectional locations for the longest elements (femur, tibia, and humerus) allows for within-element comparisons.

Regression, with LOESS curve and additional curve-fitting, forms the basis of analysis. Analysis of growth achievement relative to mean values for Later Stone Age adults, was performed for the mid-shaft femur and humerus. Standardization relative to the adult mean was possible only for these cross-sectional locations because they were measured using the latex cast method (LCM, described in section 3.3.2) in addition to the eccentric ellipse model method. The LCM-derived values for the juveniles could be compared to unpublished adult values (Stock, personal communication) because the adult values were also derived from the LCM and are therefore comparable.

\subsubsection{Cross-sectional properties of the femur}

Total (TA), cortical (CA) and medullary area (MA) of subtrochanteric and midshaft femur increase with age in a manner that, from visual inspection of the scatter-plots (Figures 4.1 and 4.2), appears linear. The slight differences between the LOESS curves for the left and right limbs may be due to methodological error as there is no functional explanation for significant lower limb asymmetry. The differences in the slopes of the total, cortical, and medullary area trajectories reflect a thickening of the cortex relative to the total subperiosteal area throughout development. There is no evidence that diaphyses 
have ceased expanding in size by late adolescence among Later Stone Age juveniles.

There is more scatter in the dimensions of the subtrochanteric location when compared to that of the mid-shaft femur. This is likely, in part, attributable to the fact that the subtrochanteric cross-section of the diaphysis is more irregular in shape; depending on the location of the greater trochanter relative to the $80 \%$ length measurement, the cross-section may be larger if immediately distal to the greater trochanter or smaller if further distal. The subtrochanteric total areas tend to be slightly larger than mid-shaft, as expected from the anatomy. Cortical area, however, is greater relative to total area at the mid-shaft location in contrast to the subtrochanteric. It follows that the mid-shaft region of the femur imparts more axial compressive and tensile strength than the region of the shaft immediately distal to the greater trochanter.

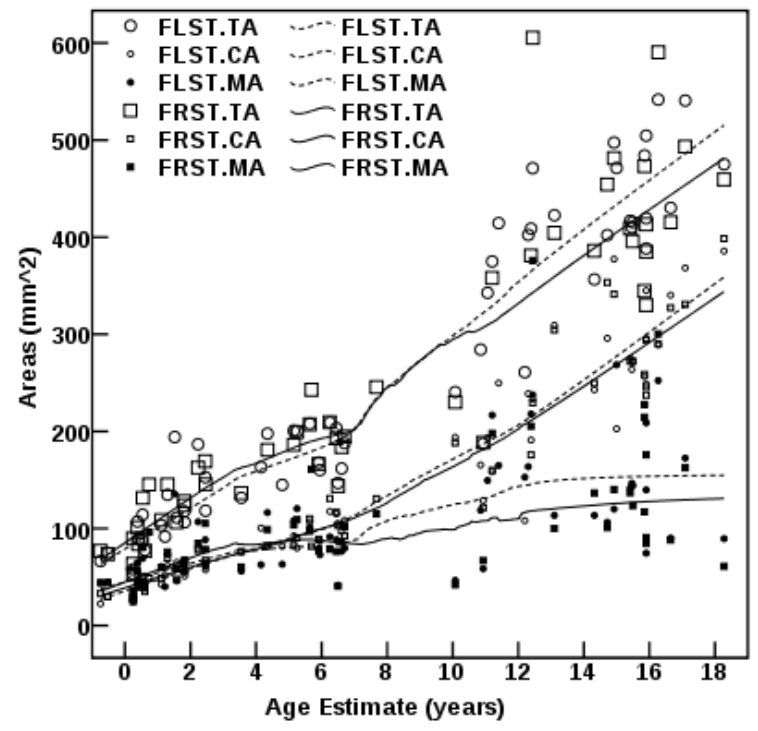

Figure 4.1. Areas (total, cortical, and medullary) of subtrochanteric $(80 \%)$ femur

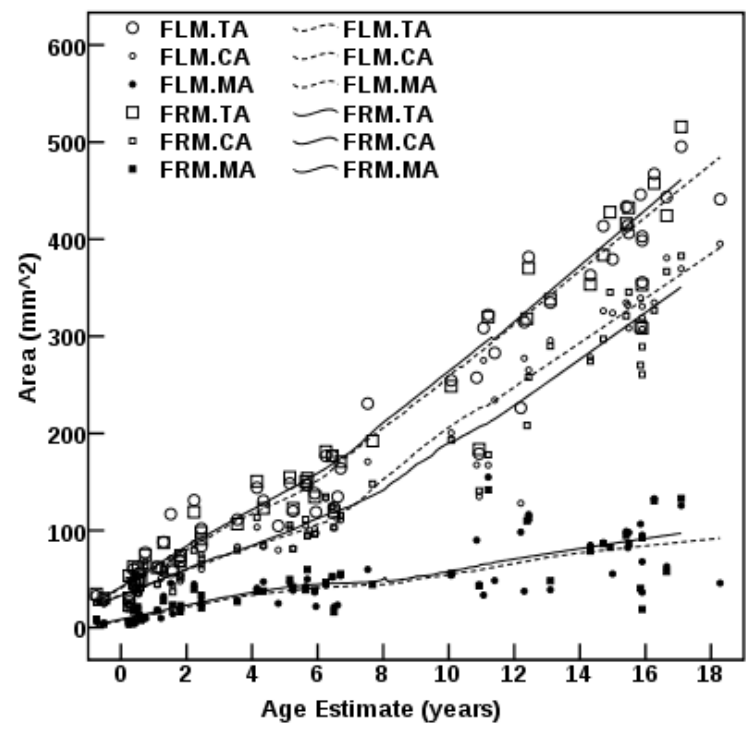

Figure 4.2. Areas (total, cortical, and medullary) of the mid-shaft $(50 \%)$ femur 
Skeletal growth profiles for torsional rigidity (polar second moment of area, or, J) show similar patterns at the subtrochanteric and mid-shaft locations in both magnitude and trajectory (Figures 4.3 and 4.4). From birth to approximately seven years of age, strength increases to about $4000 \mathrm{~mm}^{4}$, after which the rate of strength increases to values up to ten times greater by late adolescence. The difference in the rates of increase between these two developmental phases of the growth profile may be exaggerated by the paucity of individuals with estimated ages at death in late childhood (aged eight to ten years), though arguably these data indicate a functional difference in biomechanical properties by the onset of adolescence. The increase in variance in strength measures after age seven is further evidence of functional differences emerging after childhood. There is more variance in the later part of the developmental period in the subtrochanteric location than in the mid-shaft for the reasons explained above, though levels of torsional strength are similar between the proximal and distal section location. Whereas levels of axial compressive and tensile strength were greater in the mid-shaft femur relative to the subtrochanteric location, torsional forces seem to be borne equally by the proximal and mid-shaft regions of the diaphysis. 

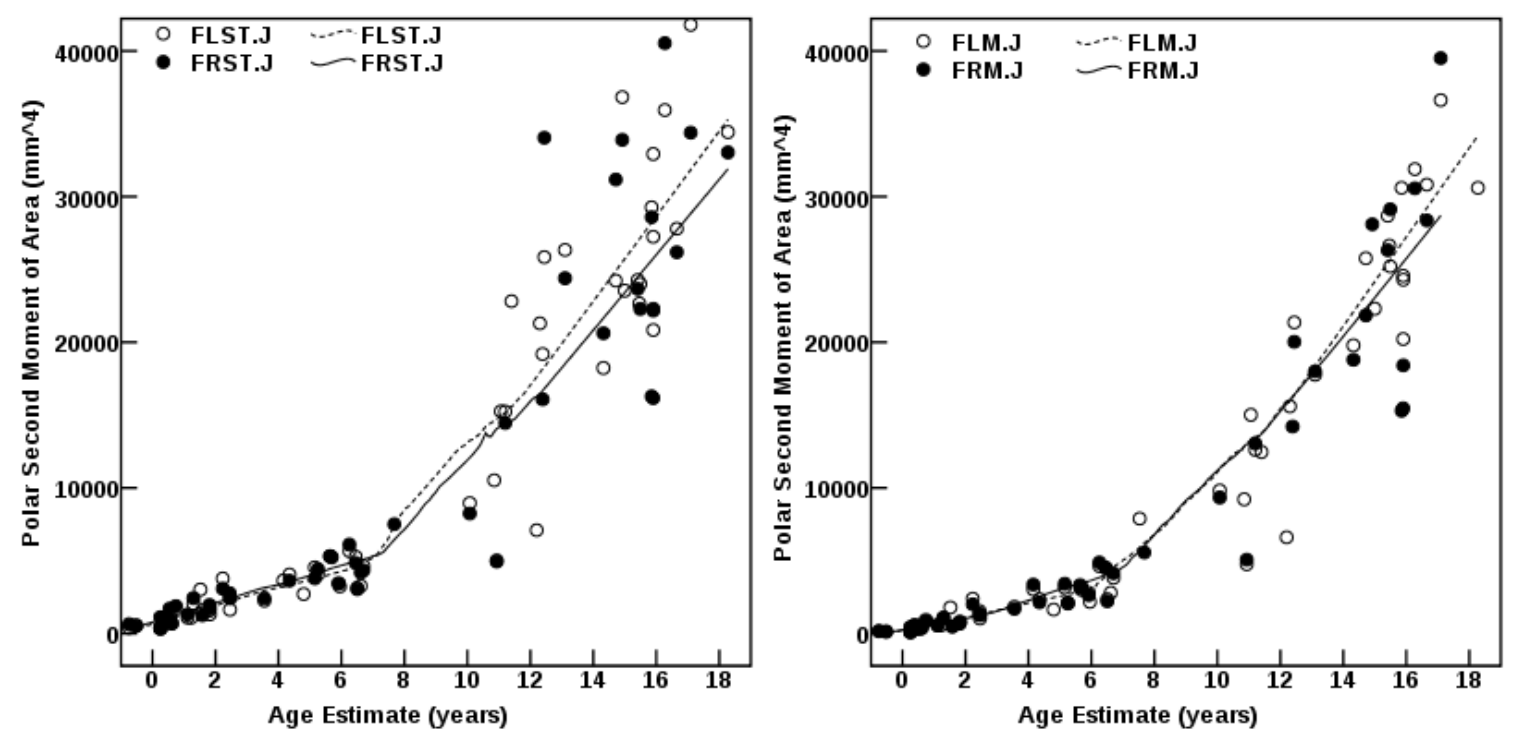

Figure 4.3. Torsional rigidity $(\mathrm{J})$ of subtrochanteric $(\mathbf{8 0} \%)$ femur

Figure 4.4. Torsional rigidity (J) of mid-shaft $\mathbf{( 5 0 \% )}$ femur

Figure 4.5 illustrates torsional rigidity in the mid-shaft left femur, expressed as a percentage of the mean for Later Stone Age adults. All juveniles aged younger than approximately 13 years have torsional strength values less than $50 \%$ of the adult mean; in other words, most femoral torsional strength is accrued post-puberty. The adult mean is not achieved until late adolescence: in just two individuals aged greater than 16 years.

The sex estimates in this plot indicate no strong sex difference in femoral strength during adolescence: while all males are nearly at or greater than the LOESS curve for the sample, there are approximately equal numbers of females above and below the curve. If males have absolutely stronger lower limbs than females in the Later Stone Age population as would be predicted given the greater muscle mass of males, this difference does not emerge until after adolescence. 

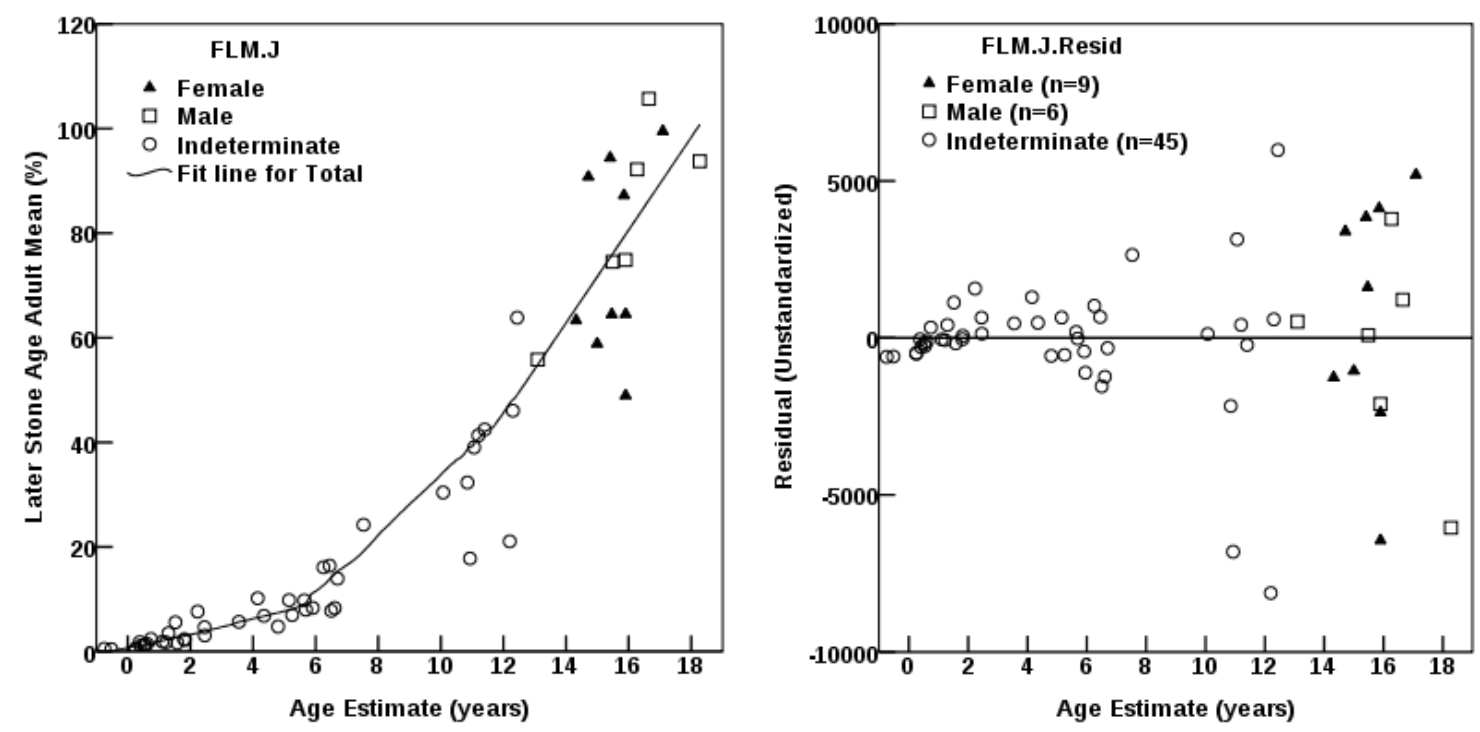

Figure 4.5. Torsional rigidity $(J)$ of the mid-shaft Figure 4.6. Unstandardized residuals for torsional $(50 \%)$ left femur standardized to the adult Later rigidity $(J)$ of mid-shaft $(50 \%)$ left femur Stone Age population mean

Each of the area and torsional rigidity skeletal growth profiles was analysed for best fit from among a number of standard regression curves as described in section 3.5 . A cubic model consistently produced high r-squared values among the models tested. Table 4.1 gives the r-squared values for each cross-sectional property in each element (left sides). In general, age explains more of the variation in lower limb cross-sectional geometry than it does in the upper limb. Medullary area values are least-well explained by age; the area scatter-plots illustrate that in adolescence, medullary area is more variable than either total or cortical area. 


\begin{tabular}{l|c|c|c|c}
\hline & \multicolumn{4}{|c}{ Cubic Model R-Squared } \\
\cline { 2 - 5 } & Total Area & Cortical Area & Medullary Area & Torsional Rigidity (J) \\
\hline Femur & 0.958 & 0.954 & 0.603 & 0.949 \\
\hline Tibia & 0.922 & 0.893 & 0.690 & 0.899 \\
\hline Clavicle & 0.900 & 0.938 & 0.281 & 0.903 \\
\hline Humerus & 0.900 & 0.929 & 0.393 & 0.883 \\
\hline Ulna & 0.891 & 0.912 & 0.215 & 0.864 \\
\hline
\end{tabular}

Table 4.1. R-squared values for regression of mid-shaft cross-sectional properties on age fit with a cubic model

Unstandardized residuals from the cubic model are used to analyze age-related variation in torsional rigidity. Figure 4.6 illustrates that for the mid-shaft location in the left femur, there is relatively little variation in torsional strength among children aged under seven years. With late childhood and continuing into adolescence, torsional strength values become highly variable within the sample. The sex estimates for individuals aged greater than 13 years illustrate that this variability cannot be attributed to a sex-based difference in strength: while adolescents are highly variable in levels of femoral torsional strength relative to children, absolute strength does not tend to be greater in either sex.

\subsubsection{Cross-sectional properties of the tibia}

Skeletal growth profiles for the biomechanical properties of the tibia follow the same patterns observed for the femur. The LOESS curves suggest that areas increase in a linear fashion with increasing age at both the nutrient foramen (Figure 4.7) and mid-shaft (Figure 4.8) locations. There is no appreciable difference in area values for the left and right elements. The differences in the slopes of the total and cortical area trajectories 
relative to that of the medullary area reflect a thickening of the tibial cortex in older juveniles. As is observed in the femur, the proximal section location has less cortical tissue relative to total area than is observed at mid-shaft; functionally, this difference reflects an adaptive response to greater axial compressive and tensile force at mid-shaft. In terms of absolute size, the nutrient foramen section location tends to be slightly larger for age than the mid-shaft tibia; this pattern is like that observed in the femur, with the proximal location being slightly larger than mid-shaft, reflecting the proximal-distal tapering in circumference of both elements. On the whole, area measurements for the femur tend to be slightly larger than the tibia at any given age throughout the developmental trajectory.

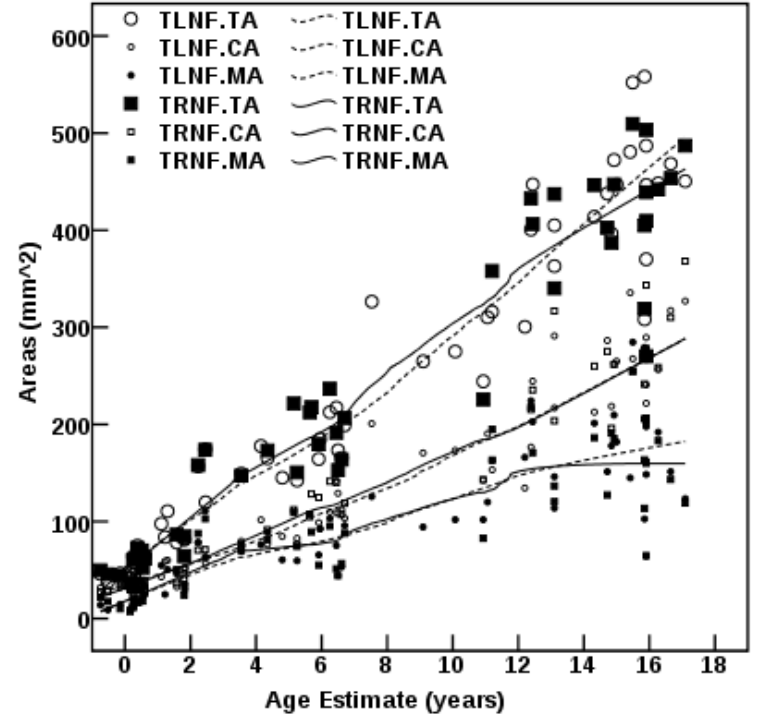

Figure 4.7. Areas (total, cortical, and medullary) of tibia nutrient foramen

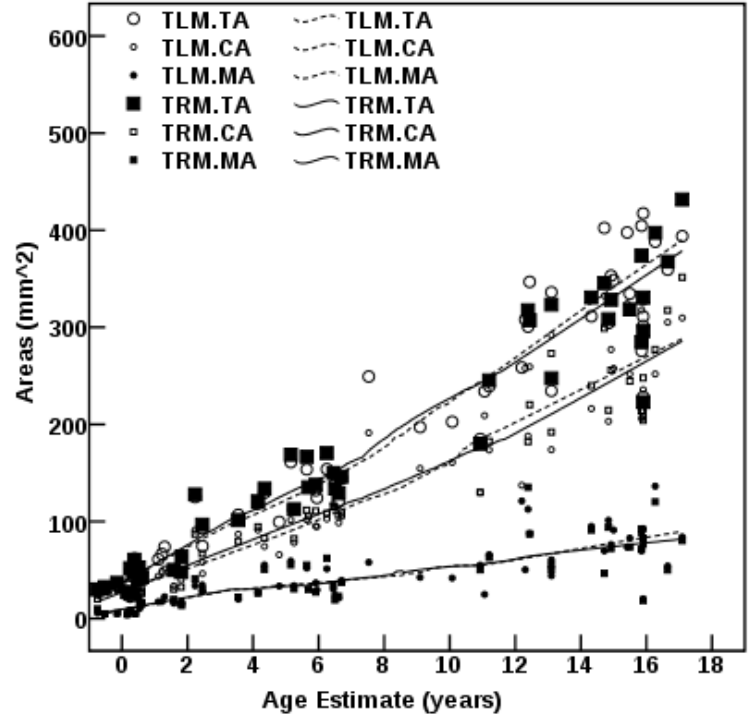

Figure 4.8. Areas (total, cortical, and medullary) of mid-shaft $(50 \%)$ tibia

LOESS curves for torsional rigidity at both the nutrient foramen (Figures 4.9) and mid-shaft (Figure 4.10) locations of the tibia follow a pattern similar to that observed in 
the femur. The rate of increase in strength has a higher slope after approximately seven years of age, suggesting a functional difference in torsional strength that emerges in late childhood. As in the femur, there is more variance in the later part of the developmental period in the proximal location than in the mid-shaft, and the nutrient foramen location has higher torsional rigidity values for the oldest individuals than the mid-shaft location. Absolute values for torsional rigidity in the tibia are similar to those the femur at the nutrient foramen location only. Mid-shaft torsional strength is appreciably lower than the proximal location in the tibia, in contrast to the femur where torsional rigidity values are equivalent in at the proximal and distal locations; this difference can be attributed to the proximal-distal tapering of the tibial circumference.
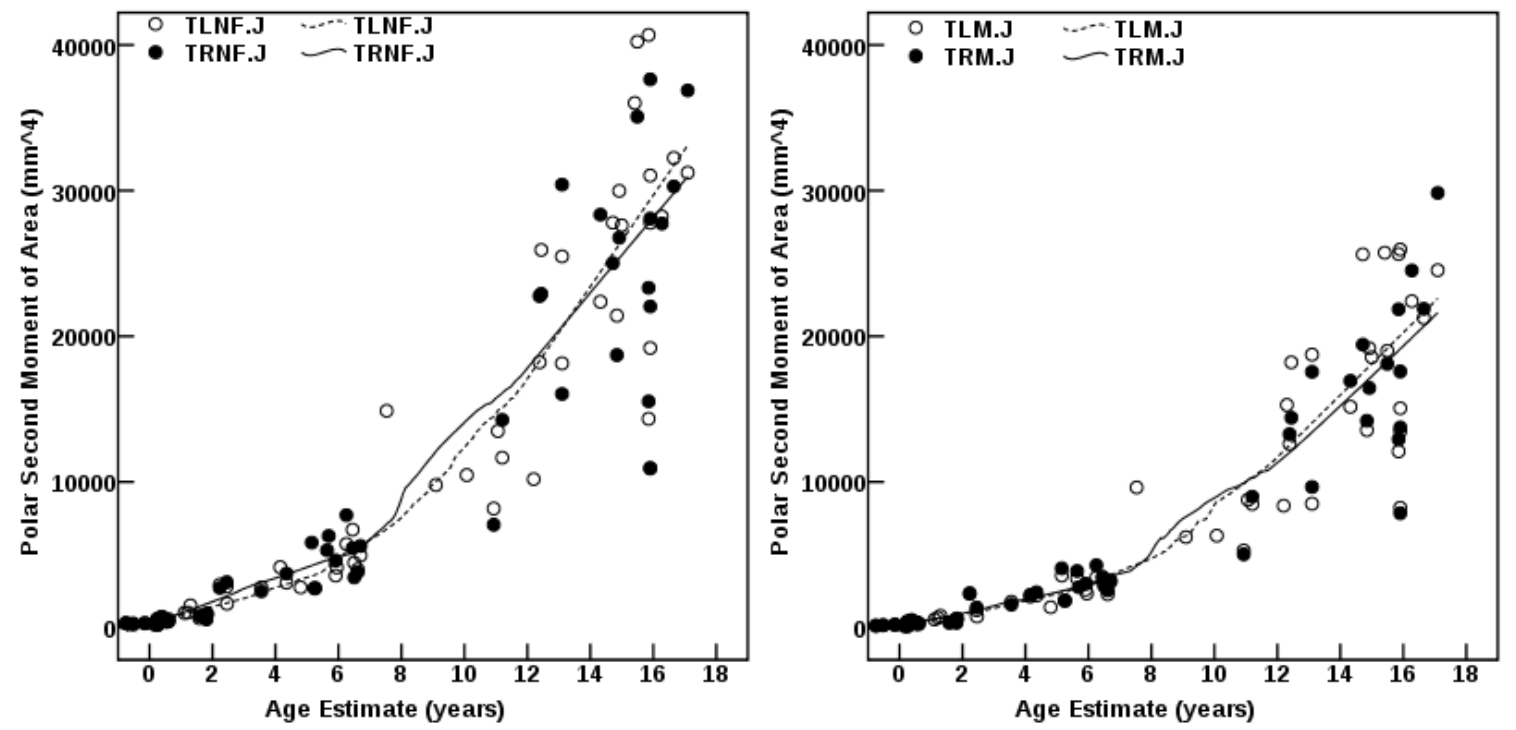

Figure 4.9. Torsional rigidity $(J)$ of the tibia nutrient foramen

Figure 4.10. Torsional rigidity (J) of the mid-shaft $(50 \%)$ tibia

With the mid-shaft torsional rigidity growth profile for the left tibia fit to a cubic model $\left(r^{2}=0.899\right)$, the pattern of unstandardized residuals illustrates an increasing amount 
of variability in strength levels from approximately age seven through to late adolescence (Figure 4.11). Among adolescents for whom sex could be estimated, females are distributed equally above and below the mean; and while five out six males are at or above the mean for torsional strength, there is no evidence to suggest a sex-based difference in tibial strength in individuals aged under 18 years. This pattern is the same observed for the femur: any sex-based differences in absolute leg strength in Later Stone Age adults is not yet present by late adolescence.

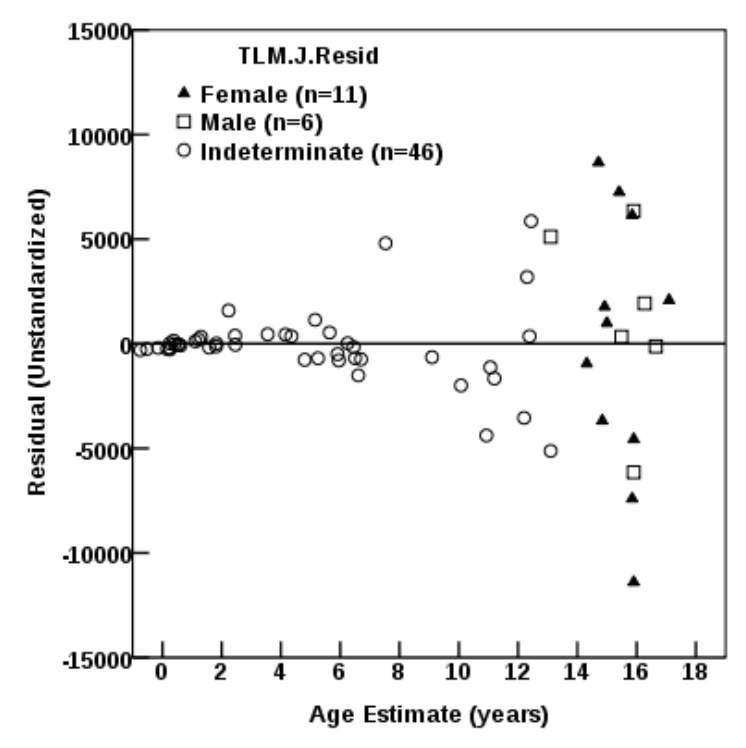

Figure 4.11. Unstandardized residuals for torsional rigidity $(\mathrm{J})$ of mid-shaft $(\mathbf{5 0} \%)$ left femur

\subsubsection{Cross-sectional properties of the clavicle}

Skeletal growth profiles for total, cortical, and medullary area of the mid-shaft clavicle (Figure 4.12) illustrate a pattern of increasing size, with no appreciable difference in values for left and right elements. Unlike the LOESS curves for areas of the 
femur and tibia, the trajectory for the clavicle does not appear to be strictly linear, but shows an increased slope after approximately age nine. The relative slopes for area values suggest that cortical area does not increase relative to total area with increasing age to the same degree as is observed for the lower limb.

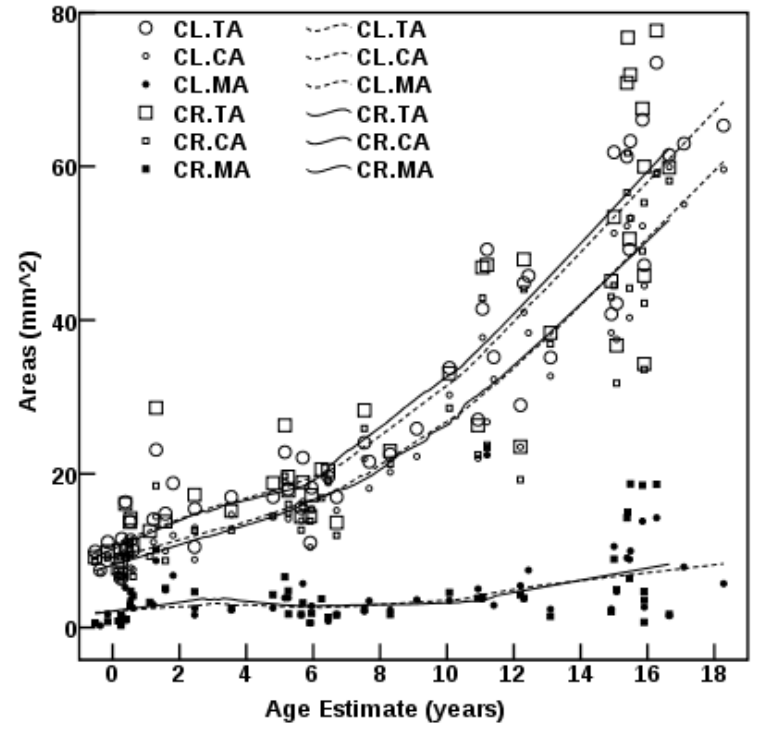

Figure 4.12. Areas (total, cortical, and medullary of the mid-shaft $(50 \%)$ clavicle

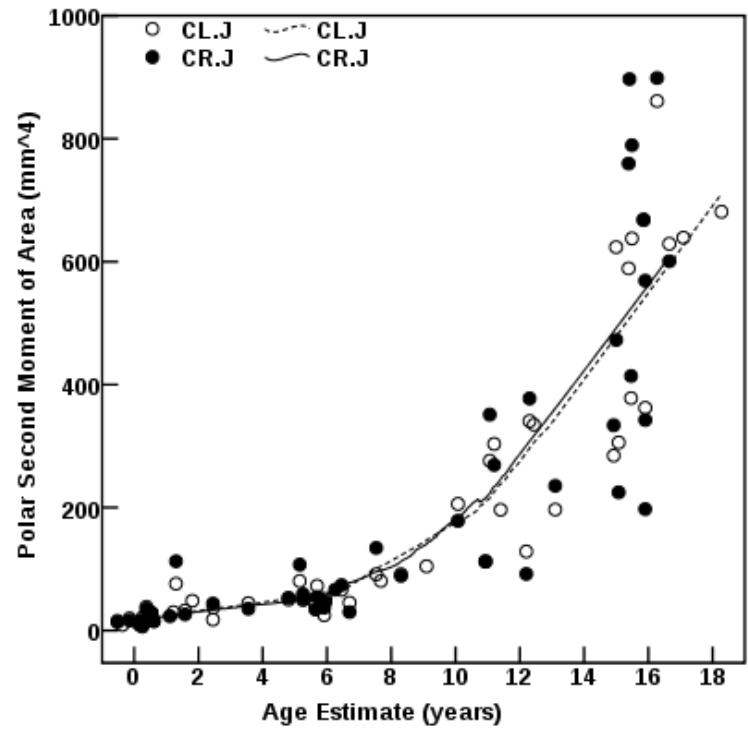

Figure 4.13. Torsional rigidity (J) of the mid-shaft $(50 \%)$ clavicle

Torsional strength in the clavicle increases significantly in individuals aged over nine years, from values under $200 \mathrm{~mm}^{4}$ to levels more than four times higher by late adolescence (Figure 4.13). Unstandardized residuals from cubic regression of the growth profile for the left clavicle (Figure 4.14), illustrate increasing levels of variability in torsional rigidity among juveniles aged approximately nine years and older. As is observed in the lower limb, torsional rigidity values are relatively stable among younger children and infants. The sex-based patterning of residuals for clavicular torsional strength is like that observed in the lower limb: females are equally distributed about the 
mean, and while males tend to have torsional strength values close to or above the mean, there is no clear indication of a sex-based difference in clavicular strength during adolescence.

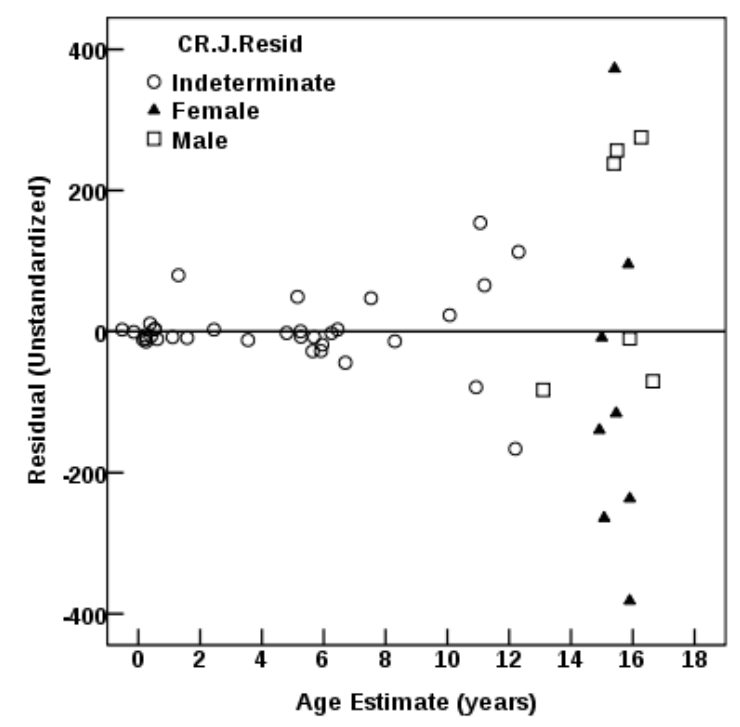

Figure 4.14. Unstandardized residuals for torsional rigidity $(J)$ of mid-shaft $(50 \%)$ right clavicle

\subsubsection{Cross-sectional properties of the humerus}

The skeletal growth profiles for areas of the mid-shaft (Figures 4.15) and distal (Figure 4.16) humerus increase with age in a pattern that, like the femur and tibia, appears linear from the LOESS curves. The relative slopes for total, cortical and medullary areas suggest a slight increase in cortical area as a proportion of total area over the course of development. Cortical area values for the mid-shaft humerus are more than three times those for the clavicle by late adolescence. Both the mid-shaft and distal humeral cross-section locations display a clear difference in left and right element values 
beginning in mid-childhood, with right element being larger on average than the left for the sample as a whole. The absolute magnitude of the difference between average left and right limb circumferences is, at most, approximately $15 \mathrm{~mm}^{2}$ among the late adolescents. Individual levels of asymmetry in left-right element pairs will be examined further in section 4.3. There is little difference in areas and therefore, in axial loading, between the mid-shaft and distal humeral cross-sections at any given age.
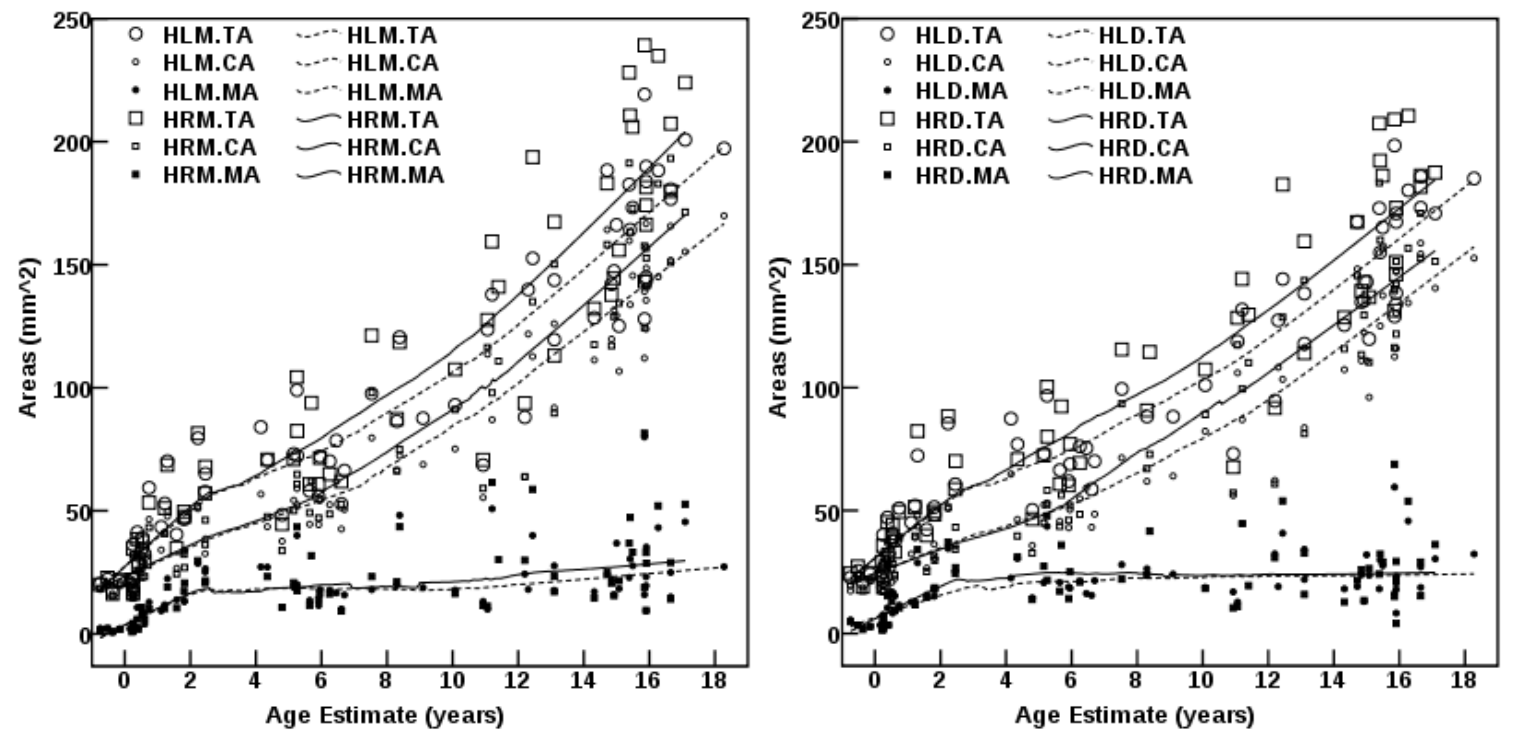

Figure 4.15. Areas (total, cortical, and medullary) Figure 4.16. Areas (total, cortical, and medullary) of the mid-shaft $(\mathbf{5 0} \%)$ humerus of the distal (35\%) humerus

The LOESS curves for torsional strength in the mid-shaft (Figure 4.17) and distal (Figure 4.18) humerus indicate a pattern for increasing strength with age that is not strictly linear; beginning in mid-childhood there is a pattern of increasing levels of gain in strength with each subsequent age group. In contrast to the axial strength measures which are approximately equivalent at the mid-shaft and distal locations, torsional strength is appreciably higher in the mid-shaft location among older adolescents, which is 
due almost entirely to right element values. As with area measurements, there is a pattern of increased torsional strength in right elements relative to left humeri across the entire sample in individuals aged approximately six years and older.
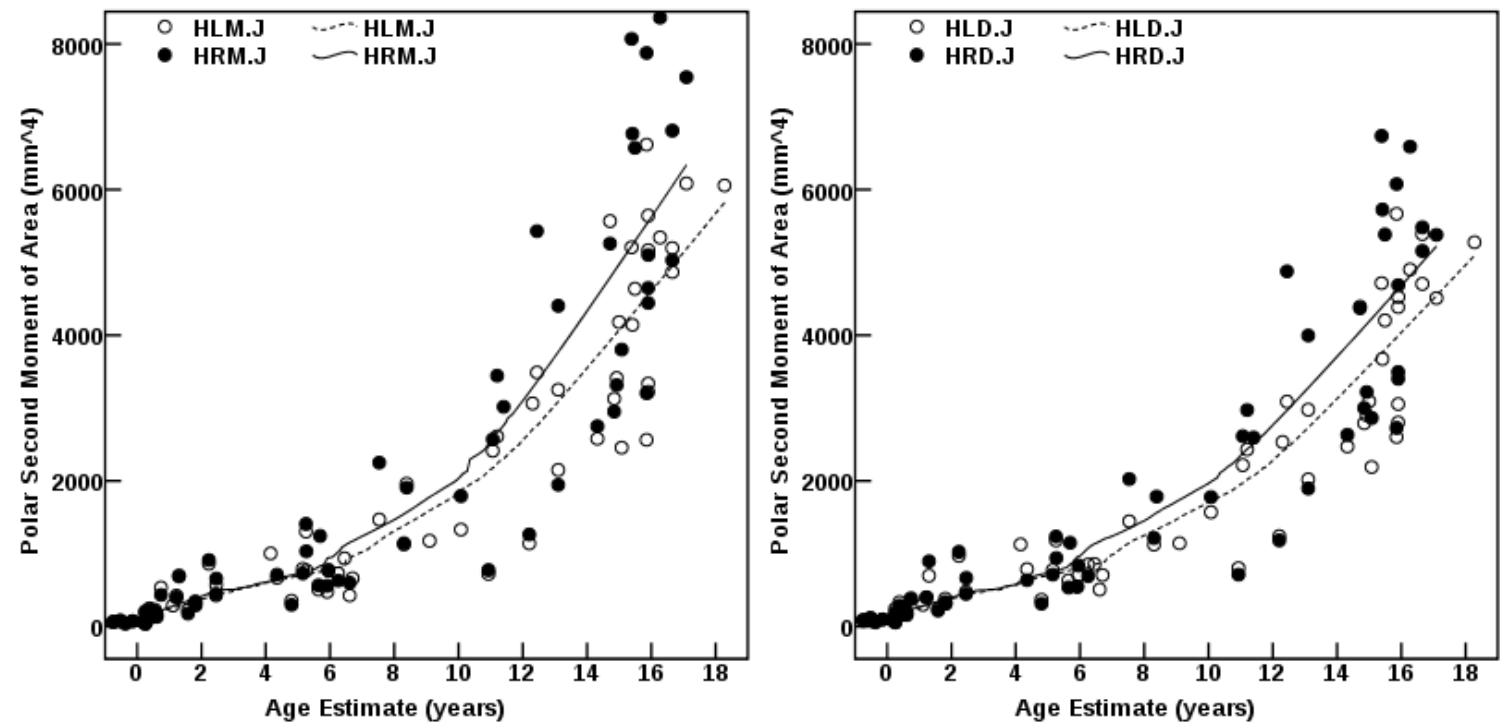

Figure 4.17. Torsional rigidity $(\mathrm{J})$ of the mid-shaft Figure 4.18. Torsional rigidity $(\mathrm{J})$ of the distal $(50 \%)$ humerus $(35 \%)$ humerus

Approximately 20 percent of adult torsional strength for the right humerus is achieved by six years of age among Later Stone Age juveniles (Figure 4.19). The majority of torsional strength is accrued during adolescence, with the adult mean achieved among some of the oldest adolescents in the sample. Unstandardized residuals from regression of the skeletal growth profile for torsional strength of the right humerus using a cubic model (Figure 4.20) illustrates an increase in variability beginning in late childhood. Sex estimates for the adolescents illustrate a tendency for males to have stronger right humeri than average, though many females have torsional strength values in the range of males. As is observed for the lower limb, any clear sex-based difference 
in the magnitude of torsional strength in the humerus is not present in late adolescence among Later Stone Age hunter-gatherers.
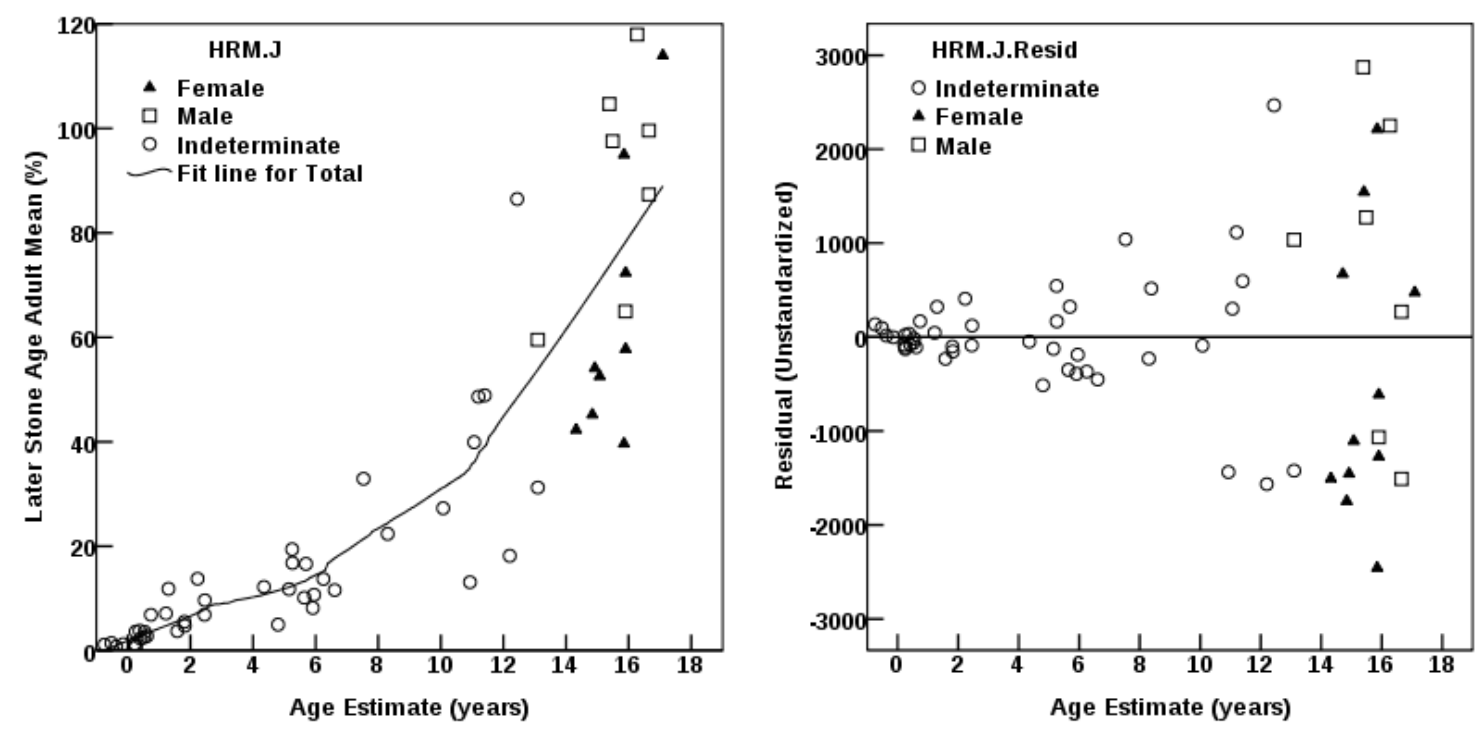

Figure 4.19. Torsional rigidity $(\mathrm{J})$ of the mid-shaft Figure 4.20. Unstandardized residuals for $(50 \%)$ right humerus standardized to the adult Later Stone Age population mean torsional rigidity $(\mathrm{J})$ of mid-shaft $(\mathbf{5 0} \%)$ right humerus

\subsubsection{Cross-sectional properties of the ulna}

The LOESS curves for the skeletal growth profiles of area measurements of the ulna (Figure 4.21) illustrate that the element increases in size in a linear fashion throughout development. The relative slopes of the area trajectories suggests that cortical area increases relative to total area with increasing age. Areas of the ulna are about half the size of those for the humerus at any given age, suggesting that the distal segment of the arm is subject to axial loads half as great as those borne by the humerus. Area values for the left and right ulnae diverge somewhat later than in the humerus, in individuals aged approximately eight years and older, with the right ulna being larger in 
circumference, on average, than the left across the sample.

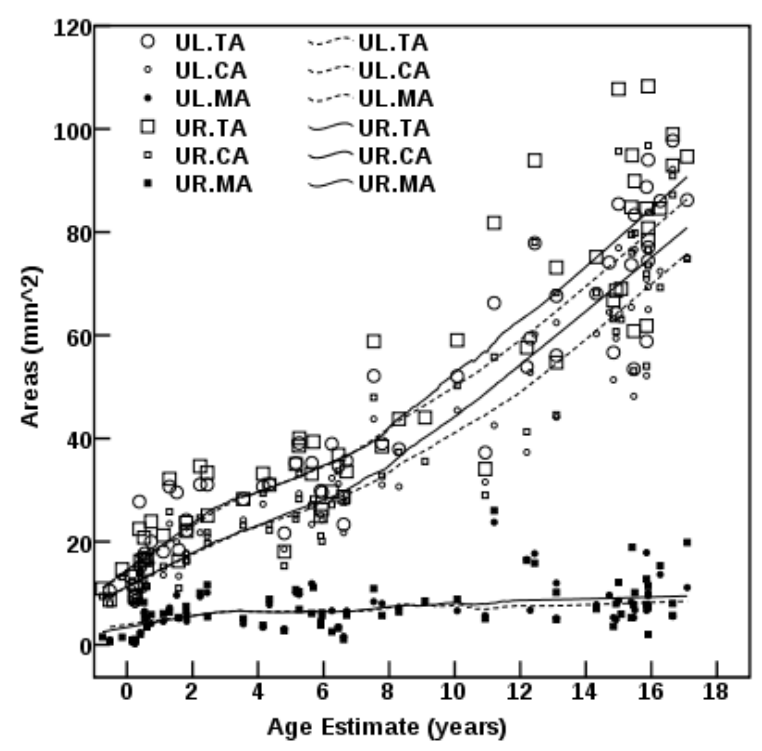

Figure 4.21. Areas (total, cortical, medullary) of the mid-shaft $(50 \%)$ ulna

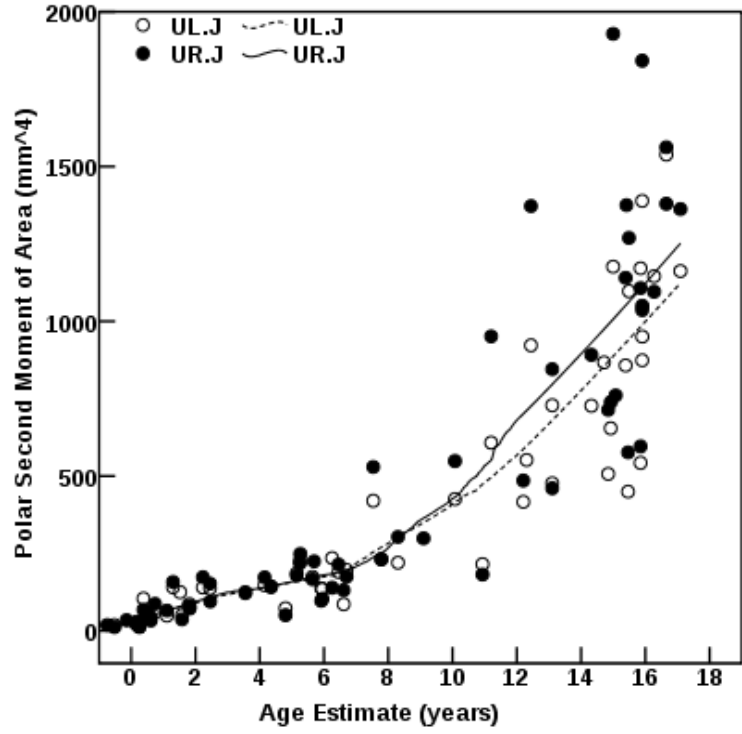

Figure 4.22. Torsional rigidity (J) of the mid-shaft $(50 \%)$ ulna

Torsional strength increases are small among individuals aged six years and younger, with a much greater slope to the trajectory for the remainder of the growth profile. As with the area measurements, the right ulna is consistently stronger than the left, on average, among individuals aged eight years and older. The onset of strength asymmetry coincides with an increase in variability in torsional strength, as illustrated by the residuals from a regression of the skeletal growth profile for the right mid-shaft ulna using a cubic model (Figure 4.23). Sex estimates among the adolescents suggest a tendency for male ulnar strength to be greater than that for females, on average, though some females are within the range of males in late adolescence. 


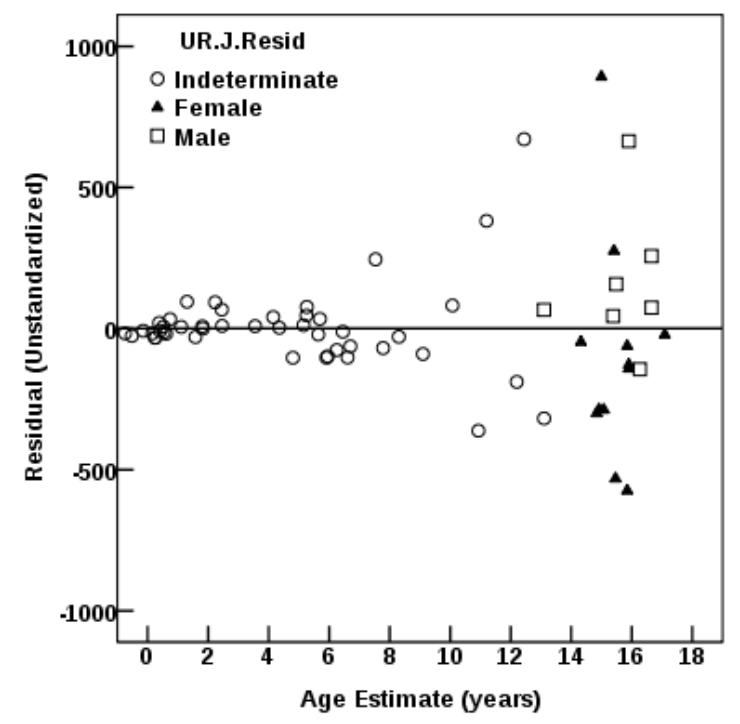

Figure 4.23. Unstandardized residuals for torsional rigidity $(J)$ of mid-shaft $(50 \%)$ right ulna

\subsubsection{Summary of skeletal growth profiles for postcranial robusticity}

Visual inspection of LOESS curves from skeletal growth profiles suggest that areas increase in a linear fashion while polar second moments of area increase in an exponential pattern; the difference between trajectories is expected given the units of measurement for areas $\left(\mathrm{mm}^{2}\right)$ and polar second moments of area $\left(\mathrm{mm}^{4}\right)$. There is a general increase in cortical area relative to total area over the course of development in all elements. For all properties and in all elements studied, there is no sign of a plateau in values by late adolescence in any element, indicating that appositional growth continues into early adulthood among Later Stone Age hunter-gatherers. Axial and torsional strength are closely related to age, increasing modestly, prior to mid-childhood. Leg strength measures increase in variability after approximately six years of age, while 
variability in arm strength measures seems to increase somewhat later in development, after approximately eight years of age.

The absolute magnitude of all cross-sectional properties tend to be slightly greater in the femur than in the tibia, suggesting that the proximal segment of the lower limb bears more axial and torsional loads than does the distal segment. Within the femur and the tibia, the proximal cross-section locations are greater in circumference than the midshaft, but bear less axial force, as indicated by the proportion of cortical tissue. Torsional strength, however, is borne equally in the proximal and mid-shaft regions of both the femur and the tibia. In the humerus, axial strength is equal at mid-shaft and at the distal cross-section location, while torsional strength is greater at mid-shaft. Individual relative limb strength will be explored further in section 4.2 .

Growth profiles illustrate a trend for greater strength in the right humerus and ulna over left elements across the whole sample. No appreciable side differences are observed in the clavicle or lower limb. Individual side asymmetry will be evaluated in section 4.3 . Comparisons of mid-shaft torsional strength of the femur and humerus to mean values for Later Stone Age adults illustrate that the majority of torsional strength is accrued during adolescence. There is no clear pattern for sex-based differences in limb strength by late adolescence, although males tend to have absolute strength values at or above the mean, particularly in the humerus and ulna. 


\subsection{Strength proportions in the upper and lower limb}

Torsional rigidity was analysed from the perspective of the relative development of robusticity between the upper and lower limb, and between proximal and distal segments within limbs. Ratios of polar second moments of area were plotted against estimated age at death for the following element pairs: femur/humerus, tibia/humerus, femur/tibia, humerus/ulna, and humerus/clavicle. The data represent relative limb strength within individuals, requiring preservation of both elements; left and right element pairs were analysed. LOESS curves were fit to aid in identifying developmental patterns in the skeletal growth profiles. Additional curve fitting was performed to determine the strength and nature of the relationship between estimated age at death and the dependent variables. In all cases, a cubic polynomial was the best fit for regression of proportional strength measures on estimated age at death.

\subsubsection{Lower to upper limb strength proportions}

The relationship between lower and upper limb strength was evaluated in terms of both the tibia and femur relative to the humerus. During infancy femoral strength is about 2.5 times that of the femur, on average (Figure 4.25). Between two and six years of age, femoral strength increases rapidly relative to the humerus, and by around ten years of age ceases to increase further. There is some evidence for a decrease in relative femoral to humeral strength in late adolescence, due to the larger gains in humeral over femoral strength during late adolescence. The skeletal growth profiles for mid-shaft torsional strength above illustrated more symmetry in femoral than in humeral strength 
across the sample as a whole with right humeri observed to be stronger, in general, than left. This trend plays out within individual femur-humerus pairs as differences in proportional strength, with appreciable divergence in proportions from age six onward. During adolescence, the right femur is approximately four times stronger than the right humerus, while the weaker left humerus is evident from left femoral strength that is approximately five times greater.

Unstandardized residuals from a cubic regression of right femur-humerus proportional torsional strength on estimated age (Figure 4.24) illustrate lower variability among individuals aged two years and younger, and higher variability in proportional lower to upper limb strength throughout the rest of development. Sex estimates among the adolescents suggest a tendency for males to have stronger right humeri relative to the femur, but approximately half of the female adolescents in the sample are also within this range for proportional strength.
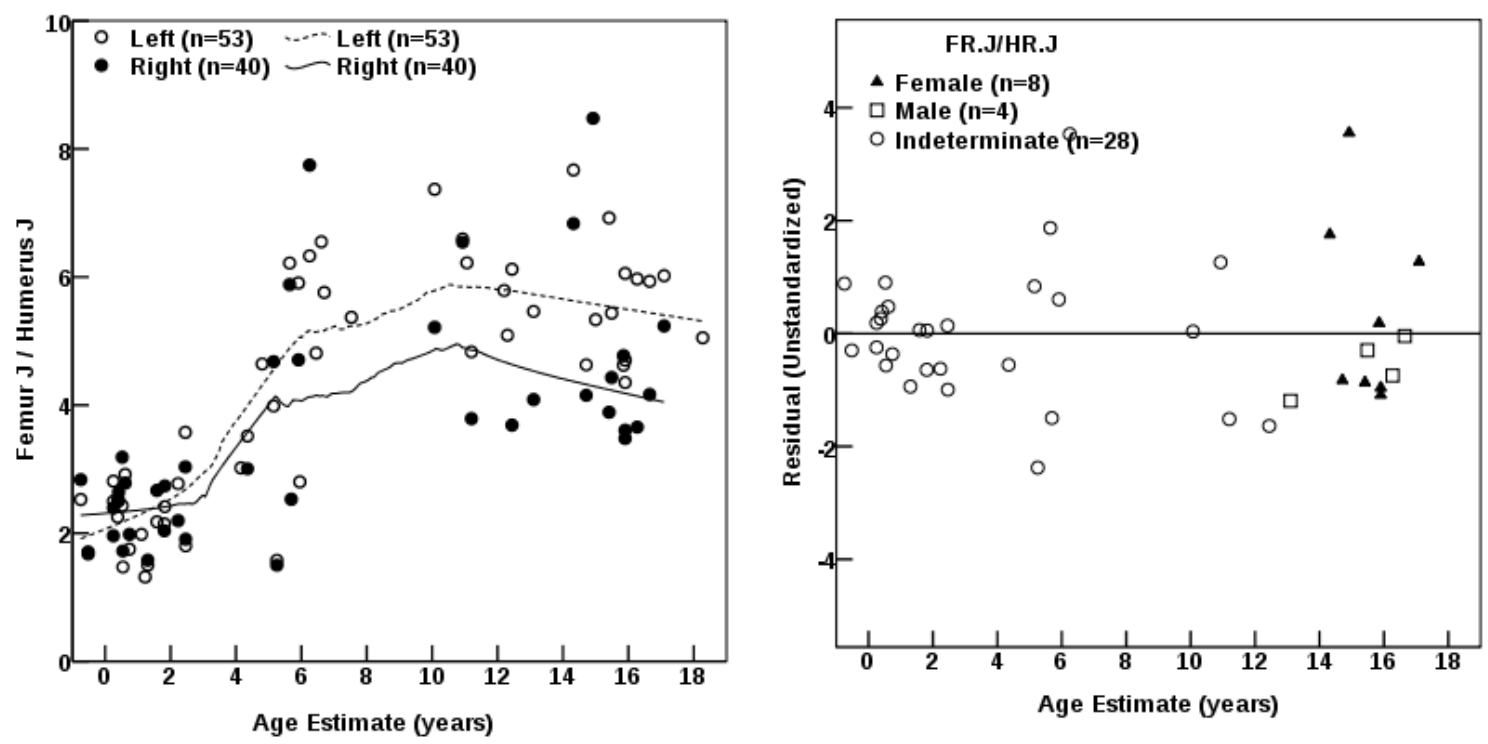

Figure 4.25. Individual proportional torsional Figure 4.24. Unstandardized residuals for cubic rigidity $(J)$ of mid-shaft femur relative to humerus regression of right femoral to humeral proportional torsional rigidity $(J)$ on estimated age 
The tibia follows a pattern similar to the femur in torsional rigidity relative to the humerus, with a strong increase in strength relative to the humerus after two years of age, that reaches a plateau by approximately age six (Figure 4.26). The skeletal growth profiles for the tibia above, illustrated that torsional strength is slightly weaker in the tibia than in the femur, and this difference is reflected in proportional strength. Not only is the magnitude of tibial to humeral proportional strength slightly lower than that of the femur, but the smaller divergence between left and right pairs also reflects the weaker tibia. The relative strengthening of the humerus over the tibia in late adolescence is marked by the greater decrease from the plateau in the tibial proportional growth profile than observed for the femur. Unstandardized residuals from a cubic regression of right tibia to humeral relative torsional rigidity on age (Figure 4.27) illustrate a slight increase in variability in this measurement after approximately age two that continues throughout the remainder of the developmental course. There is no evidence for a sex-based difference in proportional lower to upper limb strength among adolescents as evidenced by an equal distribution of females and males around the residual mean. 


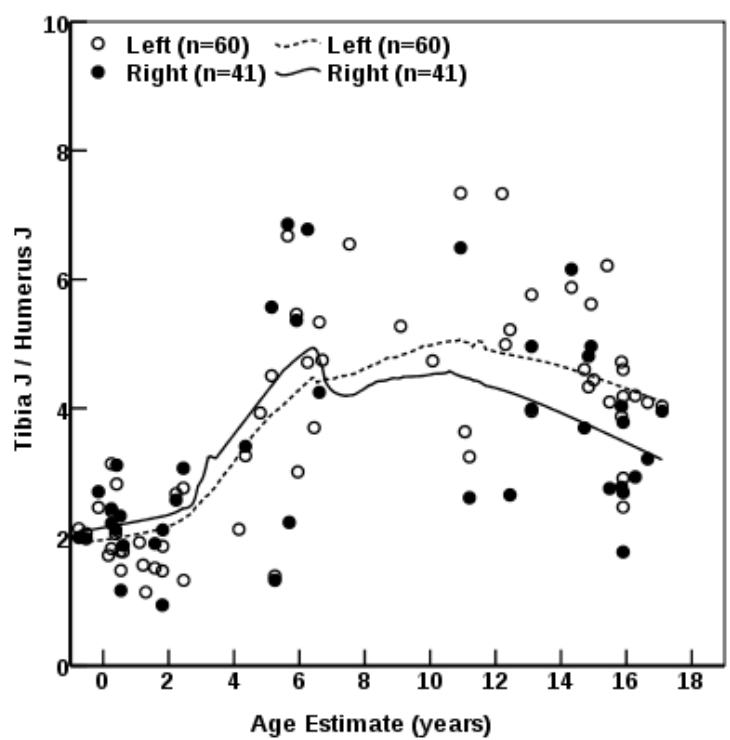

Figure 4.26. Individual proportional torsional rigidity $(J)$ of mid-shaft tibia relative to humerus

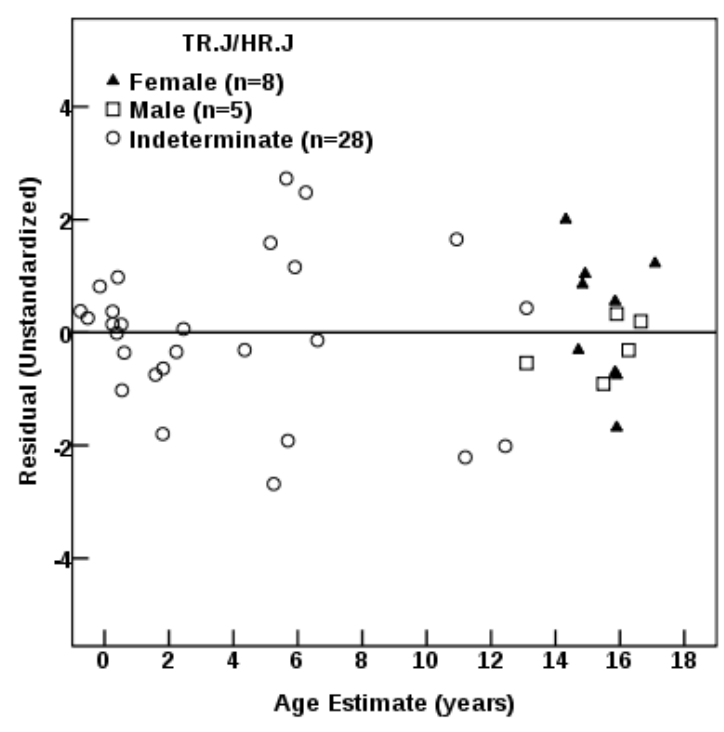

Figure 4.27. Unstandardized residuals for cubic regression of right tibial to humeral proportional torsional rigidity $(J)$ on estimated age

\subsubsection{Within-limb strength proportions}

Torsional strength proportions within the lower and upper limb are evaluated through an analysis of skeletal growth profiles for femur-tibia, humerus-ulna, and humerus-clavicle pairs within individuals. Strength proportions in the lower limb (Figure 4.28) are relatively constant throughout development, with the femur being slightly stronger than the tibia, on average. The profiles for left- and right-side pairs are similar, though with more variation among neonates. Prior to age six, many individuals have stronger torsional strength in the tibia than in the femur, with femur-tibia ratios of approximately 0.8 , but with increasing age most individuals have stronger proximal segments of the lower limb (femora) than distal segments (tibiae). There is evidence for continued strengthening of the femur relative to the tibia in late adolescence, with no 
indication that appositional growth ceases with early adulthood. A plot of unstandardized residuals from a cubic regression of relative femoral-tibial torsional rigidity on estimated age (Figure 4.29) illustrates that there is no appreciable age-related variation in lower limb strength proportions. Sex estimates among the adolescents show a tendency for males to have stronger tibiae relative to femora than females, although a number of females also display this characteristic; there is no clear sex distinction in lower limb proportional strength.

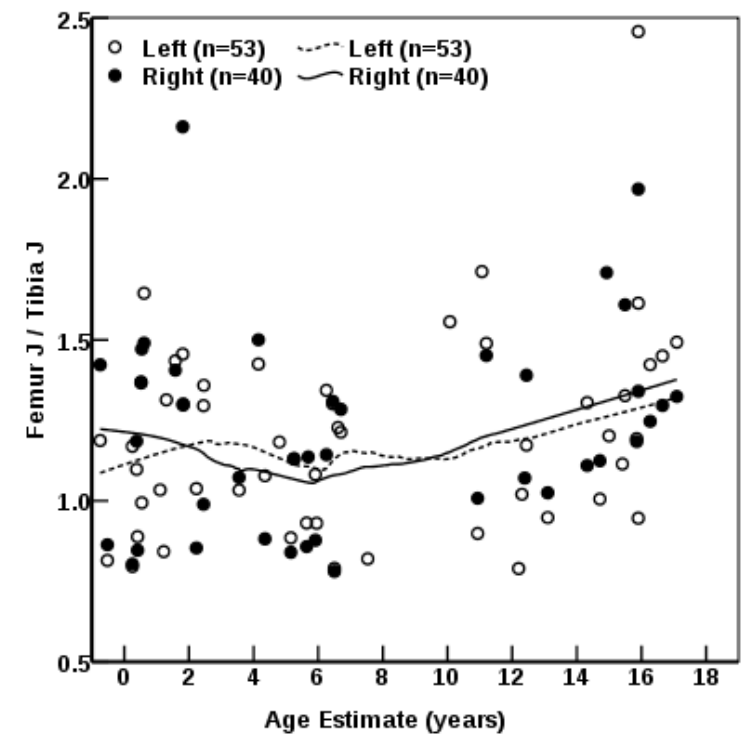

Figure 4.28. Individual proportional torsional rigidity $(J)$ of mid-shaft femur relative to tibia

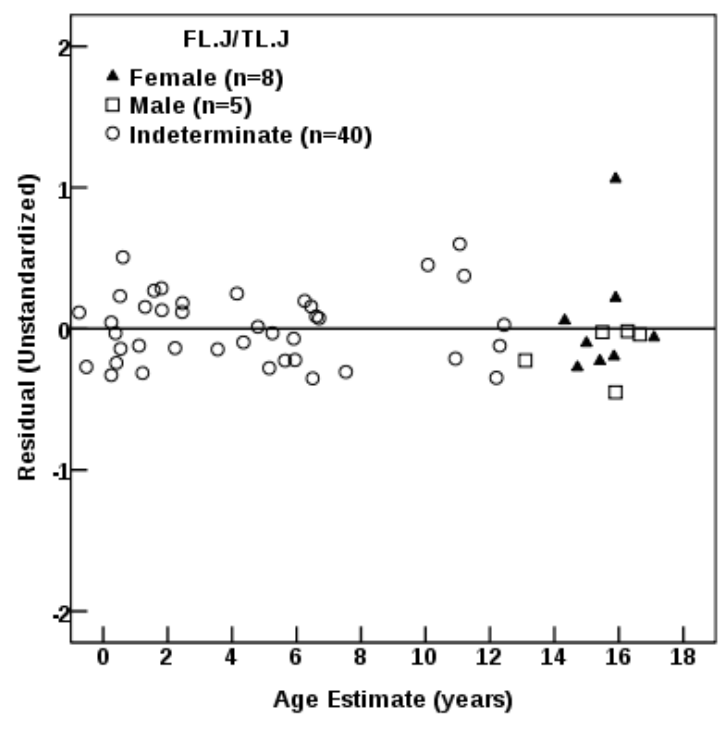

Figure 4.29. Unstandardized residuals for cubic regression of left femoral to tibial proportional torsional rigidity $(J)$ on estimated age

The skeletal growth profile for proportional strength in individual humerus-ulna pairs (Figure 4.30) illustrates that the humerus is, on average, approximately four times as strong as the ulna. Humeral strength increases markedly relative to the ulna between birth and approximately four years of age, to a peak ratio of about 5:1. During mid- to 
late-childhood, the ulna gains strength relative to the humerus, and during adolescence humeral strength appears to go through another phase of marked increase in proportional strength. Profiles for the left- and right-limb pairs are equivalent in the pattern and magnitude of the trajectory with the exception of the mid- to late-childhood phase of decline in proportional strength. The divergence observed during this phase is attributed to the fact that asymmetry in the ulna develops later than that of the humerus, as observed in the skeletal growth profiles for torsional strength in these elements above. The right humerus is, on average, stronger than the left humerus beginning at age six, while the right ulna remains equivalent in strength to the left until approximately eight to ten years of age. There is no age-related pattern to the variability observed in humerus-ulna proportional strength (Figure 4.31). There is some evidence for a tendency for the ulna to be stronger relative to the humerus in males, as indicated by the distribution of residuals for males and females among the adolescents in the sample. 


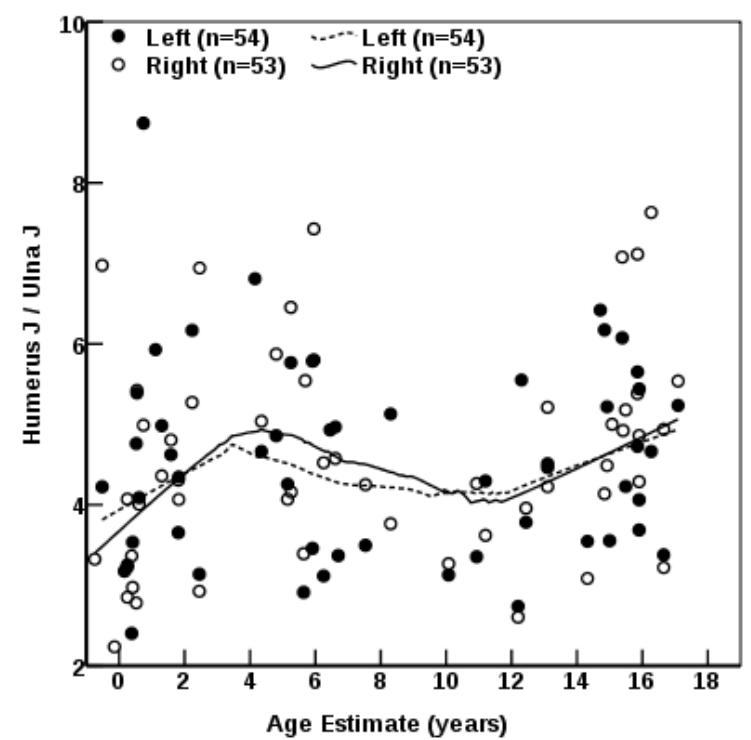

Figure 4.30. Individual proportional torsional rigidity $(J)$ of mid-shaft humerus relative to ulna

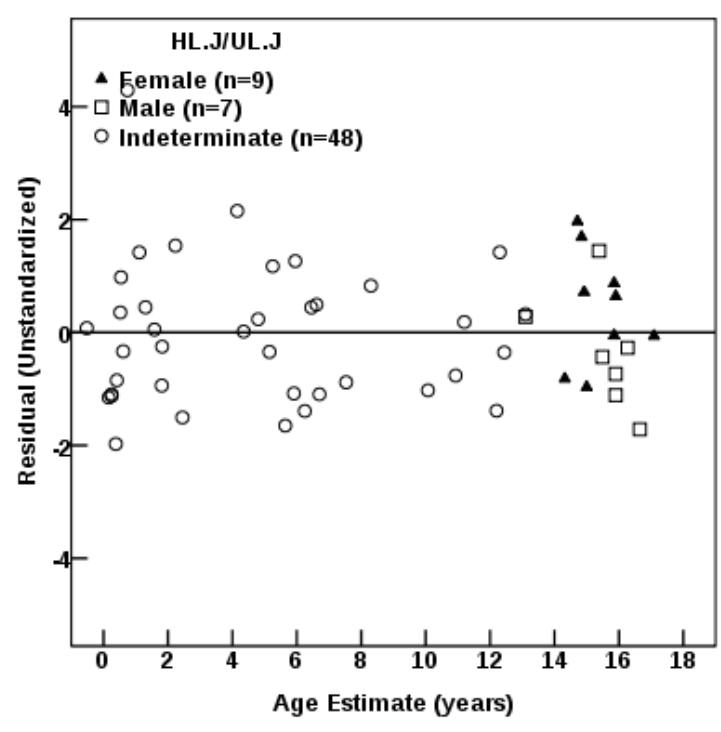

Figure 4.31. Unstandardized residuals for cubic regression of left humeral proportional torsional rigidity $(J)$ on estimated age

Torsional strength in the humerus increases markedly relative to the clavicle during the initial phase of the growth profile as was observed for proportional strength in the humerus relative to the ulna. The clavicle is, however, one tenth as strong as the humerus on average, with a peak ratio of 15:1 at approximately among individuals aged six years. The clavicle gains strength relative to the humerus during late childhood, and by adolescence has plateaued at about one tenth of humeral torsional rigidity. Unlike the other proportional strength relationships observed, there is no change in relative strength between the humerus and clavicle after age twelve. Because the skeletal growth profiles for torsional strength in the humerus and ulna illustrate continued appositional growth into young adulthood, the plateau in relative humerus-clavicle strength indicates that these limb segments increase in strength proportionally during adolescence and beyond. 
Proportional strength in right humerus-clavicle pairs is greater than in the left side in individuals aged six years and older because there is asymmetry in humeral torsional strength but none in the clavicle, as indicated by the skeletal growth profiles above for these elements. Unstandardized residuals for a cubic regression of left humerus-clavicle proportional strength on estimated age (Figure 4.33) illustrate a much greater magnitude of variability in this element pair relative to the others studied. There is no evidence for a sex-based difference in proportional humeral strength relative to the clavicle.

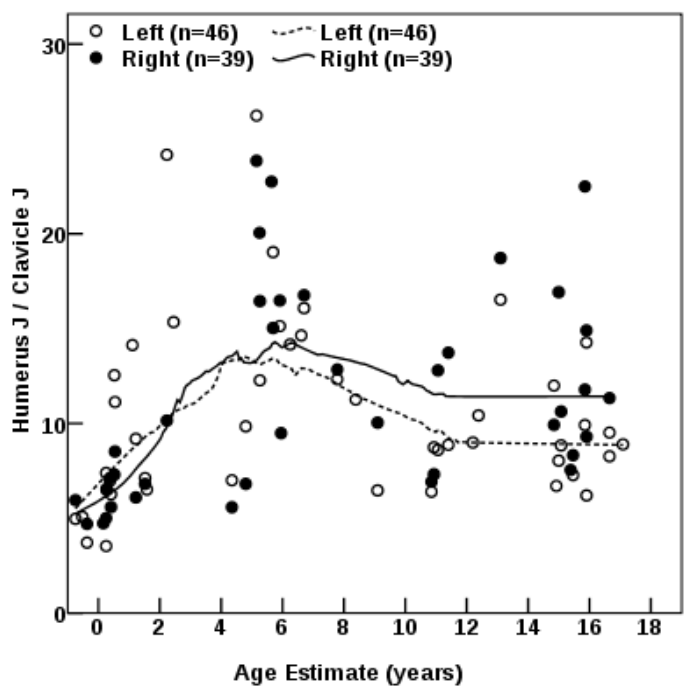

Figure 4.32. Individual proportional torsional rigidity $(J)$ of the mid-shaft humerus relative to clavicle

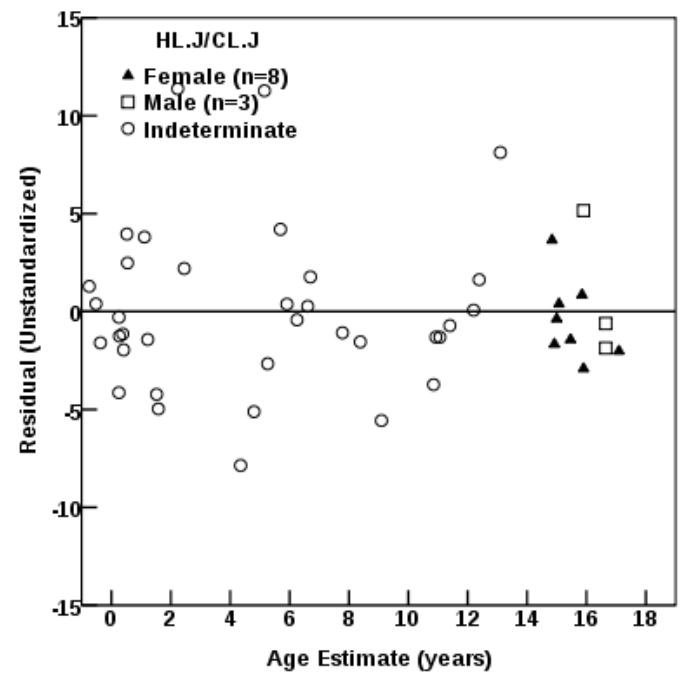

Figure 4.33. Unstandardized residuals for cubic regression of left humeral to clavicular proportional torsional rigidity $(J)$ on estimated age

\subsubsection{Summary of proportional strength measures}

Analysis of proportional strength measures for both the femur-humerus and tibiahumerus pairs illustrates that lower limb strength increases markedly relative to the upper limb between two and six years of age. Humerus strength appears to "catch up" relative 
to the femur during mid- to late-childhood and exceeds gains in femoral strength during adolescence. While both the upper and lower limb are continually strengthened into late adolescence among Later Stone Age hunter-gatherers, gains in humeral strength tend to occur later (mid-childhood and onward). Put another way, proportional strength measures indicate that the significant change in femur strength occurs between two and six years of age.

Analysis of strength proportions between limb segments illustrates that segments within the upper limb change in relative strength over the course of development, while in the lower limb, femoral to tibial strength is fairly stable throughout ontogeny. Both the clavicle and the ulna lag behind the humerus in torsional strength accrual in the initial period of the growth trajectory, but gain strength relative to the humerus during mid- and late-childhood. During adolescence strength gains in the ulna slow while the clavicle keeps pace with the humerus. Both proximal limb segments (femur and humerus) gain strength relative to their distal counterparts (tibia and ulna) in adolescence, and presumably into early adulthood. There may be a sex-based distinction in this trend, as there is some evidence for relatively weaker distal limb segments among females.

\subsection{Biomechanical shape and asymmetry}

Biomechanical shape, a measure of proportional bending strength, is evaluated through two ratios of perpendicular second moments of area: the medio-lateral relative to the antero-posterior axis $\left(\mathrm{I}_{\mathrm{x}} / \mathrm{I}_{\mathrm{y}}\right)$, and the major relative to the minor bending axis 
(Imax/Imin). These ratios evaluate shape in the sense that the distribution of cortical tissue about the centroid determines bending strength; for a circular hollow beam, all shape ratios of perpendicular axes will be equal to one. The ratio of medio-lateral to anteroposterior bending strength $\left(\mathrm{I}_{\mathrm{x}} / \mathrm{I}_{\mathrm{y}}\right)$ is plotted against estimated age for all cross-section locations. Additional shape analyses are performed for the mid-shaft femur and humerus through the ratio of the maximal and minimal second moments of area (Imax/Imin) derived from the latex cast method (LCM). Because the LCM assess the true subperiosteal contour, the magnitude of $I_{\max }$ and its orientation relative to the medio-lateral axis $\left(\mathrm{I}_{\mathrm{x}}\right.$, taken counter-clockwise by convention) can be measured. Individual asymmetry in torsional strength between pairs of elements is also evaluated using percentage, calculated as [(stronger element - weaker element) / weaker element] x 100. Results for biomechanical shape and asymmetry are presented together for the lower limb, followed by the upper limb.

\subsubsection{Shape and asymmetry of the lower limb}

Ratios of $\mathrm{I}_{\mathrm{x}} / \mathrm{I}_{\mathrm{y}}$ bending strength for the femur reflect shape differences in the proximal and mid-shaft areas of the femoral diaphysis over the course of growth. The subtrochanteric cross-section is highly variable throughout the entire developmental trajectory (Figure 4.34), relative to the mid-shaft (Figure 4.35) due to the irregularity in this cross-sectional location described above. The proximal region of the femur is, however, proportional with respect to the $\mathrm{x}$ - and $\mathrm{y}$-axes, on average, among infants and then becomes medio-laterally ovoid through adolescence. In individuals aged twelve 
years and older, the subtrochanteric cross-section returns to a more circular shape, on average. These shape changes reflect an adaptation to greater bending forces in the antero-posterior plane, followed by an increase in loading in the medio-lateral plane. Similar growth profiles are observed for the left and right femora.

The mid-shaft cross-section, in contrast, is medio-laterally ovoid and highly variable in shape among individuals aged approximately two years and younger. The shape of the mid-shaft femur becomes increasingly larger along the antero-posterior axis, throughout the remainder of the growth trajectory, reflecting increased medio-lateral bending strength. Variability in mid-shaft biomechanical shape decreases noticeably after two years of age and remains stable throughout childhood; with the onset of adolescence, however, there is increased variability in biomechanical shape measures. As with the subtrochanteric cross-section, the left and right mid-shaft growth profiles for the femur are nearly identical. 


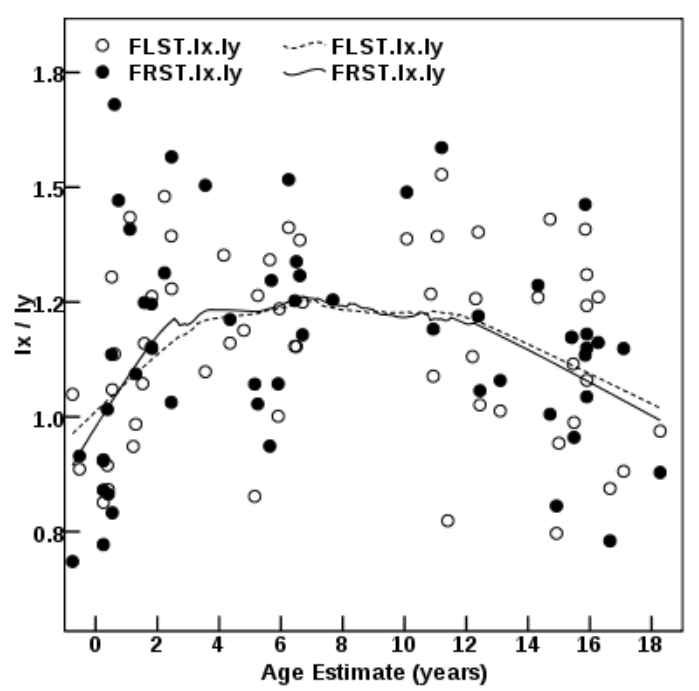

Figure 4.34. Biomechanical shape $\left(I_{x} / I_{y}\right)$ of the subtrochanteric $(80 \%)$ femur

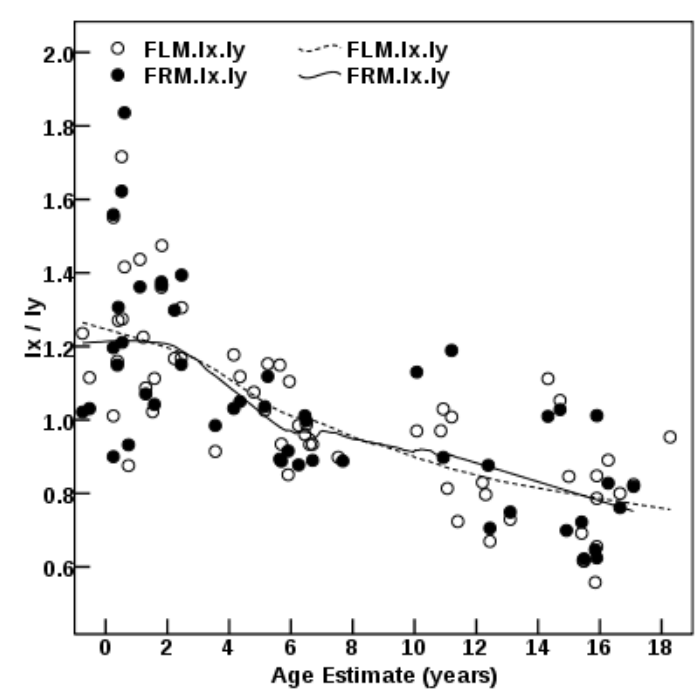

Figure 4.35. Biomechanical shape $\left(I_{x} / I_{y}\right)$ of the mid-shaft $(50 \%)$ femur

The ratio of maximal to minimal bending strength in the mid-shaft femur is highly variable among individuals aged two years and younger, but stabilizes throughout childhood (Figure 4.36). The resulting LOESS curve for the growth profile suggests a decline in magnitude of maximal bending strength relative to the minimum, between birth and age six; this is in keeping with the profile for $\mathrm{I}_{\mathrm{x}} / \mathrm{I}_{\mathrm{y}}$. Throughout the remainder of the developmental trajectory, the mid-shaft femur becomes increasingly less circular with bending strength levels 1.5 times greater than the minimum on average, among the late adolescents, though with more variability observed in this age group. The scatterplot for the orientation of the axis of maximal bending strength relative to the mediolateral axis confirms the observations of $\mathrm{I}_{\mathrm{x}} / \mathrm{I}_{\mathrm{y}}$ and $\mathrm{I}_{\max } / \mathrm{I}_{\min }$ : with development there is a trend toward expansion of the mid-shaft femur along the antero-posterior axis. Figure 4.37 illustrates a convergence of the orientation of $\operatorname{Imax}$ on $90^{\circ}$ as appositional growth 
proceeds.
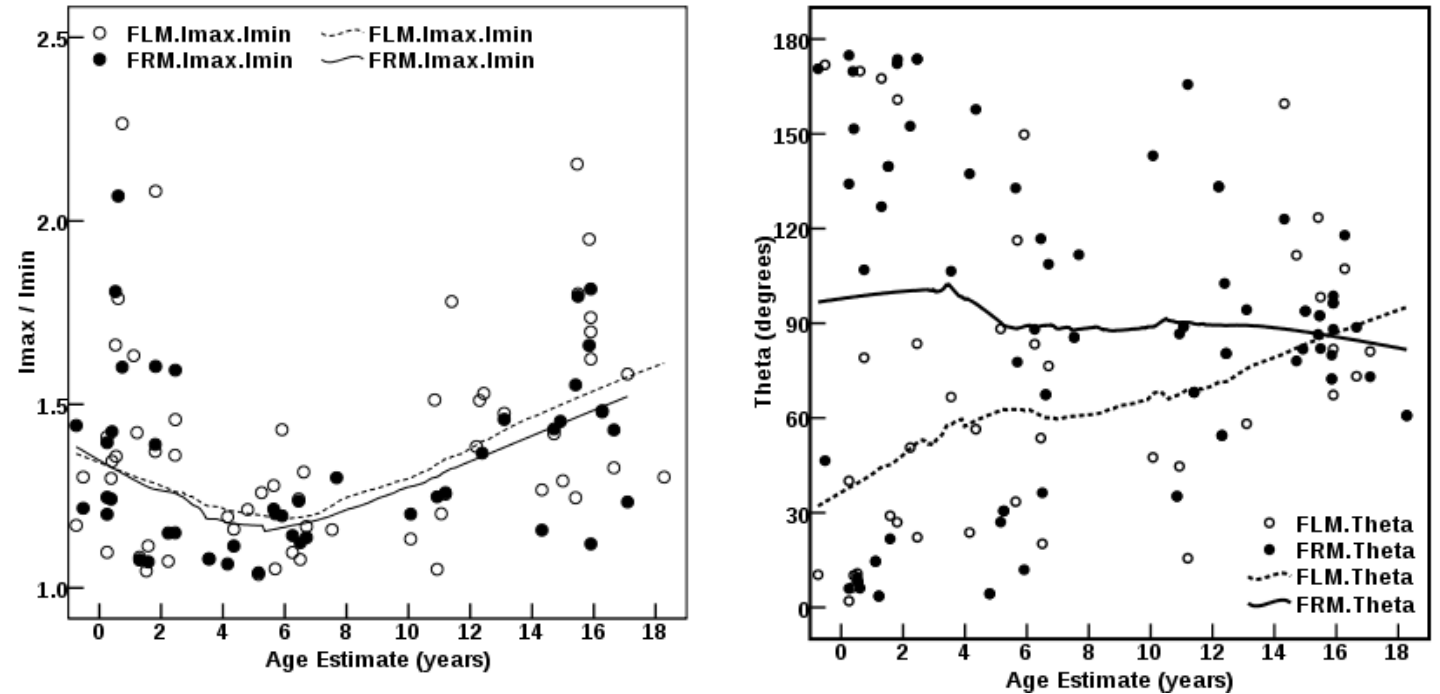

Figure 4.36. Biomechanical shape $\left(I_{\max } / I_{\min }\right)$ of the Figure 4.37. Orientation of the axis of maximal mid-shaft femur bending strength (theta) of the mid-shaft $(50 \%)$ femur

There is no evidence for sex-based shape differences in the mid-shaft femur, with an even distribution of female and male adolescents about the LOESS curve for maximum bending rigidity in the left mid-shaft femur (Figure 4.38). 


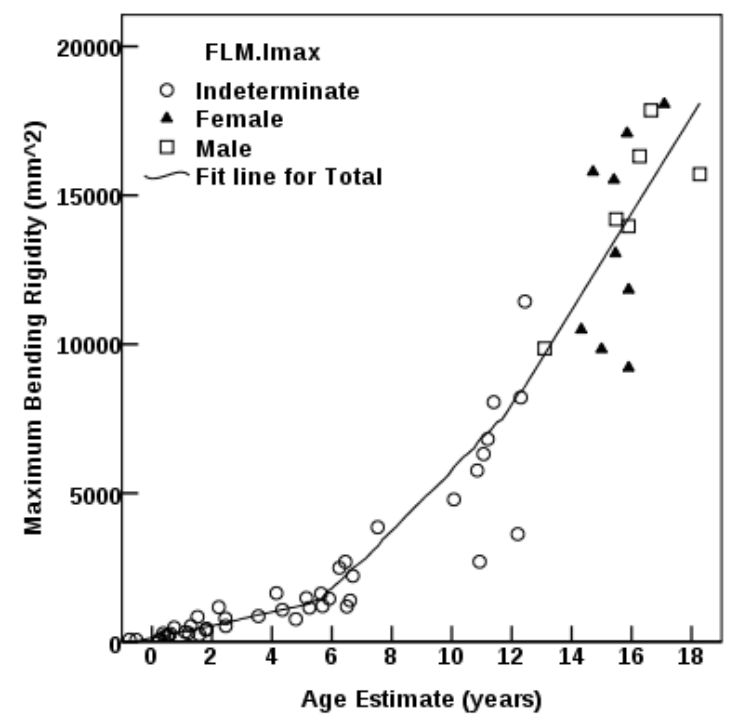

Figure 4.38. Sex estimates for axis of maximal bending strength (Imax) of the left mid-shaft (50\%) femur

In the analyses above, there is no evidence for differences in areas, torsional strength, or shape measures in the left and right femora when left- and right-side growth profiles are evaluated for the sample as a whole. Individual levels of side asymmetry between paired elements are evaluated for the subtrochanteric and mid-shaft femur in Figure 4.39 and Figure 4.40. At both cross-section locations, a level of approximately $10 \%$ asymmetry between torsional strength in the left and right femora is observed throughout development, with no evidence for dominance in either the left or right side. Asymmetry is somewhat higher among the youngest individuals for the subtrochanteric femur, but this is consistent with the higher degree of variation observed for this crosssection in other analyses. The observation of asymmetry levels of approximately $10 \%$ can be attributed primarily to methodological error in assessing limb strength, rather than 
a functional difference in left and right femora. The higher levels of variability in asymmetry observed among the youngest individuals in both growth profiles may be an effect of an absence of significant mechanical constraints on the femur during this period of development.

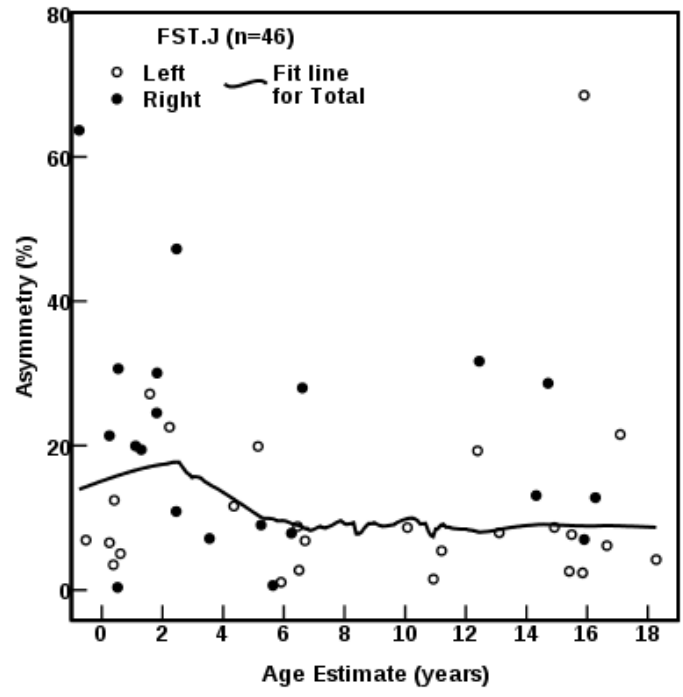

Figure 4.39. Percent asymmetry in torsional rigidity $(J)$ of subtrochanteric $(80 \%)$ femur.

Solid dots indicate a stronger right element, open dotsSolid dots indicate a stronger right element, open dots represent a stronger left element.

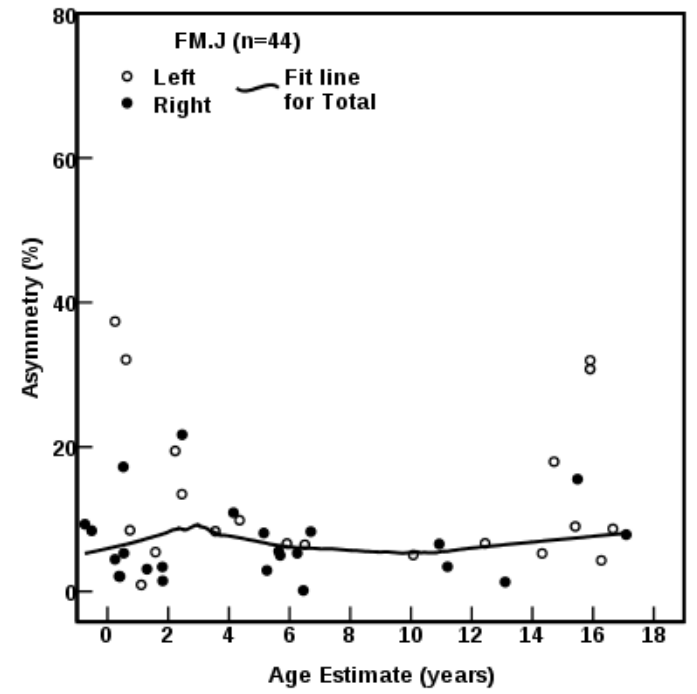

Figure 4.40. Percent asymmetry in torsional rigidity $(J)$ of mid-shaft $(50 \%)$ femur.

Ratios of $\mathrm{I}_{\mathrm{x}} / \mathrm{I}_{\mathrm{y}}$ shape for the tibia reflect a similar pattern of shape change at both the nutrient foramen (Figure 4.41) and mid-shaft (Figure 4.42) cross-section locations. Bending strength along the antero-posterior axis is greater than that of the medio-lateral at birth and continues to decrease, reflecting a changing cross-sectional shape that is expanding along the y-axis. Like the femur, the tibia is therefore adapting to increasing medio-laterally oriented bending loads throughout development. 


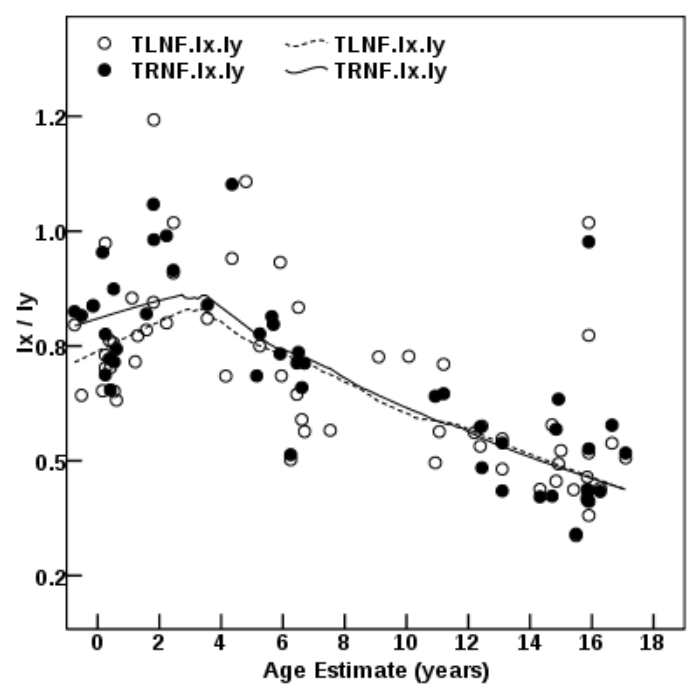

Figure 4.41. Biomechanical shape $\left(I_{x} / I_{y}\right)$ of the nutrient foramen tibia

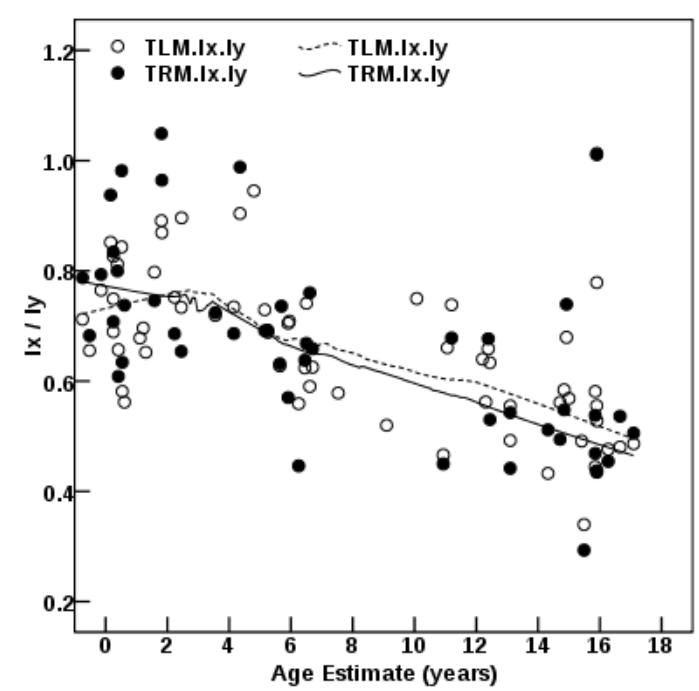

Figure 4.42. Biomechanical shape $\left(I_{x} / I_{y}\right)$ of the mid-shaft $(50 \%)$ tibia

As is observed in the femur, levels of individual asymmetry for the tibiae are relatively low and consistent throughout development, with no evidence of left- or rightside dominance. The nutrient foramen location is more variable and somewhat more asymmetrical than the mid-shaft cross-section, which is not attributed to any side-related functional difference. 


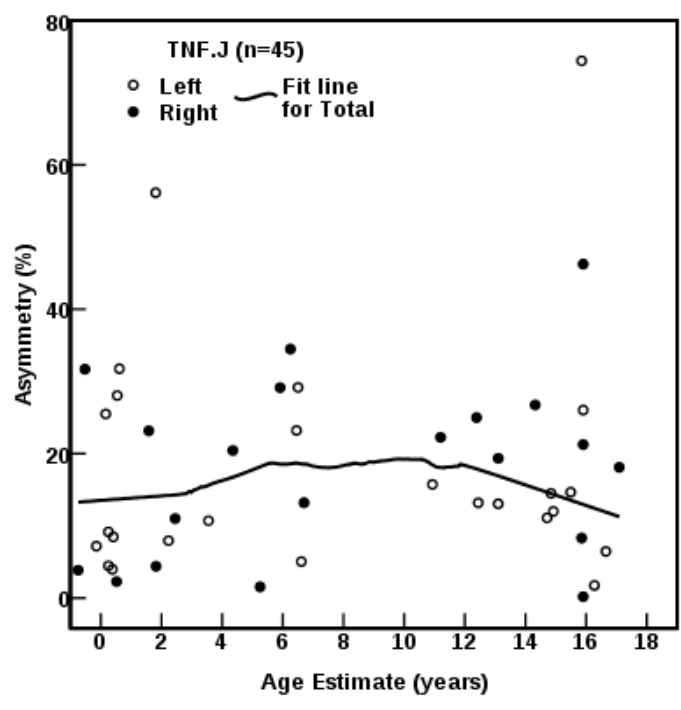

Figure 4.43. Percent asymmetry in torsional rigidity of the nutrient foramen tibia.

Solid dots indicate a stronger right element, open dotsSolid dots indicate a stronger right element, open dots represent a stronger left element.

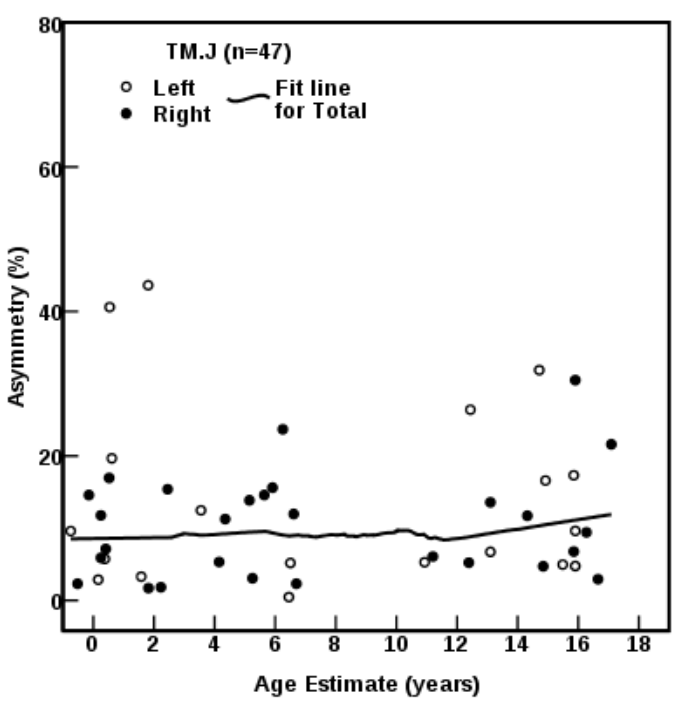

Figure 4.44. Percent asymmetry in torsional rigidity of the mid-shaft $(50 \%)$ tibia.

\subsubsection{Shape and asymmetry in the upper limb}

The skeletal growth profile for biomechanical shape of the mid-shaft clavicle

(Figure 4.45), illustrates that this element is highly variable in its shape throughout ontogeny. The LOESS curve shows a tendency for medio-lateral bending strength to be 1.5 times that in the antero-posterior direction. With respect to the way that the clavicle was oriented in this research, medio-lateral measurements are equivalent to the superoinferior axis when the element is oriented in anatomical position. Growth profiles for the left and right clavicle are equivalent despite the variability in biomechanical shape across the sample. Percent asymmetry values for are approximately $20 \%$ throughout the crosssectional sample (Figure 4.46). There is no evidence that either the left or right clavicle is the dominant side in terms of rigidity. 


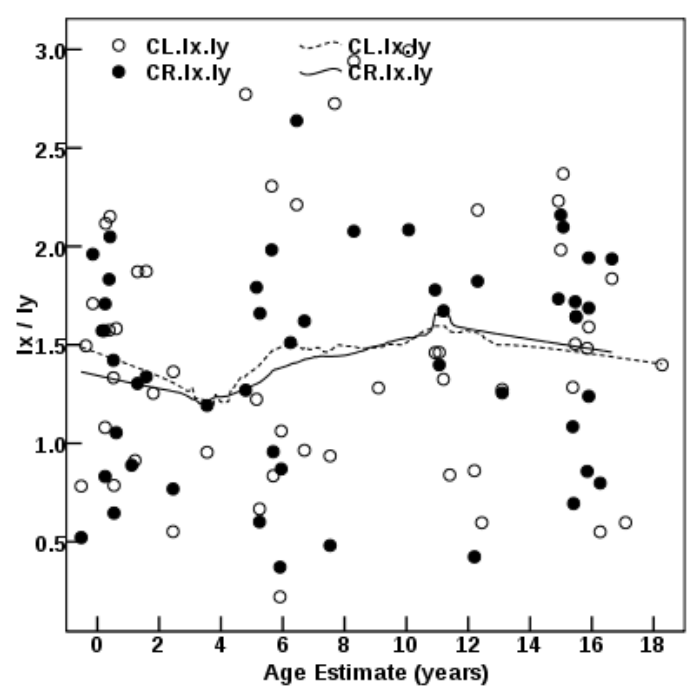

Figure 4.45. Biomechanical shape (Ix/Iy) of the mid-shaft $(50 \%)$ clavicle

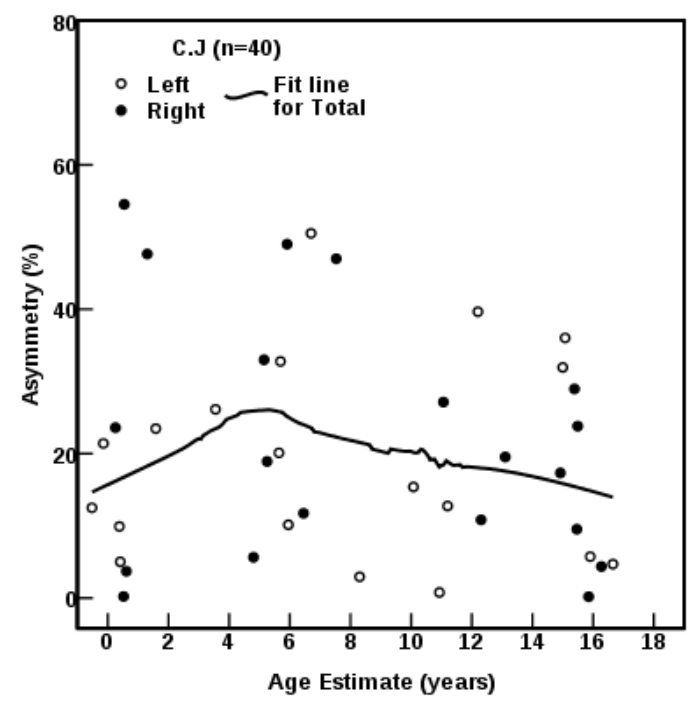

Figure 4.46. Percent asymmetry in torsional strength (J) of the mid-shaft clavicle

Skeletal growth profiles for $\mathrm{I}_{\mathrm{x}} / \mathrm{I}_{\mathrm{y}}$ shape measures of the mid-shaft (Figure 4.47) and distal (Figure 4.48) humerus suggest that the diaphysis is circular in cross-section throughout the growth trajectories. The distal location is, however, highly variable in shape among infants, and tends to be slightly medio-laterally ovoid early in development. Overall, the humeral diaphysis is adapted to resist equal bending forces in the anteroposterior and medio-lateral planes, with no change in the proportions of bending forces encountered throughout growth. There is no difference in biomechanical shape between the left and right humerus in terms proportions of the antero-posterior and medio-lateral bending axes. 


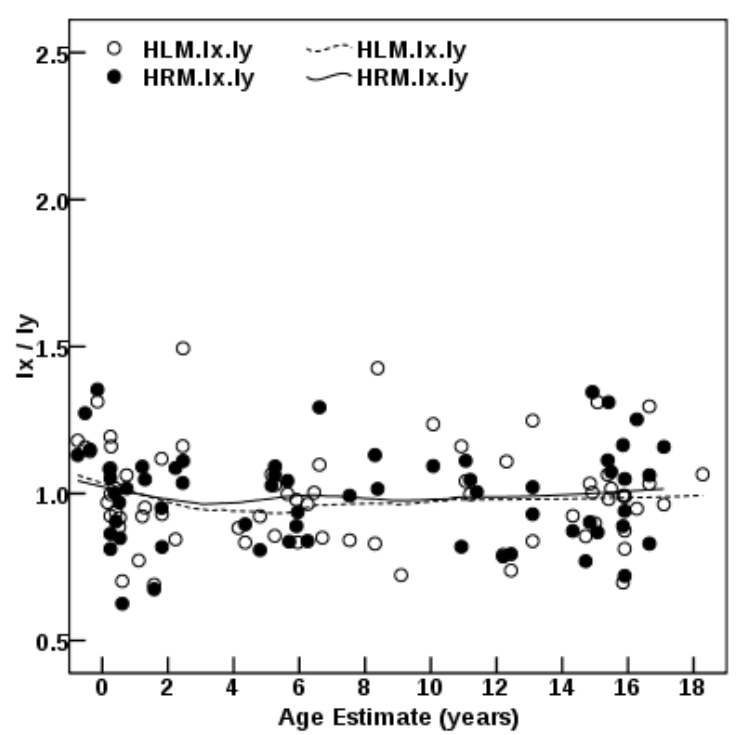

Figure 4.47. Biomechanical shape (Ix/Iy) of the mid-shaft $(50 \%)$ humerus

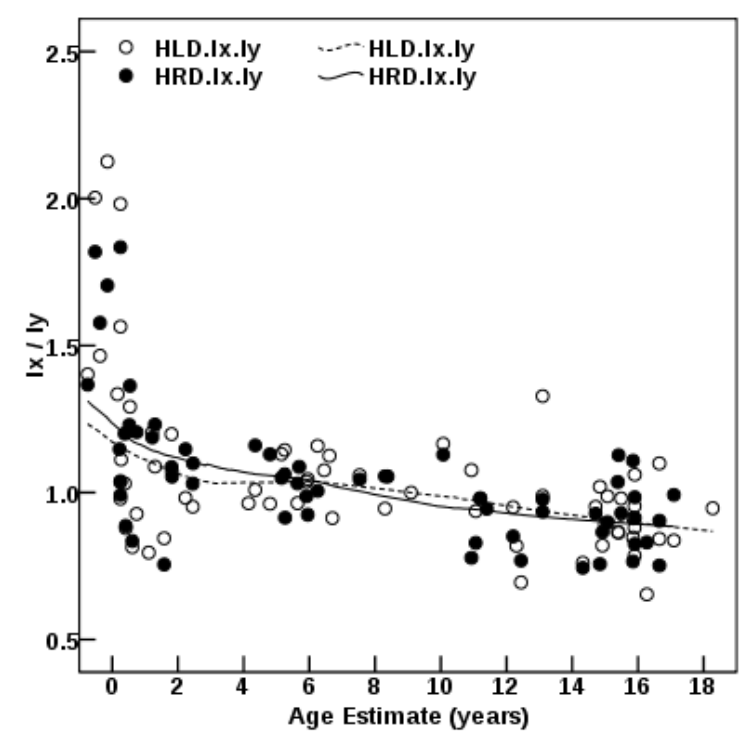

Figure 4.48. Biomechanical shape (Ix/Iy) of the distal $(35 \%)$ humerus

The growth profile for maximal to minimal bending strength of the mid-shaft humerus (Figure 4.49), however, illustrates that there is an appreciable difference in the magnitude of maximal bending rigidity between the left and right humeri. That this side difference was not evident in the growth profile for $\mathrm{I}_{\mathrm{x}} / \mathrm{I}_{\mathrm{y}}$ shape, indicates that the orientation of maximal bending rigidity falls in a plane other than antero-posterior or medio-lateral. The growth profile for the orientation of maximum bending rigidity, theta, (Figure 4.50) demonstrates that the major axis is oriented approximately $30^{\circ}$ from the antero-posterior axis early in development, and as growth proceeds the axis gradually shifts toward the medio-lateral axis. This pattern is mirrored in the left and right elements. 

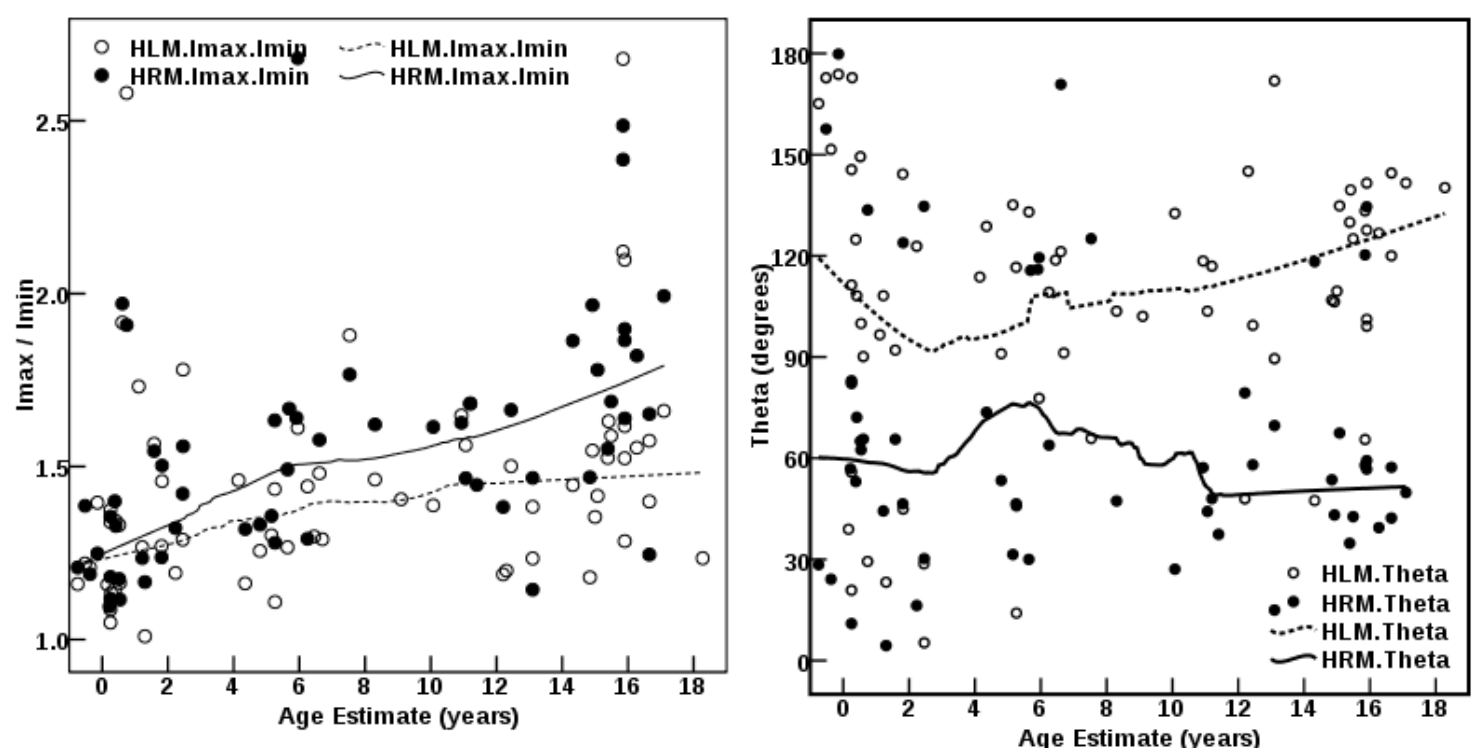

Figure 4.49. Biomechanical shape $\left(I_{\max } / I_{\min }\right)$ of theFigure 4.50. Orientation of maximum bending mid-shaft (50\%) humerus strength (theta) of the mid-shaft $(50 \%)$ humerus

The magnitude of maximal bending rigidity increases relative to the minimum to a greater degree in the right humerus. The divergence in Imax/Imin between limbs is evident in infancy and increases throughout development. Among adolescents, maximum bending rigidity is, on average, 1.75 times that of the minimum for the right humerus, and approximately 1.25 that of the minimum in the left. There is some evidence for a sexbased difference in maximal bending strength. Figure 4.51 is the skeletal growth profile for maximum bending strength of the left mid-shaft humerus. In this plot, many of the female adolescents are below the LOESS curve and all the males are at or above the average maximum bending strength. In the right limb (Figure 4.52), however, male maximum bending strength tends to be higher and there is less overlap with the females. There are three female adolescents with maximal bending strength levels of both the left and right mid-shaft humeri that are in the range of the males, and female arm strength 
across the sample appears to be more symmetrical than that of the adolescent males.

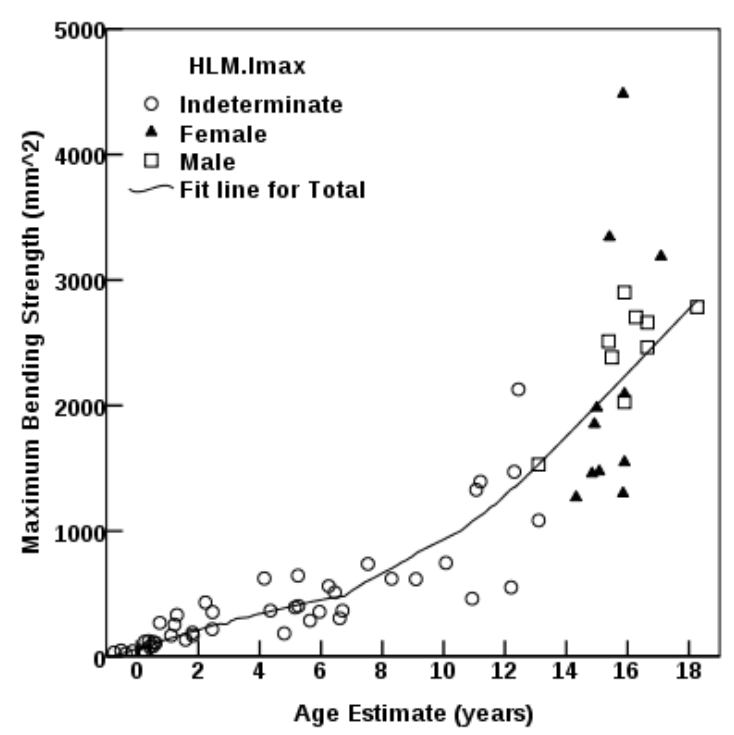

Figure 4.51. Sex estimates for maximum bending strength $\left(I_{\max }\right)$ of the left mid-shaft $(50 \%)$ humerus

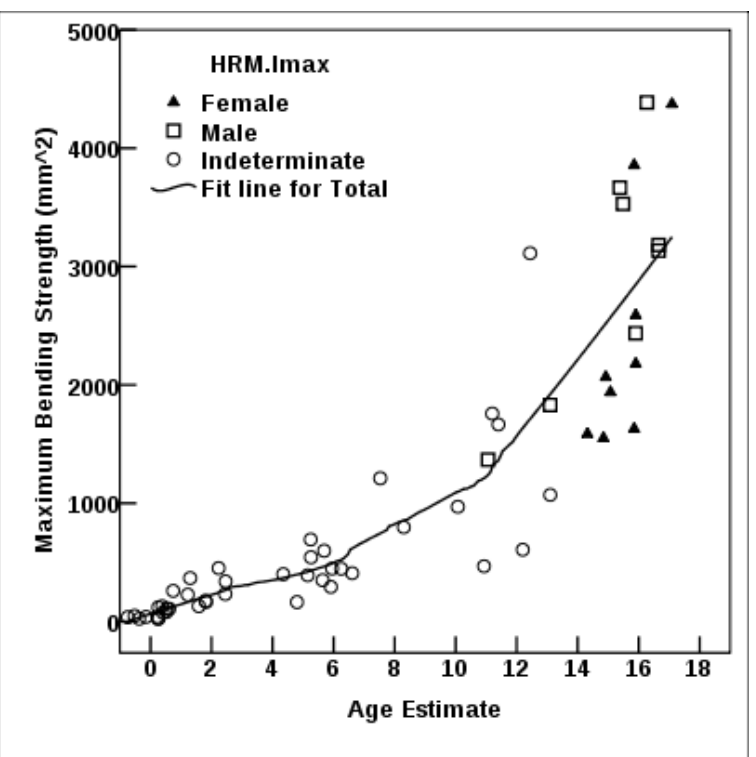

Figure 4.52. Sex estimates for maximum bending strength (Imax) of the mid-shaft $(50 \%)$ humerus

To further investigate asymmetry in humeral strength, individual left-right humerus pairs were evaluated. Torsional strength at the mid-shaft humerus increases in percent asymmetry throughout development, as evidenced by the LOESS curve in the skeletal growth profile (Figure 4.53). Average asymmetry levels increase from approximately $7 \%$ among infants to levels well above $20 \%$ among adolescents. In individuals aged six years and older, the right side tends to be dominant, moreover, is the dominant limb in all cases where percent asymmetry exceeds $10 \%$. Among infants and young children (less than six years of age), the lower levels of asymmetry correspond with no evidence for side dominance.

The distal humeral cross-section location (Figure 4.54) does not show an increase 
in asymmetry over the course of development, with percent asymmetry levels of approximately $15 \%$ throughout growth. There is, however, evidence for right-side dominance among individuals aged six years and older, which is the same pattern observed for side-dominance at the mid-shaft location. In comparing the distal humeral location to the mid-shaft clavicle, it is noted that while both locations have asymmetry levels just under $20 \%$ with no evidence for an age-related increase, the distal humerus does have clear evidence of side dominance.

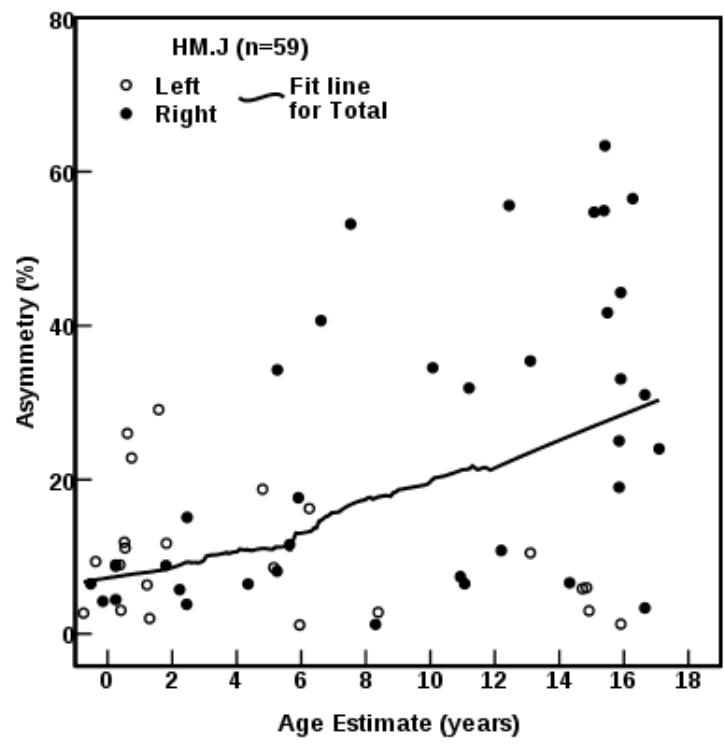

Figure 4.53. Percent asymmetry in torsional rigidity of the mid-shaft $(50 \%)$ humerus Solid dots indicate a stronger right element, open dotsSolid dots indicate a stronger right element, open dots represent a stronger left element.

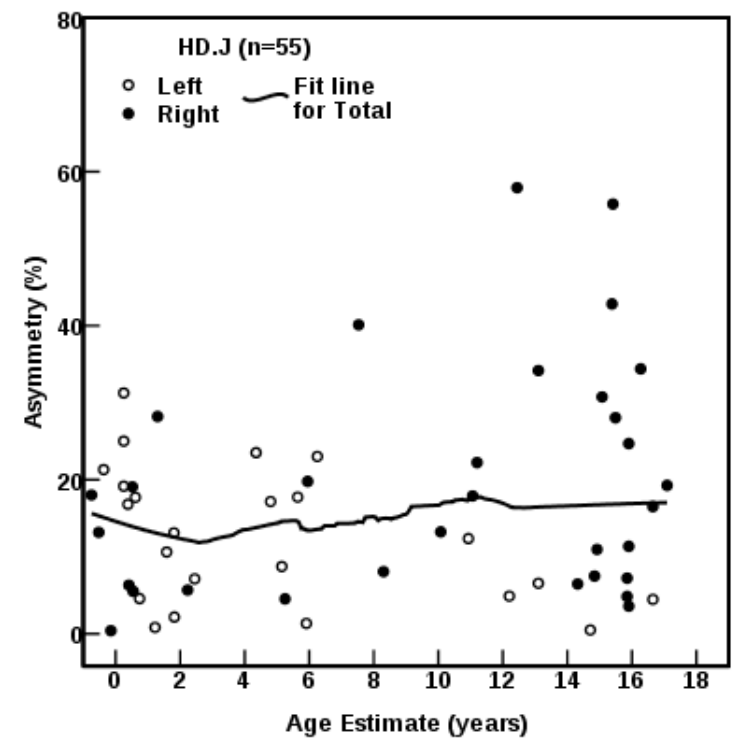

Figure 4.54. Percent asymmetry in torsional rigidity of the distal (35\%) humerus represent a stronger left element.

Side dominance of the mid-shaft humerus is explored further by comparing frequencies of left- and right-side dominant individuals among individuals aged under and over six years; this age was selected based on the pattern of side-dominance observed in Figure 4.53. For mid-shaft humeral cross-sectional properties, including torsional 
strength, total area, and the two shape ratios studied, overall frequencies of left- and right-side dominant individuals do not differ from chance, as indicated by the Chi-square statistics listed in Table 4.2. If the sample is examined by age category, for individuals aged six years and older, right-side dominance is significant with a frequency of $77 \%$ for torsional strength and area properties. For biomechanical shape, the $I_{\max } / I_{\min }$ measure is significantly right-side dominant $(70 \%)$ at the humeral mid-shaft, but the $\mathrm{I}_{\mathrm{x}} / \mathrm{I}_{\mathrm{y}}$ shape measure displays no side dominance. When the sample is evaluated as a whole there is significant right-side dominance in cortical area and in $I_{\max } / I_{\min }$, though as with the other properties there is no side dominance among individuals aged six years and under.

\begin{tabular}{|c|c|c|c|c|c|c|c|c|c|c|c|c|}
\hline & \multicolumn{4}{|c|}{$\begin{array}{c}\text { All } \\
(\mathbf{n}=59)\end{array}$} & \multicolumn{4}{|c|}{$\begin{array}{c}\text { Under } 6 \text { years } \\
\quad(n=29)\end{array}$} & \multicolumn{4}{|c|}{$\begin{array}{c}\text { Over } 6 \text { years } \\
(n=30)\end{array}$} \\
\hline & Left & Right & $X^{2}$ & $p$ & Left & Right & $X^{2}$ & $p$ & Left & Right & $X^{2}$ & $p$ \\
\hline$\underline{\text { HM.J }}$ & $39 \%$ & $61 \%$ & 2.864 & 0.091 & $55 \%$ & $45 \%$ & 0.310 & 0.577 & $23 \%$ & $77 \%$ & 8.533 & 0.003 \\
\hline HM.TA & $39 \%$ & $61 \%$ & 2.864 & 0.091 & $55 \%$ & $45 \%$ & 0.310 & 0.577 & $23 \%$ & $77 \%$ & 8.533 & 0.003 \\
\hline HM.CA & $34 \%$ & $66 \%$ & 6.119 & 0.013 & $45 \%$ & $55 \%$ & 0.310 & 0.577 & $23 \%$ & $77 \%$ & 8.533 & 0.003 \\
\hline HM.Ix/Iy & $53 \%$ & $47 \%$ & 0.15 & 0.696 & $59 \%$ & $41 \%$ & 0.862 & 0.353 & $47 \%$ & $53 \%$ & 0.133 & 0.715 \\
\hline HM.Imax/Imin* & $35 \%$ & $65 \%$ & 5.255 & 0.022 & $39 \%$ & $61 \%$ & 1.286 & 0.257 & $30 \%$ & $70 \%$ & 4.481 & 0.034 \\
\hline
\end{tabular}

Table 4.2. Chi-square statistics for side dominance in the mid-shaft humerus $*_{\mathrm{n}}=55 / 28 / 27$, for All, Under 6, and Over 6, respectively

Sex estimates for torsional strength asymmetry at the mid-shaft humerus more clearly demonstrate a sex difference than was evident in the above comparison of maximum bending strength in the left and right humeri. Most females fall at or below the LOESS curve for asymmetry while most males are at or above average the curve. There are two females with highly asymmetrical mid-shaft humeral torsional strength, and one male adolescent with very symmetrical humeri. 


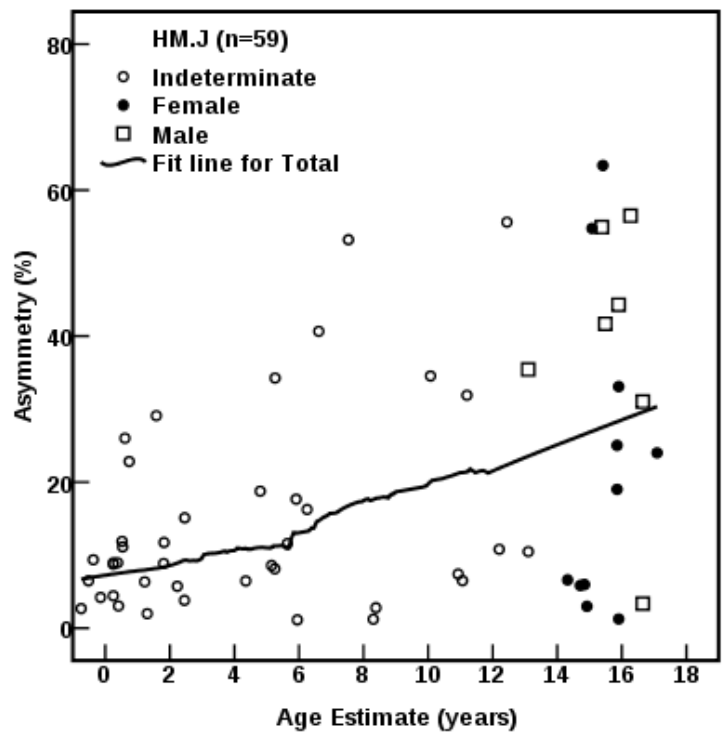

Figure 4.55. Percent asymmetry in torsional rigidity of the mid-shaft $(50 \%)$ humerus by sex
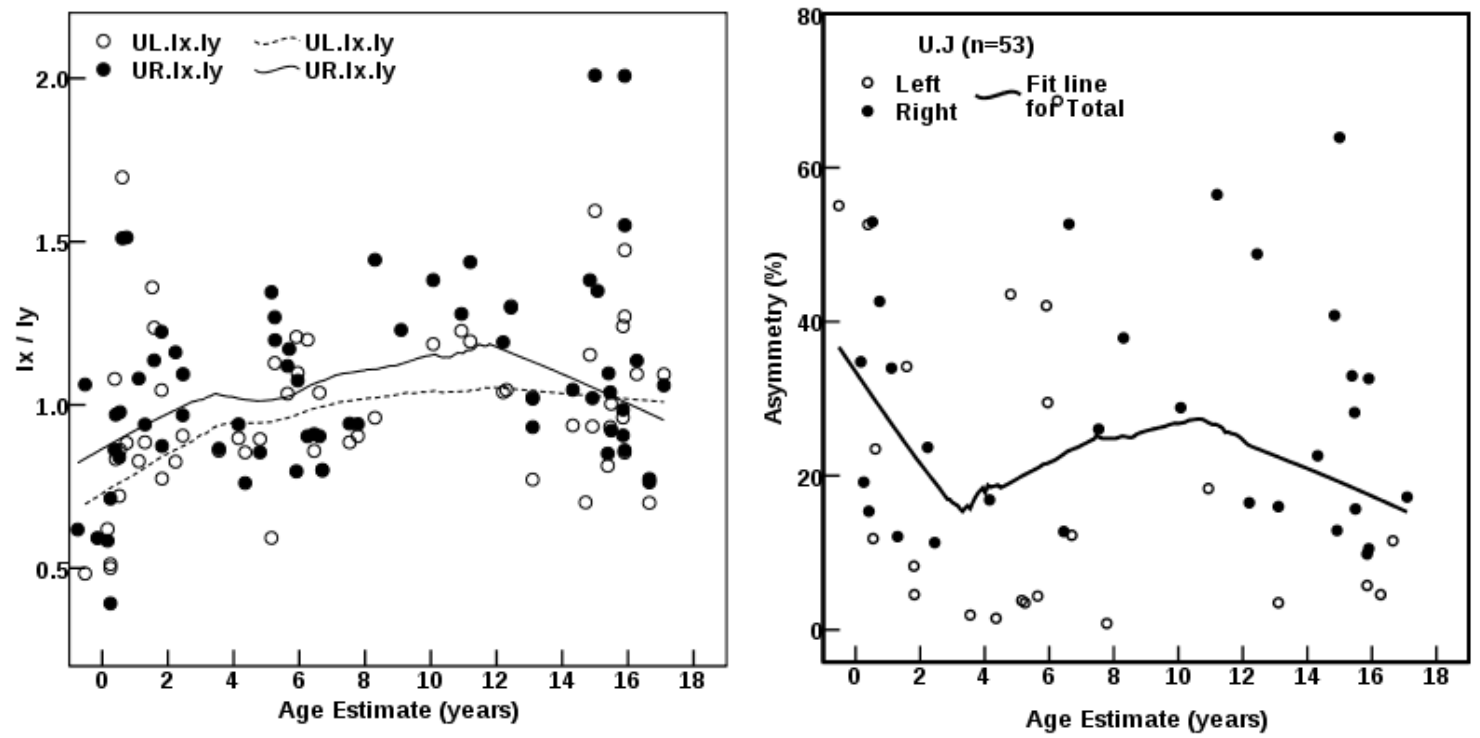

Figure 4.56. Biomechanical shape (Ix/Iy) of the mid-shaft $(50 \%)$ ulna

Figure 4.57. Percent asymmetry in torsional rigidity of the mid-shaft $(50 \%)$ ulna.

Solid dots indicate a stronger right element, open dots represent a stronger left element. 
Biomechanical $\left(\mathrm{I}_{\mathrm{x}} / \mathrm{I}_{\mathrm{y}}\right)$ shape of the mid-shaft ulna is proportional throughout the developmental trajectory (Figure 4.56). The LOESS curves suggest that the left limb is more antero-posteriorly ovoid than the right side, except among the oldest adolescents. Age does not appear to explain the variation observed in $\mathrm{I}_{\mathrm{x}} / \mathrm{I}_{\mathrm{y}}$ shape. Analysis of asymmetry in torsional rigidity of the mid-shaft ulna shows that the distal arm limb segment follows the pattern observed for the distal humerus. There is no evidence that asymmetry increases with age, in fact, the LOESS curve illustrates significant variation throughout the course of development (Figure 4.57). There is, however, evidence for right-limb dominance in ulnar torsional strength among individuals aged six years and older. As with the humerus, older individuals with dominant left elements are those with very low levels of asymmetry.

\subsubsection{Summary of biomechanical shape and asymmetry}

In the mid-shaft and subtrochanteric femur, shape is highly variable among individuals aged under two years. Throughout early childhood, the femoral diaphysis tends to be medio-laterally ovoid but expands in the antero-posterior axis with growth, reflecting increased bending strength in the medio-lateral plane. Maximal bending strength increases throughout the growth trajectory, to a level 1.5 times greater than minimum, on average, among adolescents. Measurement of theta illustrates that the orientation of maximum bending strength moves toward the antero-posterior axis with increasing age, confirming the observation of changes in $\mathrm{I}_{\mathrm{x}} / \mathrm{I}_{\mathrm{y}}$ shape throughout development. There is no evidence for sex-based differences in magnitude of maximum 
bending strength at the mid-shaft femur among adolescents. Levels of asymmetry in the femur are about $10 \%$, with no evidence of side dominance; this level of asymmetry likely reflects combined random and measurement error. The tibia shows the same patterns of biomechanical ontogenetic shape change.

As with the lower limb, there is a high level of variability in biomechanical shape in the elements of the upper limb among individuals aged under two years. Ratios of $\mathrm{I}_{\mathrm{x}} / \mathrm{I}_{\mathrm{y}}$ suggest that the clavicle and ulna are variable in shape throughout the course of development, while the humerus is comparatively proportional in bending strength along the $\mathrm{x}$ - and $\mathrm{y}$ - axes. Analysis of Imax for the mid-shaft humerus illustrates that the principle axis shifts throughout development from approximately $60^{\circ}$ toward the mediolateral axis. While the orientation of the principle axis is mirrored in both the left and right humeri, the magnitude of Imax is greater in the right humerus. Asymmetry levels in the mid-shaft humerus are well over $20 \%$ among some of the oldest individuals in the sample, while the distal humerus and the clavicle and ulna have asymmetry levels that are generally under $20 \%$. The right element is the dominant side in analyses of left-right pairs in the distal humerus and ulna, even though percent asymmetry levels are lower than the mid-shaft humerus at these locations. No side dominance is evident in the clavicle. At the mid-shaft humerus, more than $70 \%$ of individuals show right-side dominance for areas, torsional rigidity and Imax/Imin among individuals aged over six years. Males tend to have more asymmetrical torsional mid-shaft humeral rigidity than females among adolescents. 


\subsection{Body-size standardization of cross-sectional properties}

\subsubsection{Determinants of limb strength}

In order to explore the relationship between body size-related variables and biomechanical characteristics of long bones, linear regression was used to model crosssectional properties of the mid-shaft femoral and humeral diaphysis in terms of estimated estimated body mass and element length. These independent variables were selected as factors that are likely to relate closely to diaphyseal size and strength given the functional influence of weight-bearing in skeletal robusticity. The goal of the analysis was to determine whether torsional rigidity $(\mathrm{J})$, bending rigidity $(\mathrm{Imax})$, and total area (TA) of the humerus and femur relate uniquely to body size during ontogeny, by evaluating the relative contributions of mass and limb length to the variation observed in cross-sectional properties throughout growth.

Simple linear regression was performed for each cross-sectional property separately on each of age, mass, and diaphysis length (femur or humerus). Secondly, each cross-sectional property was modelled with the three independent variables (age, mass, and length) using an entry criterion of $p<0.05$. Each model was tested for collinearity among the independent variables. These analyses were performed on both the left and right elements, but given the similarity of the results between sides, coefficients of determination for each model are reported in Table 4.3 for the left side only (femur, left $n=57$, right $n=44$; humerus left $n=63$, right $n=58$ ); all models are significant $(p<0.01)$. The residuals for mass on age, and length on age were included as 
independent variables to model the residuals from each cross-sectional property regressed on age (Table 4.3). This approach controls for age while evaluating the relative contribution of mass and element length as predictors of each biomechanical property.

\begin{tabular}{l|c|c|c|c|c|c|c}
\hline & \multicolumn{3}{|c|}{ Simple Models $\left(\mathbf{r}^{2}\right)$} & \multicolumn{4}{c}{ Complete Model $\left(\mathbf{r}^{2}\right)$} \\
\cline { 2 - 8 } & Age & Mass & Length & Overall & Partial Age & Partial Mass & Partial Length \\
\hline FL.TA & 0.945 & 0.916 & 0.940 & 0.955 & 0.365 & 0.289 & 0.208 \\
\hline FL. Imax & 0.852 & 0.858 & 0.809 & 0.886 & 0.393 & 0.488 & -0.300 \\
\hline FL.J & 0.856 & 0.855 & 0.813 & 0.885 & 0.397 & 0.453 & -0.276 \\
\hline HL.TA & 0.889 & 0.871 & 0.917 & 0.927 & 0.129 & 0.273 & 0.240 \\
\hline HL. Imax & 0.764 & 0.764 & 0.818 & 0.864 & 0.168 & 0.432 & -0.100 \\
\hline HL.J & 0.799 & 0.805 & 0.823 & 0.874 & 0.210 & 0.452 & -0.143 \\
\hline
\end{tabular}

Table 4.3. Correlation coefficients for linear models of body-size related determinants of biomechanical properties

None of the independent variables analysed can be singled out as the strongest determinant of cross-sectional size or strength. Each of the complete models, which include all three independent variables, have significant multi-collinearity between estimated age, estimated mass, and diaphysis length. In evaluating the relative predictive value of the multivariate models over the simple linear regression models, the complete model is considered a better predictor if the coefficient of determination exceeds that of the best simple regression model by at least 0.05 . Adding variables to a model will increase the coefficient of determination to some degree, but it is important to evaluate whether the addition of factors to a model does so meaningfully. None of the complete models improved upon the best simple regression model by more than the $5 \%$ criterion.

In examining the simple regression models, each of the independent variables 
explains a similar proportion of the variability in cross-sectional properties, with coefficients of determination ranging between $0.764-0.945$ for estimated age, $0.855-$ 0.916 for estimated mass, and $0.809-0.940$ for diaphyseal length. More variance in total area is explained by these determinants than is for the strength properties (bending or torsional strength), suggesting more complexity in the relationship between body-size related variables and biomechanical properties, than between body-size related variables and diaphyseal area. In the humerus, diaphysis length consistently explains a greater proportion of variance in cross-sectional properties than does estimated age or mass, and in the femur, estimated age explains a greater proportion of variance in total area and strength properties if the coefficient for the right element is substituted for the left in the case of maximum bending strength. The main result of these analyses is that less than approximately $10 \%$ of variation remains unaccounted for in evaluating the relationship between total area and the independent variables, whereas approximately $20 \%$ of variation remains unaccounted for in modelling estimated age, mass, and diaphysis length as determinants of strength properties (Imax and $\mathrm{J}$ ).

\subsubsection{Size-standardized cross-sectional properties}

Skeletal growth profiles for size-standardized cross-sectional properties illustrate that very little variability remains throughout the entire course of growth once body size is accounted for. Area measurements are standardized by an estimate of body mass, as determined by the mass estimation formulae for juveniles derived from the Denver Growth Study data (described above). Second and polar moments of area are 
standardized by the product of mass and lever arm length (element diaphyseal length).

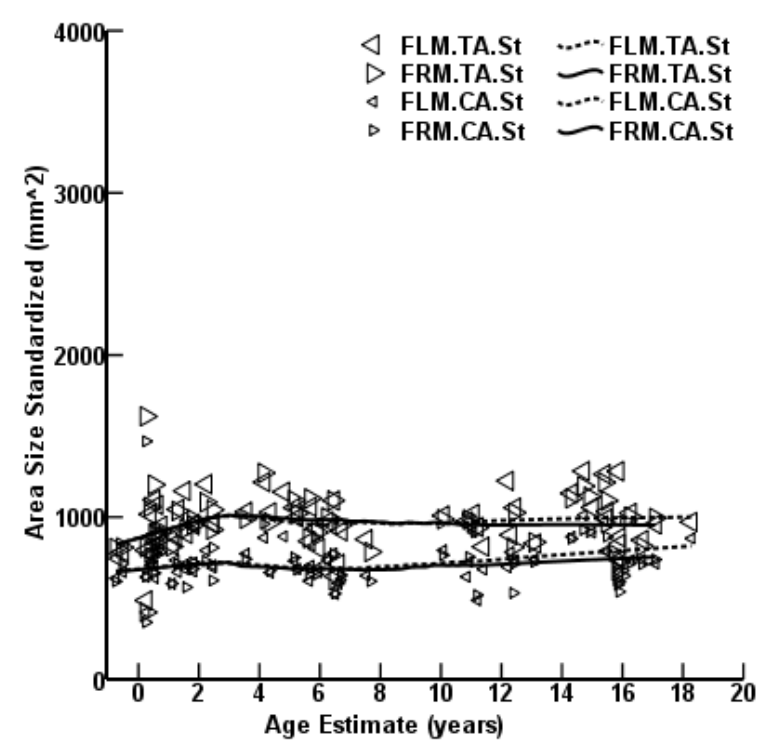

Figure 4.58. Size-standardized areas of the midshaft $(50 \%)$ femur

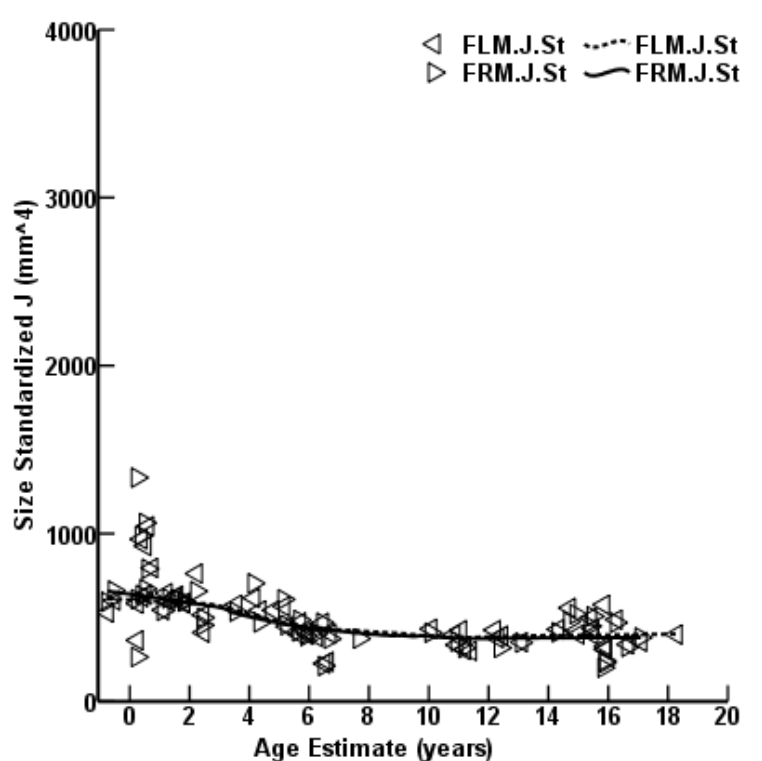

Figure 4.59. Size-standardized torsional rigidity (J) of the mid-shaft $(50 \%)$ femur

The LOESS curves for areas (Figure 4.58) and torsional rigidity (Figure 4.56) for the mid-shaft femur illustrate that there is very little age-related variation in crosssectional properties when body-size is controlled for. Among infants, variability in sizestandardized measures is somewhat greater; if it is assumed that measurement error is not age-dependent, the variation observed can be attributed to an absence of mass-related forces (axial and torsional) acting on the femur during infancy. 

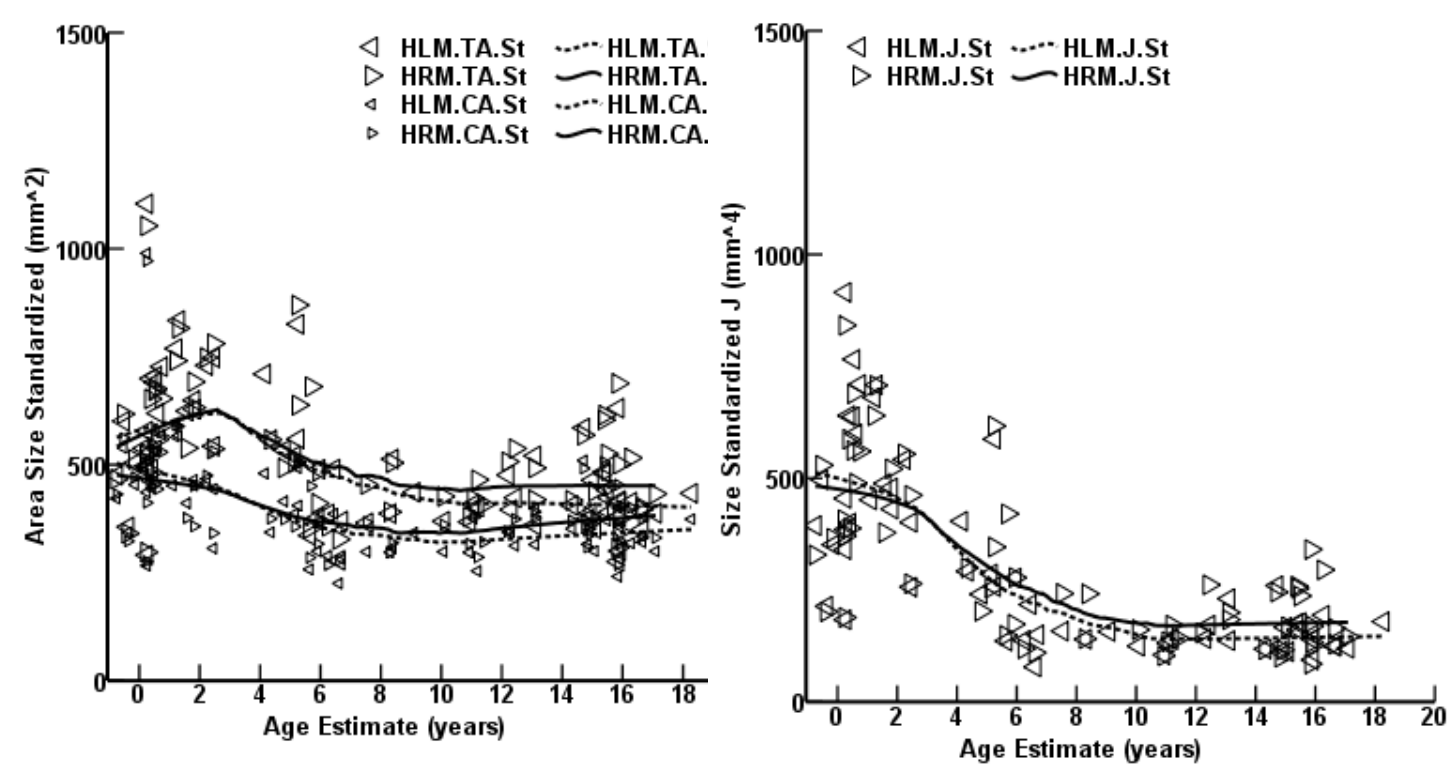

Figure 4.60. Size-standardized areas of the mid-Figure 4.61. Size-standardized torsional rigidity (J) of $(\mathbf{5 0} \%)$ humerus the mid-shaft $(50 \%)$ humerus

Size-standardized areas (Figure 4.60) and torsional rigidity (Figure 4.61) for the mid-shaft humerus are less-well controlled for by body size than was the femur. Variation is greatest among individuals aged six years and younger; among older individuals, body size seems to control for cross-sectional properties as both areas and the polar second moment of area are stable throughout the remainder of the growth trajectory. The patterns observed in the mid-shaft femur and humerus are also observed in the remaining cross-sectional locations studied (Appendix E). 


\section{Chapter 5}

\section{Discussion and Conclusions}

The skeletal growth profiles for areas and polar second moments of areas at all sixteen skeletal sites analysed illustrate that much of the variability in cross-sectional geometric properties of diaphyses is age-related. The demonstration of this basic assumption, that diaphyseal strength varies predictably with age in an ontogenetic sample, is a necessary pre-requisite to any further analysis of the patterning and timing of developmental trajectories for appositional growth. Curve-fitting analysis demonstrates that approximately $90 \%$ of the variation in total area, cortical area, and torsional rigidity can be explained by age when fit to a cubic curvilinear model. Locally-weighted regression provides skeletal growth profiles that reflect variation specific to age ranges within the trajectories, demonstrating changes in growth tempo throughout development.

Analyses of linear growth, which evaluate the development of stature and body proportions, are well established as a means through which questions about the health status and morphological adaptability of past populations can be explored. The study of appositional growth in juveniles, however, is an emerging research theme, and so there is value in describing some of the basic patterns observed with respect to the ontogeny of bone functional adaptation in a well-preserved archaeological modern human skeletal sample, before attempting to interpret the data in the context of specific behavioural 
hypotheses.

Total, cortical, and medullary area measurements increase in a pattern that appears linear from the LOESS profiles, in contrast to trajectories for polar second moments of area, which show an sharp increase in torsional rigidity measures during mid-childhood. This observation reflects the significance of the distribution of tissue about the diaphysis in imparting resistance to loading: cortical tissue located further from the linear axis of the long bone contributes proportionally more to strength. The exponential pattern of torsional strength increase is a function of the measurement units for polar second moments of area $\left(\mathrm{mm}^{4}\right)$ which are multiplied by the squared distance from the section centroid. So while total area $\left(\mathrm{mm}^{2}\right)$ increases in a linear fashion, the effect of multiplying area units of cortical tissue by distance has the effect of increasing polar second moments of area in an exponential fashion as the diaphyseal circumference expands.

The confidence with which the general patterning of the trajectories for Later Stone Age juveniles can be described is limited by the paucity of individuals aged between eight and ten years. Under-representation in the sample age distribution for individuals in late childhood may have the effect of exaggerating the differences observed in the trajectory in infancy and childhood, which shows gradual increase in bone strength relative to adolescence when strength increases are greater. It is worth noting that the remains of older children are rare in archaeological skeletal assemblages because this developmental phase is typically one of lower risk to life relative to the 
susceptibility of infants and young children to the stresses of infection and weaning, and the acute danger that accompanies the risk taking behaviour incumbent to human adolescence; the Later Stone Age sample is, therefore, typical of the age distribution among juveniles characteristic of archaeological skeletal assemblages.

The remainder of this chapter is organized around two behavioural themes, in the context of existing observations of Later Stone Age adults and ethnographicallydocumented child behaviour in hunter-gatherer societies. Mobility characteristics of Later Stone Age juveniles are explored in terms of the changing locomotory regimes that accompany early development and the physical activity levels that characterize the subsistence strategies employed by this population. Upper limb morphology is interpreted in relation to archaeological evidence for the types of tools used by Later Stone Age hunter-gatherers and expectations for the role of children in the social structure, with respect to when children begin to engage in gender-based subsistence activities.

\subsection{Developing locomotory behaviours and mobility levels}

The morphology of the lower limb is influenced by the loading demands of body mass, with the nature of the strain borne by the lower limb being a product of the specific locomotory regime in terms of the relative contribution of axial/compressive and bending/torsional forces placed on the skeletal elements. The cross-sectional geometric properties of the femur and tibia present a clear pattern for age-related changes in the 
loading of the lower limb of hunter-gatherer juveniles. There are several lines of evidence to suggest that young children are walking well-before two years of age.

Before considering the relevant data, it is important to account for two factors when interpreting skeletal growth profiles in terms of the timing of behavioural change. The age at death estimates that correlate with observed changes in strength measures reflect behavioural patterns that have been established for a period of sufficient duration that they can be manifest in the skeleton. Secondly, the children comprising skeletal growth profiles based on archaeological assemblages are necessarily those members of the juvenile population who died. The assumption made in this research is that the juveniles studied were not significantly more frail than other members of their cohort, and are therefore representative of "normal" Later Stone Age juveniles with respect to health status. This assumption is, in part, supported by selecting juveniles for study who show no skeletal signs of chronic disease that may have had an impact on growth or behaviour prior to death. While cause of death remains unknown, the circumstances surrounding death are assumed to be acute in nature (Harrington \& Pfeiffer, 2008; Pfeiffer, 2007).

Areas and polar second moments of area for both the proximal and distal crosssectional sites studied in the femur and tibia show a gradual increase between from infancy to approximately six years of age, reflecting adaptation to increasing axial/compressive and torsional loads. When torsional strength in the lower limb 
elements are evaluated relative to the humerus, an increase in leg strength is evident that spans the period from two to six years. In individuals aged two years and under, humeral to femoral/tibial strength is proportional, after which leg strength increases relative to the humerus until age six. This pattern reflects the morphological response to a change in the locomotory regime that affects the lower limb only, which is interpreted as the onset of walking. After age six, leg to arm strength proportions cease to increase further, which must reflect a relative increase in arm strength given that all limbs continue to gain strength throughout development.

Measures of biomechanical shape provide additional evidence for a functional response to walking during early childhood. The mid-shaft femoral diaphysis expands in the antero-posterior axis, reflecting an adaptation to resist bending forces in the mediolateral plane. The orientation of maximal bending resistance also begins to shift toward the antero-posterior axis. These biomechanical characteristics are consistent with the development of the femoral pilaster which is an anatomical feature which is expressed variably in relation to robusticity in the adult femur.

Based on the skeletal growth profiles alone, the developmental timing of walking cannot be identified with precision; the skeletal morphology suggests only that a change to the locomotory regime of young children is manifest around two years of age. Modern Western children typically progress through a series of stages where independent walking is achieved by approximately sixteen months. Precocious initiation of walking has been 
reported among Kalahari Khoesan children (Konner, 1976), and has been attributed to the particular view of Khoesan parents about the needs of infants with respect to motor development. Konner reports a belief among Khoesan caregivers that infants will fail to progress through the stages of sitting, standing, and walking unless actively taught to do so. Khoesan adults were observed to assist infants in sitting upright by using sand to build a supportive "seat" in which the child could sit unassisted, or are generally held upright rather than being placed laying flat as is often the way Western societies care for infants. Konner suggests that consistent stimulation of the stepping reflex by encouraging the infant to use her legs and feet by engaging with the caregiver's lap or the ground, may be a factor in supporting waking at an earlier age than is typical of Western children.

Skeletal growth profiles for lower limb cross-sectional properties illustrate increasing variability in leg strength measures among adolescents. While there is an overall pattern of increasing measures of axial/compressive and torsional strength throughout development, and biomechanical shape measures provide evidence for the development of the femoral pilaster, the magnitude of lower limb strength varies widely from late childhood onward. This feature of the growth trajectory stands in contrast to the relatively low levels of variance observed in the lower limb following the onset of walking among young children. With the onset of puberty, body mass variance is also likely to increase as are differences in the behaviours engaged in by adolescent males and females. As the lower limb morphology is influenced by both body mass and behavioural 
effects, it may be difficult to interpret the relative effects of these factors. Body-size standardized areas and torsional strength measures for the lower limb illustrate equal (low) variance throughout the growth trajectory, in contrast to the unstandardized measures. This provides support for a behavioural interpretation of the increased variability observed among adolescents. Sex estimates among the adolescents in the sample illustrate significant overlap between strength measures for males and females, so while levels of loading vary considerably among older juveniles, this effect cannot be attributed to a clear distinction in the levels of physical activity engaged in by male and females adolescent Later Stone Age hunter-gatherers.

\subsection{Subsistence behaviours reflected in upper limb morphology}

The upper limb provides an opportunity to explore localized mechanical effects through comparison of the left and right elements, which are subject to the same systemic influences on appositional growth. Comparisons of strength measures in the left and right limbs illustrate right side dominance for the humerus and ulna, but interestingly, not

for the clavicle. Cross-sectional properties for the clavicle showed much more variability than the other elements studied. Among the skeletal growth profiles for unstandardized areas and polar second moments of areas, average strength values for the left and right humerus and ulna begin to diverge during late childhood, and continue to do so throughout the rest of development. Right elements have greater strength measures than left, across the sample as a whole. Analysis of individual left-right upper limb element pairs brings additional resolution to the features of asymmetry and side dominance 
observed among Later Stone Age hunter-gatherers.

While humeral strength increases throughout growth, as evidenced by the growth profiles for areas and torsional strength, analysis of proportional strength in the humerus relative to the femur and tibia illustrates that significant gains in upper arm strength occur beginning in late childhood. This feature of growth is accompanied by the onset of dominance of the right upper limb. Measures of biomechanical shape, however, show that humeri are symmetrical with respect to the orientation of maximum bending strength, which shifts toward the medio-lateral axis from an orientation of approximately 60 degrees at the beginning of development. It is the magnitude, rather than the nature of loading that differs between individual left-right humerus pairs.

Upper limb asymmetry is most evident at the mid-shaft humerus, with levels in the range of $30 \%$ noted among the most asymmetric adolescents. This level of asymmetry is consistent with levels noted for Later Stone Age hunter-gatherer adults (Stock, 2002a), although is at the high end of the range reported among a range of modern human archaeological samples (Ruff, 2008). Specialized loading such as that engaged in by professional racquet sport players reported in the classic study of Jones and colleagues (1977) has produced the highest reported values for upper limb asymmetry which are in the range of $40 \%$.

Asymmetry levels at the other upper limb cross-sectional locations were appreciably lower, as stated above the clavicle was not asymmetrical, while the distal 
humerus and mid-shaft ulna had levels of asymmetry just above the levels reported for the lower limb. It would seem that an average of $10 \%$ asymmetry constituted the the range for measurement error in this study, as this is the approximate level observed in the lower limb. Side dominance analysis produced patterns that provide additional support for the developmental patterns in laterality observed in Later Stone Age juveniles. While asymmetry levels are relatively low for the distal humerus and ulna, these section locations show consistent right-side dominance, in keeping with the observations for the mid-shaft humerus. Side dominance for all cross-sectional properties of the mid-shaft humerus is apparent only among individuals aged six years and older, with over $70 \%$ of individuals displaying right-side dominance.

Evidence for sexual dimorphism during adolescence is stronger in the humerus than in the lower limb with respect to mobility levels. Torsional strength values in the humerus overlap to a lesser degree among males and females, with most males having values at or above the mean. These results suggest that Later Stone Age juveniles are beginning to engage in gender-specific behaviours during adolescence or perhaps late childhood, which are creating the adaptive response observed in the skeletal growth profiles. Stock and Pfeiffer $(2001,2004)$ attribute upper limb symmetry in females to the use of the digging stick. The toolkit of Later Stone Age males included the spear and the light-draw bow, both of which are likely to be used with a preferred limb, which among most individuals would be the right side as indicated in the ontogenetic data presented in this research which is in keeping with the patterns observed among the adult subset of the 
Later Stone Age sample (Stock, 2002a).

\subsection{Conclusions}

This research set out to explore developmental patterns in appositional growth among Later Stone Age hunter-gatherers. Analysis of cross-sectional geometry in the postcranial elements of sub-adults produced measures of bone strength that vary predictably with age, making it possible to analyze skeletal growth profiles for robusticity. Later Stone Age juveniles achieve bone strength values comparable to the average for adults of the same population by late adolescence. Sexual dimorphism in the patterns of relative leg to arm strength that suggest higher levels of mobility among males, and in values for upper limb asymmetry that differentiate adult males from females and juveniles, have not yet fully developed by late adolescence. Indeed, the skeletal growth profiles presented here show no evidence for a cessation of appositional growth among the oldest sub-adults included in the sample.

The developmental timing of behaviours cannot be determined with precision from skeletal growth profiles based on a cross-sectional sample of children. The interval between habitual loading and its manifestation in the skeleton is another limit to the ability to estimate the timing of the onset of behaviours that initiate functional adaptations in the skeleton. Nevertheless, the patterning in strength measures observed reflects the changing locomotory behaviours of early childhood, with the onset of walking evidenced by a significant increase in relative lower to upper limb strength after 
two years of age. Later Stone Age children may have received the type of care that was observed among Kalahari Khoesan peoples, in which caregivers took an active role in encouraging motor development among infants. Older male children may have been engaging in the physical activities that reflect the hunting activities of adult males, as reflected patterns of humeral asymmetry that are consistent with the use of spears and light draw bows. Evidence for significant differences in the levels of mobility among male and female children is not evident to the degree observed between male and female Later Stone Age adults. It would seem that trekking behaviours among juvenile males and females was similar, as older male children and adolescents may not have participated in the larger scale hunting journeys away from camp that characterize adult male subsistence. 


\section{Bibliography}

Adams, P., \& Berridge, F. (1969). Effects of Kwarshiorkor on cortical and trabecular bone. Archives of Disease in Childhood, 44, 705-709.

Armelagos, G. J., Mielke, J. H., Owen, K. H., Van Gerven, D. P., Dewey, J. R., \& Mahler, P. E. (1972). Bone growth and development in prehistoric populations from Sudanese Nubia, Journal of Human Evolution, 1(1), 89-119.

Barr, D., Prader, A., \& Shmerlin, D. (1972). Catch-up growth in malnutrition, studied in celiac disease after institution of a gluten-free diet. Pediatric Research, 6, 521-527.

Bass, S., Delmas, P. D., Pearce, G., Hendrich, E., Tabensky, A., \& Seeman, E. (1999). The differing tempo of growth in bone size, mass, and density in girls is regionspecific. Journal of Clinical Investigation, 104(6), 795-804.

Bass, S. L. (2003). The structural adaptations of cortical bone to loading during different stages of maturation. Journal of Musculoskeletal and Neuronal Interactions, 3(4), $345-347$.

Bass, S. L., Saxon, L., Daly, R. M., Turner, C. H., Robling, A. G., Seeman, E., et al. (2002). The effect of mechanical loading on the size and shape of bone in pre-, peri-, and postpubertal girls: a study in tennis players. Journal of Bone and Mineral Research, 17(12), 2274-2280.

Bertram, J. E., \& Swartz, S. M. (1991). The "Law of bone transformation”. Biological Reviews of Cambridge Philosophical Society, 66, 245-273. 
Biknevicius, A. R., \& Ruff, C. B. (1992). Use of biplanar radiographs for estimating cross-sectional geometric properties of mandibles. The Anatomical Record, 232(1), 157-163.

Binneman, J. (2004). Archaeological research along the southern-eastern Cape Coast part 1: Open-air shell middens. Southern African Field Archaeology, 13/14, 49-77.

Buikstra, J. E., \& Ubelaker, D. H. (1994). Standards for Data Collection from Human Skeletal Remains. Arkansas Archeological Survey Research Series No.44. Fayetteville: Arkansas Archeological Survey.

Carter, D. R., \& Beaupré, G. S. (2001). Skeletal function and form. Cambridge: Cambridge University Press.

Churchill, S. E., Formicola, V., Holliday, T. W., Holt, B. M., \& Schumann, B. (2000). The Upper Paleolithic population of Europe in an evolutionary perspective. In W. Roebroeks, M. Mussi, J. Svoboda, \& K. Fennema (Eds.), Hunters of the Golden Age: the Mid Upper Paleolithic of Eurasia (30,000-20,000 bp) (pp. 31-57). Leiden: Leiden University Press.

Clayton, F., Sealy, J., \& Pfeiffer, S. (2006). Weaning age among foragers at Matjes river rock shelter, South Africa, from stable nitrogen and carbon isotope analyses. American Journal of Physical Anthropology, 129(2), 311-317.

Cleveland, W. S., \& Devlin, S. J. (1988). Locally weighted regression: an approach to regression analysis by local fitting. Journal of the American Statistical 
Association, 83(403), 596-610.

Cowgill, L. W. (2006). Postcranial growth and development of immature skeletons from Point Hope, Alaska. American Journal of Physical Anthropology, 129(S42), 78.

Cowgill, L. W. (2007). Humeral torsion revisited: A functional and ontogenetic model for populational variation. American Journal of Physical Anthropology, 134(4), 472480.

Cowgill, L. W. (2008). The ontogeny of recent and Late Pleistocene human postcranial robusticity. Ph.D. Thesis, Washington University in St. Louis.

Cowgill, L. W., \& Hager, L. D. (2005). Variation in the development of post-cranial robusticity: an example from Çatalhöyük, Turkey. American Journal of Physical Anthropology, 126(S40), 28.

Cowgill, L. W., \& Hager, L. D. (2007). Variation in the development of postcranial robusticity: an example from Çatalhöyük, Turkey. International Journal of Osteoarchaeology, 17(3), 235-252.

Cowgill, L. W. (In Press). The ontogeny of Holocene and Late Pleistocene human postcranial strength. American Journal of Physical Anthropology.

Deacon, H. J. (1976). Where Hunters Gathered: A Study of Holocene Stone Age People in the Eastern Cape. Cape Town: South African Archaeological Society.

Deacon, H. J., \& Deacon, J. (1999). Human Beginnings in South Africa: Uncovering the Secrets of the Stone Age: Uncovering the Secrets of the Stone Age. Cape Town: 
David Phillip Publishers.

Dewar, G., \& Pfeiffer, S. (2004). Postural Behaviour of Later Stone Age People in South Africa. The South African Archaeological Bulletin, 59(180), 52-58.

Draper, P. (1976). Social and economic constraints on child life among the !Kung. In Kalahari Hunter-Gatherers: Studies of the !Kung San and Their Neighbors (pp. 199-217). Cambridge: Harvard University Press.

Draper, P., \& Howell, N. (2005). The growth and kinship resources of Ju/'hoansi children. In M. E. Lamb \& B. S. Hewlett (Eds.), Hunter-Gatherer Childhoods: Evolutionary, Developmental \& Cultural Perspectives (pp. 262-281). New Brunswick, New Jersey: Aldine Transaction.

Dupras, T. L. (2003). The Moatfield infant and juvenile skeletal remains. In R. F. Williamson \& S. Pfeiffer (Eds.), Bones of the Ancestors: The Archaeology and Osteobiography of the Moatfield Ossuary (pp. 295-308). Gatineau, Quebec: Canadian Museum of Civilization.

Ericksen, M. F. (1976). Cortical bone loss with age in three native American populations. American Journal of Physical Anthropology, 45(3), 443-452.

Frost, H. M. (2003). Bone's mechanostat: A 2003 update. The Anatomical Record Part A: Discoveries in Molecular, Cellular, and Evolutionary Biology, 275A(2), 10811101.

Frost, H. M. (1999). Changing views about 'osteoporoses' (a 1998 overview). 
Osteoporosis International, 10, 345-352.

Frost, H. M. (1987). The mechanostat: a proposed pathogenic mechanism of osteoporoses and the bone mass effects of mechanical and nonmechanical agents. Bone and Mineral, 2(2), 73-85.

Garn, S. M. (1970). The Earlier Gain and the Later Loss of Cortical Bone in Nutritional Perspective. Springfield: Charles C. Thomas.

Garn, S. M., Guzman, M. A., \& Wagner, B. (1969). Subperiosteal gain and endosteal loss in protein-calorie malnutrition. American Journal of Physical Anthropology, $30(1), 153-155$.

Haapasalo, H., Kannus, P., Sievänen, H., Pasanen, M., Uusi-Rasi, K., Heinonen, A., et al. (1998). Effect of long-term unilateral activity on bone mineral density of female junior tennis players. Journal of Bone and Mineral Research, 13(2), 310-319.

Harrington, L., \& Pfeiffer, S. (2008). Juvenile mortality in southern African archaeological contexts. South African Archaeological Bulletin, 63(188), 95-101.

Himes, J., Martorell, R., Habicht, J., Yarbrough, C., Malina, R., \& Klein, R. (1976). Sexual dimorphism in bone growth as a function of body size in moderatelly malnourished Guatemalan preschool age children. American Journal of Physical Anthropology, 45, 331-336.

Holliday, T. W. (2002). Body size and postcranial robusticity of European Upper Paleolithic hominins. Journal of Human Evolution, 43, 513-528. 
Holt, B. M. (2003). Mobility in Upper Paleolithic and Mesolithic Europe: Evidence from the lower limb. American Journal of Physical Anthropology, 122(3), 200-215.

Holt, B. M. (1999). Biomechanical evidence of decreased mobility in Upper Paleolithic and Mesolithic Europe (Ph.D.). University Missouri-Columbia.

Hoppa, R. D., \& FitzGerald, C. M. (1999). From head to toe: integrating studies from bones and teeth in biological anthropology. In R. D. Hoppa \& C. M. FitzGerald (Eds.), Human Growth in the Past: Studies from Bones and Teeth (pp. 1-31). Cambridge: Cambridge University Press.

Howell, N. (1979). Demography of the Dobe !Kung (1st ed.). New York: Academic Press. Howell, N. (2000). Demography of the Dobe !Kung. Evolutionary Foundations of Human Behavior (2nd ed.). Aldine Transaction.

Huiskes, R. (1982). On the modelling of long bones in structural analyses. Journal of Biomechanics, 15(1), 65-69.

Hummert, J. R. (1983). Cortical bone growth and dietary stress among subadults from Nubia's Batn El Hajar. American Journal of Physical Anthropology, 62, 167-176.

Konigsberg, L. W., Hens, S. M., Jantz, L. M., \& Jungers, W. L. (1998). Stature estimation and calibration: Bayesian and maximum likelihood perspectives in physical anthropology. American Journal of Physical Anthropology, 107(S27), 65-92.

Konner, M. (2005). Hunter-gather infancy and childhood: the !Kung and others. In B. S. Hewlett \& M. E. Lamb (Eds.), Hunter-Gatherer Childhoods: Evolutionary, 
Developmental \& Cultural Perspectives (pp. 19-64). Aldine Transaction.

Konner, M. (1976). Maternal care, infant behavior and development among the !Kung. In R. B. Lee \& I. Devore (Eds.), Kalahari Hunter-Gatherers: Studies of the !Kung San and Their Neighbors (pp. 218-245). Cambridge, Massachusetts: Harvard University Press.

Kontulainen, S., Sievänen, H., Kannus, P., Pasanen, M., \& Vuori, I. (2002). Effect of long-term impact-loading on mass, size, and estimated strength of humerus and radius of female racquet-sports players: a peripheral quantitative computed tomography study between young and old starters and controls. Journal of Bone and Mineral Research, 17(12), 2281-2289.

Larsen, C. S. (1997). Bioarchaeology: Interpreting Behavior from the Human Skeleton. Cambridge: Cambridge University Press.

Lazenby, R. A. (1990). Continuing periosteal apposition II: The significance of peak bone mass, strain equilibrium, and age-related activity differentials for mechanical compensation in human tubular bones. American Journal of Physical Anthropology, 82(4), 473-484.

Lee, R. B. (1979). The !Kung San: Men, Women and Work in a Foraging Society. Cambridge: Cambridge University Press.

Lieberman, D. E., Polk, J. D., \& Demes, B. (2004). Predicting long bone loading from cross-sectional geometry. American Journal of Physical Anthropology, 123(2), 
$156-171$.

Lieberman, D. E., Pearson, O. M., Polk, J. D., Demes, B., \& Crompton, A. W. (2003). Optimization of bone growth and remodeling in response to loading in tapered mammalian limbs. Journal of Experimental Biology, 206(18), 3125-3138.

Lovejoy, O. C. (1975). Biomechanical perspectives on the lower limb of early hominids. In R. H. Tuttle (Ed.), Primate Functional Morphology and Evolution (pp. 291326). The Hague: Mouton.

MacKelvie, K., Khan, K., McKay, H., \& Sanborn, C. (2002). Is there a critical period for bone response to weight-bearing exercise in children and adolescents? A systematic review. British Journal of Sports Medicine, 36(4), 250-257.

Malina, R. (2002). Exercise and growth: Physical activity as a factor in growth and maturation. In N. Cameron (Ed.), Human Growth and Development (pp. 321-348). San Diego: Academic Press.

Maresh, M. M. (1943). Growth of major long bones in healthy children: a preliminary report on successive roentgenograms of the extremities from early infancy to twelve years of age. American Journal of Diseases of Children, 66(3), 227-257.

Maresh, M. M. (1955). Linear growth of long bones of extremities from infancy through adolescence; continuing studies. American Journal of Diseases of Children, 89(6), $725-742$.

Maresh, M. M. (1970). Measurements from Roentgenograms. In M. M. Maresh \& R. W. 
Mccammon (Eds.), Human Growth and Development (pp. 157-200). Springfield, Illinois: Charles C. Thomas.

Matzke, L. (2000). Dental development and maturation of juvenile dentitions from the Later Stone Age, South Africa. M.Sc. Thesis, University of Toronto.

Mays, S. (1995). The relationship between Harris lines and other aspects of skeletal development in adults and juveniles. Journal of Archaeological Science, 22, 511520.

Mays, S. (1999). Linear and appositional long bone growth in earlier human p. In R. D. Hoppa \& C. M. FitzGerald (Eds.), Human Growth in the Past: Studies from Bones and Teeth (pp. 290-312). Cambridge: Cambridge University Press.

Meadows, M. E., \& Sugden, J. M. (1993). The late quaternary palaeoecology of a floristic kingdom: the southwestern Cape South Africa. Palaeogeography, Palaeoclimatology, Palaeoecology, 101(3-4), 271-281.

Meehan, B. (1982). Shell Bed to Shell Midden. Canberra: Australian Institute of Aboriginal Studies.

Milgrom, C. (1989). The area moment of inertia of the tibia: A risk factor for stress fractures. Journal of Biomechanics, 22(11-12), 1243-1248.

Mitchell, P. (2002). The Archaeology of Southern Africa. Cambridge: Cambridge University Press.

Moorrees, C. F. A., Fanning, E. A., \& Hunt, E. E. (1963a). Formation and resorption of 
three deciduous teeth in children. American Journal of Physical Anthropology, 21(2), 205-213.

Moorrees, C. F. A., Fanning, E. A., \& Hunt, E. E. (1963b). Age variation of formation stages for ten permanent teeth. Journal of Dental Research, 42(6), 1490-1502.

Noli, D., \& Avery, G. (1988). Protein poisoning and coastal subsistence. Journal of Archaeological Science, 15(4), 395-401.

Ohman, J. C. (1993). Computer software for estimating cross-sectional geometric properties of long bones with concentric and eccentric elliptical models. Journal of Human Evolution, 25(3), 217-227.

Parkington, J. (1998). Resolving the past: Gender in the archaeological record of the Western Cape. In S. Kent (Ed.), Gender in African Prehistory (pp. 25-38). Walnut Creek: Altamira Press.

Parkington, J. (1972). Seasonal mobility in the Late Stone Age. African Studies, 31, 151158.

Parkington, J. (2001). Mobility, seasonality and southern African hunter-gatherers. South African Archaeological Bulletin, 56, 1-7.

Pearson, O. M., \& Lieberman, D. E. (2004). The aging of Wolff's "law": Ontogeny and responses to mechanical loading in cortical bone. American Journal of Physical Anthropology, 125(S39), 63-99.

Pfeiffer, S., \& Sealy, J. (2006). Body size among Holocene foragers of the Cape Ecozone, 
southern Africa. American Journal of Physical Anthropology, 129(1), 1-11.

Pfeiffer, S. (2007). The health of foragers: People of the Later Stone Age, southern Africa. In M. N. Cohen \& Gillian (Eds.), Ancient Health: Skeletal Indicators of Agricultural and Economic Intensification, Bioarchaeological Interpretations of the Human Past: Local, Regional, and Global Perspectives (pp. 223-236). University Press of Florida.

Pfeiffer, S., \& Stock, J. T. (2002). Upper limb morphology and the division of labor among southern African Holocene foragers. American Journal of Physical Anthropology, S34, 124.

Rasband, W. S. (n.d.). ImageJ. Bathesda, Maryland: U.S. National Institutes of Health. Retrieved from http://rsb.info.nih.gov/ij/

Rauch, F., \& Schöenau, E. (2001). Changes in Bone Density During Childhood and Adolescence: An Approach Based on Bone's Biological Organization. Journal of Bone and Mineral Research, 16(4), 597-604.

Rubin, C. T., \& Lanyon, L. E. (1984). Regulation of bone formation by applied dynamic loads. Journal of Bone and Joint Surgery, 66, 397-402.

Ruff, C., Niskanen, M., Junno, J. A., \& Jamison, P. (2005). Body mass prediction from stature and bi-iliac breadth in two high latitude populations, with application to earlier higher latitude humans. Journal of Human Evolution, 48(4), 381-392.

Ruff, C. B., \& Hayes, W. C. (1983). Cross-sectional geometry of Pecos Pueblo femora 
and tibiae-a biomechanical investigation: II. Sex, age, side differences. American journal of physical anthropology, 60(3), 383-400.

Ruff, C. B., Trinkaus, E., Walker, A., \& Larsen, C. S. (1993). Postcranial robusticity in Homo I: Temporal trends and mechanical interpretation. American Journal of Physical Anthropology, 91(1), 21-53.

Ruff, C. (2007). Body size prediction from juvenile skeletal remains. American Journal of Physical Anthropology, 133(1), 698-716.

Ruff, C., Holt, B., \& Trinkaus, E. (2006). Who's afraid of the big bad Wolff?: Wolff's law and bone functional adaptation. American Journal of Physical Anthropology, 129(4), 484-498.

Ruff, C. B. (2008). Biomechanical analyses of archaeological human skeletons. In M. A. Katzenberg \& S. R. Saunders (Eds.), Biological Anthropology of the Human Skeleton (2nd ed.). New York: Wiley-Liss.

Ruff, C. B., \& Hayes, W. C. (1983). Cross-sectional geometry of Pecos Pueblo femora and tibiae - A biomechanical investigation: I. Method and general patterns of variation. American Journal of Physical Anthropology, 60(3), 359-381.

Ruff, C. B. (1991). Aging and Osteoporosis in Native Americans from Pecos Pueblo. New York: Garland Publishing.

Ruff, C. B. (n.d.). MomentMacroJ. Retrieved from http://www.hopkinsmedicine.org/FAE/mmacro.htm/ 
Ruff, C. B., Walker, A., \& Trinkaus, E. (1994). Postcranial robusticity in Homo III: Ontogeny. American Journal of Physical Anthropology, 93(1), 35-54.

Runestad, J. A., Ruff, C. B., Nieh, J. C., Thorington, R. W., \& Teaford, M. F. (1993). Radiographic estimation of long bone cross-sectional geometric properties. American Journal of Physical Anthropology, 90(2), 207-213.

Schapera, I. (1930). The Khoisan Peoples of South Africa. London: Routledge and Paul Kegan.

Scheuer, L., \& Black, S. (2000). Developmental Juvenile Osteology. San Diego: Academic Press.

Scheuer, L., \& Black, S. (2004). The Dentition, with contributions from Helen Liversidge. In The Juvenile Skeleton (pp. 149-180). San Diego: Academic Press.

Sealy, J. C. (1995). Analysing the past: Modern analytical techniques, notably involving the measurement of isotope ratios, help clarify early mysteries regarding diet and trading patterns. South African Journal of Science, 91, 11-12.

Sealy, J. C. (1997). Stable carbon and nitrogen isotope ratios and coastal diets in the Later Stone Age of South Africa: A comparison and critical analysis of two data sets. Ancient Biomolecules, 1, 131-147.

Sealy, J. C. (2006). Diet, mobility, and settlement pattern in Holocene South Africa. Current Anthropology, 47(4), 569-595.

Sealy, J. C., \& van der Merwe, N. J. (1988). Social, spatial and chronological patterning 
in marine food use as determined by delta $13 \mathrm{C}$ measurements of Holocene human skeletons from the south-western Cape, South Africa. World Archaeology, 20(1), $87-102$.

Sealy, J. C., \& Pfeiffer, S. (2000). Diet, body size, and landscape use among Holocene people in the Southern Cape, South Africa. Current Anthropology, 41(4), 641655.

Shackelford, L. L. (2005). Regional variation in the postcranial robusticity of Late Upper Paleolithic humans. Ph.D. Thesis, Washington University.

Shackelford, L. L. (2007). Regional variation in the postcranial robusticity of late upper paleolithic humans. American Journal of Physical Anthropology, 133(1), 655668.

Shostak, M. (1981). Nisa: The Life and Words of a!Kung Woman (1st ed.). Harvard University Press.

Silberbauer, G. B. (1981). Hunter and Habitat in the Central Kalahari Desert. Cambridge: Cambridge University Press.

Singer, R., \& Wymer, J. (1982). The Middle Stone Age at Klasies River Mouth in South Africa. Chicago: University of Chicago Press.

Smith, B. H. (1991). Standards of human tooth formation and dental age assessment. In M. A. Kelley \& C. S. Larsen (Eds.), Advances in Dental Anthropology (pp. 143168). New York: Wiley-Liss. 
Steinberg, M. E., \& Trueta, J. (1981). Effects of activity on bone growth and development in the rat. Clinical Orthopaedics and Related Research, (156), 52-60.

Stock, J., \& Pfeiffer, S. (2001). Linking structural variability in long bone diaphyses to habitual behaviors: Foragers from the southern African Later Stone Age and the Andaman Islands. American Journal of Physical Anthropology, 115(4), 337-348.

Stock, J. T. (2002a). Climatic and behavioural influences on postcranial robusticity among Holocene foragers. University of Toronto.

Stock, J. T. (2002b). A test of two methods of radiographically deriving long bone crosssectional properties compared to direct sectioning of the diaphysis. International Journal of Osteoarchaeology, 12(5), 335-342.

Stock, J. T., \& Pfeiffer, S. K. (2004). Long bone robusticity and subsistence behaviour among Later Stone Age foragers of the forest and fynbos biomes of South Africa. Journal of Archaeological Science, 31(7), 999-1013.

Thompson, J. L., \& Nelson, A. J. (2000). The place of Neandertals in the evolution of hominid patterns of growth and development. Journal of Human Evolution, 38(4), $475-495$.

Trinkaus, E., \& Ruff, C. B. (1989). Diaphyseal cross-sectional morphology and biomechanics of the Fond-de-Forêt 1 femur and the Spy 2 femur and tibia. Bulletin de la Societé Royale Belge d'Anthropologie et de Prehistoire, 100, 33-42.

Trinkaus, E., \& Churchill, S. E. (1999). Diaphyseal Cross-sectional Geometry of Near 
Eastern Middle Palaeolithic Humans: The Humerus. Journal of Archaeological Science, 26(2), 173-184.

Trinkaus, E., \& Ruff, C. B. (1999). Diaphyseal Cross-sectional Geometry of Near Eastern Middle Palaeolithic Humans: The Tibia. Journal of Archaeological Science, 26(10), 1289-1300.

Truswell, A. S., \& Hanson, J. D. L. (1976). Medical research among the !Kung. In R. B. Lee \& I. Devore (Eds.), Kalahari Hunter-Gatherers (pp. 166-194). Cambridge, Massachusetts: Harvard University Press.

Ubelaker, D. H. (1989). Human Skeletal Remains: Excavation, Analysis, Interpretation (2nd ed.). Washington: Taraxacum.

Van Gerven, D. P., Hummert, J. R., \& Burr, D. B. (1985). Cortical bone maintenance and geometry of the tibia in prehistoric children from Nubia's Batn el Hajar. American Journal of Physical Anthropology, 66(3), 275-280.

Wadley, L. (1998). The invisible meat providers: Women in the Stone Age of South Africa. In S. Kent (Ed.), Gender in African Prehistory (pp. 69-82). Walnut Creek: Altamira Press.

Wainwright, S. A. (1982). Mechanical design in organisms. Princeton: Princeton University Press.

Wolff, J. (1892). Das Geetz der Transformation der Knochen. Berlin: A. Hirchwild. Wolff, J. (1986). The Law of Bone Remodelling. Berlin: Springer-Verlag. 
Wood, J. W., Milner, G. R., Harpending, H. C., \& Weiss, K. M. (1992). The osteological paradox: Problems of inferring prehistoric health from skeletal samples. Current Anthropology, 33(4), 343-370. 


\section{Appendix A. Later Stone Age juveniles included in the study sample}

\begin{tabular}{|c|c|c|c|c|c|c|}
\hline Catalogue & Curation 1 & Site & $\begin{array}{c}\text { Age Estimate } \\
\text { (years) }\end{array}$ & Age Method $^{2}$ & $\begin{array}{c}\text { Mass Estimate } \\
(\mathrm{kg})\end{array}$ & Mass Method 2,3 \\
\hline WSK1 Infant* & NMB & Matjes River & -0.75 & Predicted from HD & 4.29 & Predicted FDM from TPM \\
\hline UCT $188 / 217 \mathrm{~N}$ & UCT & Oakhurst & -0.52 & Predicted from HD & 3.70 & FDM \\
\hline ALB 337 & ALB & Klasies River Mouth & -0.37 & Predicted from HD & 4.76 & FDM \\
\hline $\mathrm{S} 4 / 26 / 3^{\prime} 6^{\prime \prime *}$ & NMB & Matjes River & -0.15 & Predicted from HD & 4.28 & Predicted FDM from CM \\
\hline NMB 1444 & NMB & Matjes River & 0.16 & DD & 3.87 & Predicted FDM from TPM \\
\hline ALB 297 & ALB & Klasies River Mouth 5 & 0.22 & DD & 4.22 & Predicted FDM from CM \\
\hline $5 \mathrm{~V}^{*}$ & NMB & Matjes River & 0.25 & DD & 5.46 & FDM \\
\hline NMB 1643 & NMB & Robberg Cave & 0.25 & DD & 3.29 & Predicted FDM from CM \\
\hline NMB 1644 & NMB & Robberg Cave & 0.25 & DD & 3.00 & FDM \\
\hline UCT $217 \mathrm{M} / 187$ & UCT & Oakhurst & 0.25 & DD & 3.74 & FDM \\
\hline ALB 298 & ALB & Klasies River Mouth 5 & 0.27 & DD & 4.45 & Predicted FDM from CM \\
\hline S 592* & NMB & Matjes River & 0.38 & DD & 5.90 & FDM \\
\hline ALB 299 & ALB & Klasies River Mouth 5 & 0.41 & DD & 5.76 & FDM \\
\hline OKT $58^{*}$ & NMB & Matjes River & 0.52 & DD & 5.02 & FDM \\
\hline UCT $217 \mathrm{~L} / 189$ & UCT & Oakhurst & 0.54 & DD & 5.54 & FDM \\
\hline ALB 322 & ALB & Klasies River Mouth & 0.61 & DD & 5.24 & FDM \\
\hline SAM AP 4829 & SAM & Tucker's Cave & 0.74 & DD & 8.17 & FDM \\
\hline ALB 135 & ALB & Spitzkop & 1.11 & DD & 7.54 & FDM \\
\hline UCT $217 \mathrm{~K} / 190$ & UCT & Oakhurst & 1.22 & DD & 6.89 & FDM \\
\hline ALB 311 & ALB & Welgeluk & 1.30 & DD & 8.41 & FDM \\
\hline ALB 319B & ALB & Joubertina & 1.52 & DD & 10.06 & FDM \\
\hline ALB 265 & ALB & New Gloucester & 1.58 & DD & 6.48 & FDM \\
\hline ALB 116B & ALB & Wilton Large Rock Shelter & 1.81 & Predicted from HD & 7.17 & FDM \\
\hline SAM AP $6054 C$ & SAM & Modder River Mouth & 1.82 & DD & 7.54 & FDM \\
\hline UCT 346 & $\mathrm{UCT}$ & Nelson Bay Cave & 2.23 & DD & 10.90 & FDM \\
\hline UCT 196/207H & UCT & Oakhurst & 2.45 & DD & 10.64 & FDM \\
\hline ALB 195B & ALB & Melkhoutboom & 2.46 & DD & 8.70 & FDM \\
\hline ALB 138 & ALB & Spitzkop & 3.55 & DD & 10.76 & FDM \\
\hline ALB 137 & ALB & Spitzkop & 4.15 & DD & 11.84 & FDM \\
\hline S5 PROTO* & NMB & Matjes River & 4.35 & DD & 12.68 & FDM \\
\hline ALB 236 & ALB & Mitford Park & 4.80 & DD & 9.06 & FDM \\
\hline NMB 1641 & NMB & Robberg Cave & 5.15 & Predicted from HD & 14.04 & FDM \\
\hline UCT 437 & UCT & Kasteelberg & 5.25 & DD & 12.00 & FDM \\
\hline ALB 122 & ALB & Wilton Large Rock Shelter & 5.26 & DD & 12.93 & FDM \\
\hline
\end{tabular}




\begin{tabular}{|c|c|c|c|c|c|c|}
\hline Catalogue & Curation 1 & Site & $\begin{array}{c}\text { Age Estimate } \\
\text { (years) }\end{array}$ & Age Method ${ }^{2}$ & $\begin{array}{c}\text { Mass Estimate } \\
(\mathrm{kg})\end{array}$ & Mass Method ${ }^{2,3}$ \\
\hline NMB 1642 & NMB & Robberg Cave & 5.64 & Predicted from HD & 17.28 & FDM \\
\hline SAM AP 6054B & SAM & Modder River Mouth & 5.69 & DD & 13.79 & Predicted FDM from TPM \\
\hline SAM AP 4207 & SAM & Cape Town Riebeeck St. & 5.91 & DD & 14.83 & Predicted FDM from TPM \\
\hline SAM AP 6052 & SAM & Byneskranskop & 5.95 & DD & 14.73 & FDM \\
\hline Skeleton \#4* & NMB & Matjes River & 6.25 & DD & 18.15 & FDM \\
\hline ALB 132 & ALB & Spitzkop & 6.45 & DD & 16.03 & FDM \\
\hline UCT 388 & UCT & Faraoskop & 6.50 & DD & 19.50 & FHE \\
\hline ALB 205 & ALB & Middlekop & 6.61 & DD & 18.93 & Predicted FHE from TPE \\
\hline ALB 133 & ALB & Spitzkop & 6.70 & DD & 17.95 & FHE \\
\hline SAM AP 6053 & SAM & Byneskranskop 1 & 7.53 & DD & 26.71 & FHE \\
\hline MRSK* & NMB & Matjes River & 7.68 & Predicted from FD & 24.48 & Predicted FHE from CM \\
\hline Wilton Up Strat A* & NMB & Matjes River & 7.78 & Predicted from UD & 22.79 & Predicted FHE from UD \\
\hline ALB 350 & ALB & Oyster Bay & 8.3 & DD & 22.33 & Predicted FHE from TPM \\
\hline UCT 191/217E & UCT & Oakhurst & 8.38 & Predicted from HD & 23.49 & FHE \\
\hline Wilton Up Strat B* & NMB & Matjes River & 9.10 & Predicted from HD & 20.09 & Predicted FHE from TPM \\
\hline ALB 238 & ALB & Widcome & 10.07 & Predicted from HD & 25.19 & FHE \\
\hline ALB 151B & ALB & near Kabeljaauws & 10.85 & DD & 26.44 & FHE \\
\hline ALB 51 & ALB & Plettenberg Bay & 10.93 & DD & 18.65 & FHE \\
\hline ALB 319A & ALB & Joubertina & 11.06 & Predicted from CM & 30.27 & FHE \\
\hline ALB 351 & ALB & Papiesfontein & 11.20 & DD & 34.28 & FHE \\
\hline ALB 120 & ALB & Wilton Cave & 11.40 & DD & 34.58 & FHE \\
\hline SAM AP 6054A & SAM & Modder River Mouth & 12.20 & DD & 18.49 & FHE \\
\hline ALB128E & ALB & Spitzkop & 12.30 & Predicted from TD & 35.34 & Predicted FHE from UD \\
\hline SAM AP 6085 & SAM & Milnerton Beach & 12.39 & Predicted from TD & 39.08 & FHE \\
\hline UCT 110 & UCT & Cape Town Sea Point & 12.44 & Predicted from HD & 36.05 & FHE \\
\hline ALB 152/161B & ALB & Kabeljaauws & 13.10 & DD & 22.96 & Predicted FHE from TPE \\
\hline ALB 199 & ALB & Middlekop & 13.10 & DD & 39.96 & FHE \\
\hline SAM AP 6348A & SAM & Melkbos & 14.32 & Predicted from HD & 31.65 & FHE \\
\hline $\mathrm{SS} 2 *$ & NMB & Matjes River & 14.71 & Predicted from UM & 32.20 & FHE \\
\hline Skeleton $\# 1^{*}$ & NMB & Matjes River & 14.84 & Predicted from UM & 37.95 & FHE \\
\hline NMB 1640 & NMB & Robberg Cave & 14.92 & Predicted from UM & 38.01 & FHE \\
\hline ALB 139 & ALB & Spitzkop & 15.00 & Predicted from UM & 35.79 & FHE \\
\hline ALB 195A & ALB & Melkhoutboom & 15.07 & Predicted from UM & 35.57 & Predicted FHE from UM \\
\hline NMB 1273 & NMB & Matjes River & 15.38 & Predicted from UM & 37.78 & Predicted FHE from UM \\
\hline SAM AP 4210 & SAM & Drury's Cave & 15.41 & Predicted from UM & 34.27 & FHE \\
\hline NMB 1271 & NMB & Matjes River & 15.46 & Predicted from UM & 41.75 & FHE \\
\hline ALB 222 & ALB & Seal Point Cape St. Francis & 15.49 & Predicted from UM & 39.26 & FHE \\
\hline SAM AP 4212A & SAM & Drury's Cave & 15.85 & Predicted from UM & 46.43 & FHE \\
\hline
\end{tabular}




\begin{tabular}{|l|c|l|c|l|c|l|}
\hline Catalogue & Curation $\mathbf{1}$ & Site & $\begin{array}{c}\text { Age Estimate } \\
\text { (years) }\end{array}$ & Age Method $^{\mathbf{2}}$ & $\begin{array}{c}\text { Mass Estimate } \\
\text { (kg) }\end{array}$ & Mass Method $^{\mathbf{2}, \mathbf{3}}$ \\
\hline SAM AP 6083A & SAM & Milnerton Beach & 15.85 & Predicted from UM & 34.75 & Predicted FHE from TPE \\
\hline ALB 198 & ALB & Middlekop Kloof & 15.90 & DD & 48.25 & FHE \\
\hline ALB 296 & ALB & Klasies River Cave & 15.90 & DD & 45.55 & FHE \\
\hline SAM AP 4304B & SAM & Noordhoek & 15.90 & DD & 48.25 & FHE \\
\hline ALB 354 & ALB & Paradysstrand & 15.90 & Predicted from UM & 34.75 & FHE \\
\hline UCT 162 & UCT & Ysterfontein & 16.27 & DD & 45.55 & FHE \\
\hline ALB 151A & ALB & near Kabeljaauws & 16.65 & DD & 45.33 & Predicted FHE from UM \\
\hline ALB 200 & ALB & Middlekop Kloof & 16.65 & DD & 51.67 & FHE \\
\hline UCT 200 & UCT & Oakhurst & 17.09 & Predicted from UM & 51.81 & FHE \\
\hline ALB 119 & ALB & Wilton Large Rock Shelter & 18.27 & Predicted from CM & 45.46 & FHE \\
\hline
\end{tabular}

*Burial not accessioned by curatorial institution

${ }^{1} \mathrm{ALB}=$ Albany Museum, NMB = National Museum Bloemfontein, UCT = University of Cape Town, SAM = Iziko South African Museum

${ }^{2} \mathrm{DD}=$ Dental development; Predicted from length of $\mathrm{CM}=$ Clavicle maximum, $\mathrm{FD}=$ Femur diaphysis, $\mathrm{FM}=$ Femur maximum, $\mathrm{HD}=\mathrm{Humerus}$ diaphysis, $\mathrm{HM}=$ Humerus maximum, $\mathrm{TD}=$ Tibia diaphysis, $\mathrm{TM}=$ Tibia maximum, $\mathrm{UD}=$ Ulna diaphysis, $\mathrm{UM}=$ Ulna maximum

${ }^{3}$ Predicted from maximal breadth of FDM $=$ Femur distal metaphysis, FHE $=$ Femur head epiphysis, TPE $=$ Tibia Proximal Epiphysis, TPM $=$ Tibia proximal metaphysis 


\section{Appendix B. Dental age prediction scatter plots and regression equations}
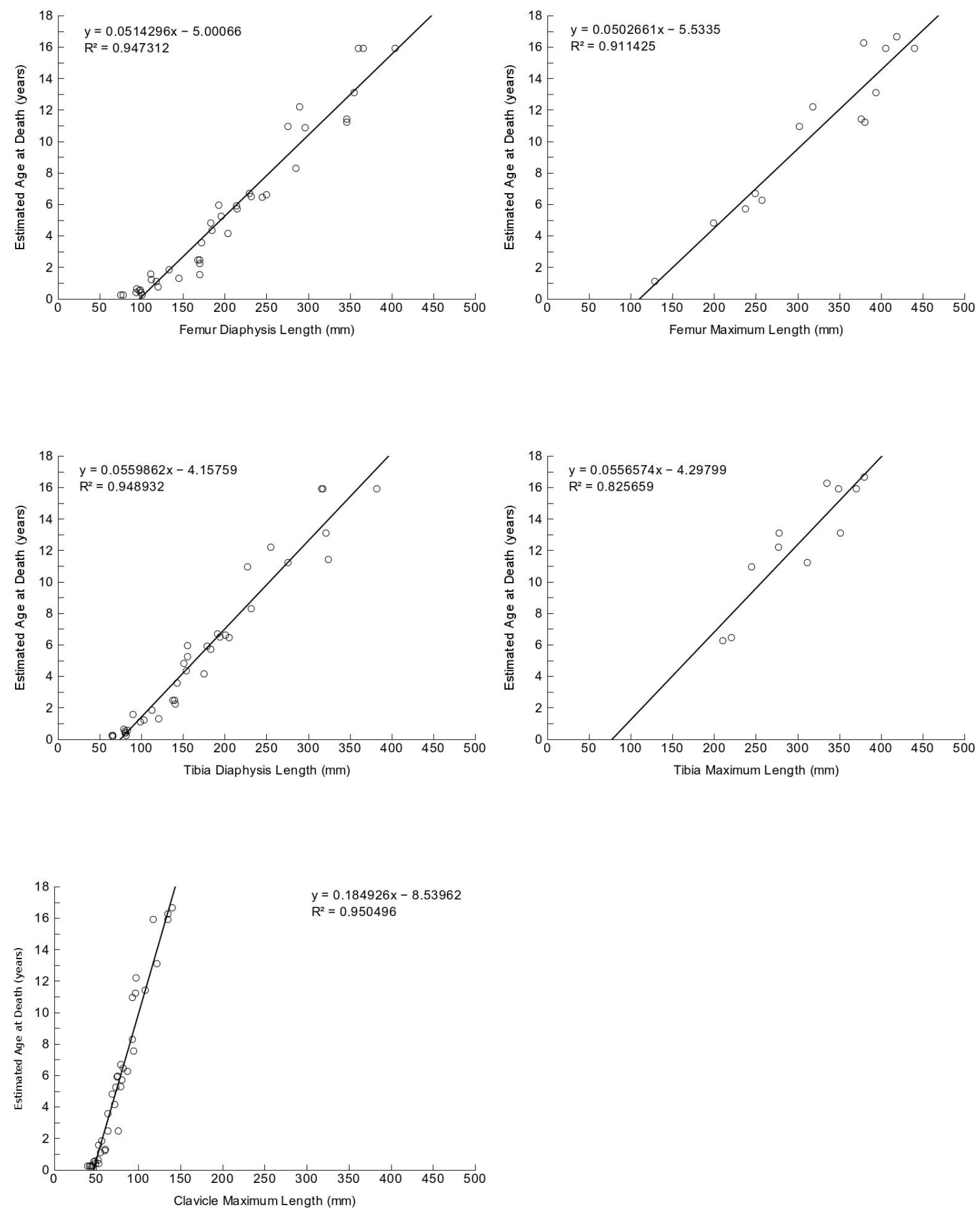

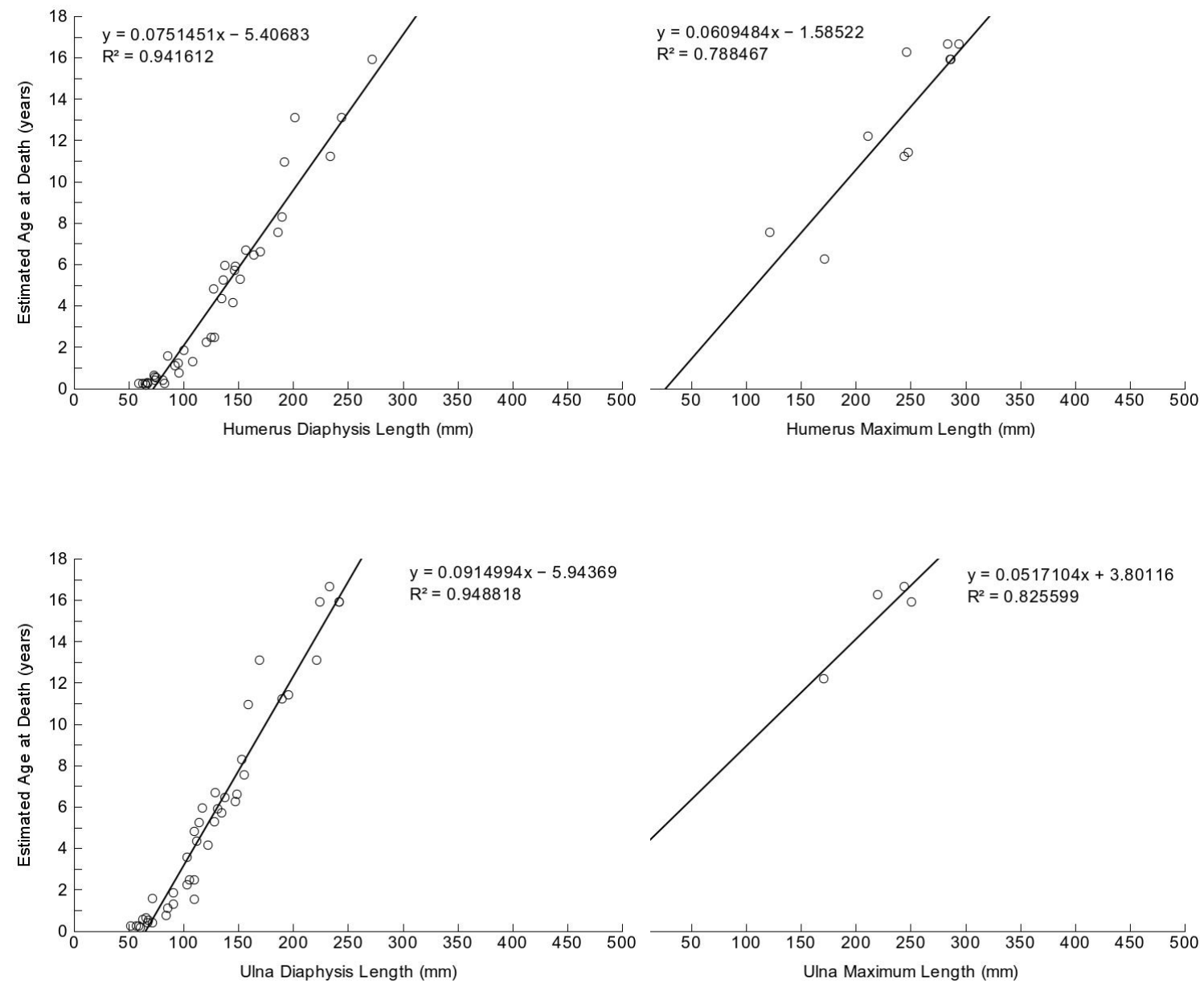


\section{Appendix C. Femoral dimensions in Later Stone Age and Denver Growth Study} juveniles

\begin{tabular}{|c|c|c|c|c|c|c|c|c|c|c|}
\hline \multirow{2}{*}{\begin{tabular}{c}
\multirow{2}{*}{$\begin{array}{c}\text { Age Cohort } \\
\text { (years) }\end{array}$} \\
\cline { 2 - 12 }
\end{tabular}} & \multicolumn{9}{|c|}{ LSA } \\
\cline { 2 - 11 } & $\mathbf{n}$ & mean & SD & Min & Max & n & mean & SD & Min & Max \\
\hline $\mathbf{0 . 1 7}$ & 2 & 20.67 & 1.15 & 19.86 & 21.49 & & & & & \\
\hline $\mathbf{0 . 3 3}$ & 5 & 23.73 & 4.14 & 18.04 & 27.20 & 15 & 27.6 & & 24.5 & 29.6 \\
\hline $\mathbf{0 . 5 0}$ & 4 & 26.98 & 3.23 & 24.67 & 31.73 & 20 & 34.3 & 2.6 & 27.9 & 38.4 \\
\hline $\mathbf{1}$ & 3 & 29.86 & 2.58 & 27.40 & 32.55 & 20 & 42.5 & 2.6 & 35.4 & 45.8 \\
\hline $\mathbf{2}$ & 6 & 33.29 & 5.32 & 26.95 & 40.29 & 20 & 47.1 & 2.4 & 41.9 & 50.3 \\
\hline $\mathbf{3}$ & & & & & & 19 & 49.4 & 2.6 & 43.4 & 52.8 \\
\hline $\mathbf{4}$ & 3 & 42.06 & 2.17 & 39.80 & 44.12 & 19 & 51.3 & 2.6 & 46.4 & 54.5 \\
\hline $\mathbf{5}$ & 3 & 41.29 & 5.61 & 35.33 & 46.46 & 18 & 53.7 & 2.8 & 48.1 & 57.7 \\
\hline $\mathbf{6}$ & 5 & 42.96 & 9.73 & 25.89 & 49.38 & 18 & 55.7 & 3.1 & 50.2 & 59.8 \\
\hline $\mathbf{7}$ & 3 & 43.55 & 0.79 & 42.97 & 44.45 & 18 & 57.5 & 3.0 & 52.1 & 62.2 \\
\hline $\mathbf{8}$ & 2 & 52.03 & 1.20 & 51.21 & 52.85 & 20 & 59.9 & 2.9 & 53.4 & 63.8 \\
\hline $\mathbf{9}$ & & & & & & 20 & 61.9 & 2.9 & 55.5 & 67.0 \\
\hline $\mathbf{1 0}$ & & & & & & 20 & 63.8 & 2.9 & 57.3 & 68.6 \\
\hline $\mathbf{1 1}$ & 3 & 57.05 & 6.12 & 50.00 & 60.89 & 20 & 66.0 & 2.6 & 59.2 & 69.4 \\
\hline $\mathbf{1 2}$ & 1 & 62.64 & & & & 20 & 67.9 & 3.0 & 62.6 & 74.9 \\
\hline $\mathbf{1 3}$ & 2 & 56.82 & 5.23 & 53.12 & 60.52 & & & & & \\
\hline $\mathbf{1 4}$ & 1 & 59.99 & & & & & & & & \\
\hline $\mathbf{1 5}$ & & & & & & & & & & \\
\hline $\mathbf{1 6}$ & 2 & 61.79 & 1.73 & 60.57 & 63.01 & & & & & \\
\hline $\mathbf{1 7}$ & & & & & & & & & & \\
\hline $\mathbf{1 8}$ & & & & & & & & & \\
\hline
\end{tabular}




\begin{tabular}{|c|c|c|c|c|c|c|c|c|c|c|}
\hline \multirow{3}{*}{$\begin{array}{c}\text { Age Cohort } \\
\text { (years) }\end{array}$} & \multicolumn{10}{|c|}{ Femoral Head Epiphysis Breadth } \\
\hline & \multicolumn{5}{|c|}{ LSA } & \multicolumn{5}{|c|}{ Denver } \\
\hline & $\mathbf{n}$ & mean & SD & Min & Max & $\mathbf{n}$ & mean & SD & Min & Max \\
\hline \multicolumn{11}{|l|}{0.17} \\
\hline \multicolumn{11}{|l|}{0.33} \\
\hline \multicolumn{11}{|l|}{0.50} \\
\hline 1 & 2 & 12.05 & 0.21 & 11.90 & 12.20 & & & & & \\
\hline 2 & 3 & 16.23 & 0.59 & 15.63 & 16.81 & & & & & \\
\hline \multicolumn{11}{|l|}{3} \\
\hline \multicolumn{11}{|l|}{4} \\
\hline 5 & 2 & 18.32 & 0.88 & 17.70 & 18.95 & & & & & \\
\hline 6 & 5 & 22.36 & 1.96 & 20.18 & 24.55 & & & & & \\
\hline 7 & 2 & 22.57 & 1.46 & 21.54 & 23.60 & 18 & 27.9 & 2.7 & 23.8 & 33.6 \\
\hline 8 & 2 & 30.08 & 2.40 & 28.36 & 31.80 & 18 & 30.1 & 2.8 & 25.0 & 35.8 \\
\hline 9 & & & & & & 20 & 32.4 & 2.9 & 27.5 & 38.0 \\
\hline 10 & 1 & 30.87 & & & & 20 & 34.5 & 2.9 & 29.5 & 40.8 \\
\hline 11 & 5 & 32.56 & 3.31 & 27.31 & 35.31 & 20 & 36.2 & 2.9 & 31.4 & 42.4 \\
\hline 12 & 3 & 34.68 & 5.76 & 28.08 & 38.64 & 20 & 38.3 & 3.1 & 32.9 & 44.2 \\
\hline 13 & 1 & 39.22 & & & & 20 & 40.4 & 3.1 & 35.5 & 46.0 \\
\hline 14 & 1 & 34.27 & & & & 20 & 41.8 & 2.9 & 36.5 & 48.2 \\
\hline 15 & 7 & 36.31 & 1.03 & 34.46 & 37.68 & 20 & 43.6 & 2.5 & 39.1 & 49.4 \\
\hline 16 & 6 & 38.10 & 2.03 & 35.35 & 40.46 & 20 & 44.5 & 2.8 & 38.8 & 50.1 \\
\hline 17 & 2 & 41.53 & 0.04 & 41.51 & 41.56 & 20 & 45.0 & 2.8 & 38.7 & 49.9 \\
\hline 18 & 1 & 39.15 & & & & & & & & \\
\hline
\end{tabular}




\begin{tabular}{|c|c|c|c|c|c|c|c|c|c|c|}
\hline \multirow{3}{*}{$\begin{array}{l}\text { Age Cohort } \\
\text { (years) }\end{array}$} & \multicolumn{10}{|c|}{ Femoral Diaphysis Length } \\
\hline & \multicolumn{5}{|c|}{ LSA } & \multicolumn{5}{|c|}{ Denver } \\
\hline & $\mathrm{n}$ & mean & SD & Min & Max & n & mean & SD & Min & Max \\
\hline 0.17 & 2 & 80.8 & 6.0 & 76.5 & 85.0 & & & & & \\
\hline 0.33 & 4 & 86.8 & 11.8 & 75.0 & 99.5 & & & & & \\
\hline 0.50 & 4 & 102.5 & 11.8 & 94.5 & 120.0 & & & & & \\
\hline 1 & 3 & 125.0 & 17.6 & 112.0 & 145.0 & 20 & 132.4 & 5.0 & 126.0 & 144.0 \\
\hline 2 & 7 & 149.9 & 25.2 & 111.0 & 170.0 & 20 & 166.7 & 7.1 & 156.0 & 179.0 \\
\hline 3 & & & & & & 20 & 192.8 & 8.6 & 178.0 & 209.0 \\
\hline 4 & 3 & 188.5 & 16.0 & 172.0 & 204.0 & 20 & 216.0 & 10.8 & 196.0 & 233.0 \\
\hline 5 & 3 & 193.2 & 8.9 & 183.0 & 199.5 & 20 & 237.1 & 11.9 & 216.0 & 256.0 \\
\hline 6 & 6 & 217.9 & 16.8 & 193.0 & 245.0 & 20 & 258.4 & 15.2 & 261.0 & 284.0 \\
\hline 7 & 3 & 237.0 & 11.3 & 229.5 & 250.0 & 20 & 278.5 & 16.4 & 246.0 & 301.0 \\
\hline 8 & 2 & 265.8 & 27.2 & 246.5 & 285.0 & 20 & 296.6 & 17.4 & 263.0 & 321.0 \\
\hline 9 & & & & & & 20 & 315.8 & 18.2 & 279.0 & 346.0 \\
\hline 10 & 1 & 300.0 & & & & 20 & 332.6 & 20.7 & 294.0 & 375.0 \\
\hline 11 & 4 & 316.0 & 35.6 & 276.0 & 346.0 & 20 & 349.4 & 22.2 & 311.0 & 397.0 \\
\hline 12 & 3 & 321.8 & 27.9 & 290.0 & 342.0 & 20 & 366.9 & 19.9 & 333.0 & 407.0 \\
\hline 13 & 1 & 358.5 & & & & & & & & \\
\hline 14 & 1 & 381.5 & & & & & & & & \\
\hline \multicolumn{11}{|l|}{15} \\
\hline 16 & 3 & 376.7 & 23.9 & 360.0 & 404.0 & & & & & \\
\hline \multicolumn{11}{|l|}{17} \\
\hline 18 & & & & & & & & & & \\
\hline
\end{tabular}




\begin{tabular}{|c|c|c|c|c|c|c|c|c|c|c|}
\hline \multirow{3}{*}{$\begin{array}{c}\text { Age Cohort } \\
\text { (years) }\end{array}$} & \multicolumn{10}{|c|}{ Femoral Maximum Length } \\
\hline & \multicolumn{5}{|c|}{ LSA } & \multicolumn{5}{|c|}{ Denver } \\
\hline & $\mathbf{n}$ & mean & SD & Min & $\operatorname{Max}$ & $\mathbf{n}$ & mean & SD & Min & Max \\
\hline \multicolumn{11}{|l|}{0.17} \\
\hline \multicolumn{11}{|l|}{0.33} \\
\hline \multicolumn{11}{|l|}{0.50} \\
\hline 1 & 1 & 129.0 & & & & & & & & \\
\hline \multicolumn{11}{|l|}{2} \\
\hline \multicolumn{11}{|l|}{3} \\
\hline \multicolumn{11}{|l|}{4} \\
\hline 5 & 1 & 199.5 & & & & & & & & \\
\hline 6 & 2 & 247.8 & & 237.5 & 258.0 & & & & & \\
\hline 7 & 1 & 249.5 & & & & & & & & \\
\hline \multicolumn{11}{|l|}{8} \\
\hline \multicolumn{11}{|l|}{9} \\
\hline \multicolumn{11}{|l|}{10} \\
\hline 11 & 4 & 350.1 & 36.4 & 302.0 & 380.5 & 20 & 383.7 & 24.5 & 342.0 & 439.0 \\
\hline 12 & 2 & 353.8 & 49.9 & 318.5 & 389.0 & 20 & 404.0 & 24.9 & 359.0 & 447.0 \\
\hline 13 & 1 & 396.0 & & & & 20 & 424.2 & 25.6 & 375.0 & 468.0 \\
\hline 14 & & & & & & 20 & 441.9 & 23.4 & 404.0 & 488.0 \\
\hline 15 & 6 & 391.2 & 14.3 & 376.0 & 413.5 & 20 & 455.1 & 24.1 & 415.0 & 503.0 \\
\hline 16 & 6 & 403.9 & 20.5 & 379.0 & 440.0 & 20 & 462.6 & 25.9 & 416.0 & 517.0 \\
\hline 17 & 2 & 432.2 & 18.7 & 419.0 & 445.5 & 20 & 466.4 & 26.6 & 416.0 & 522.0 \\
\hline 18 & 1 & 411.0 & & & & & & & & \\
\hline
\end{tabular}




\section{Appendix D. Femoral joint dimension prediction equations and scatter plots for} Later Stone Age juveniles
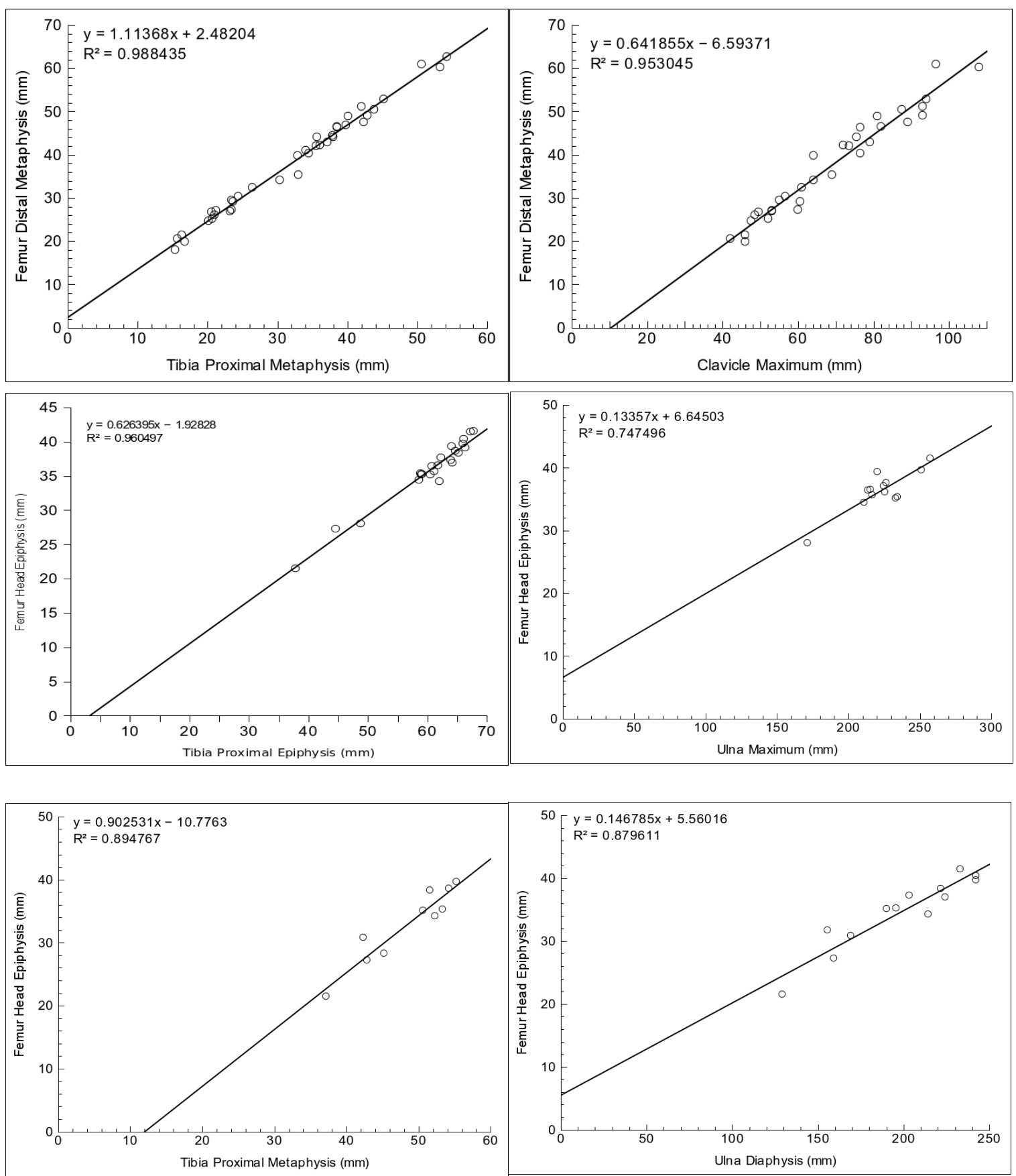


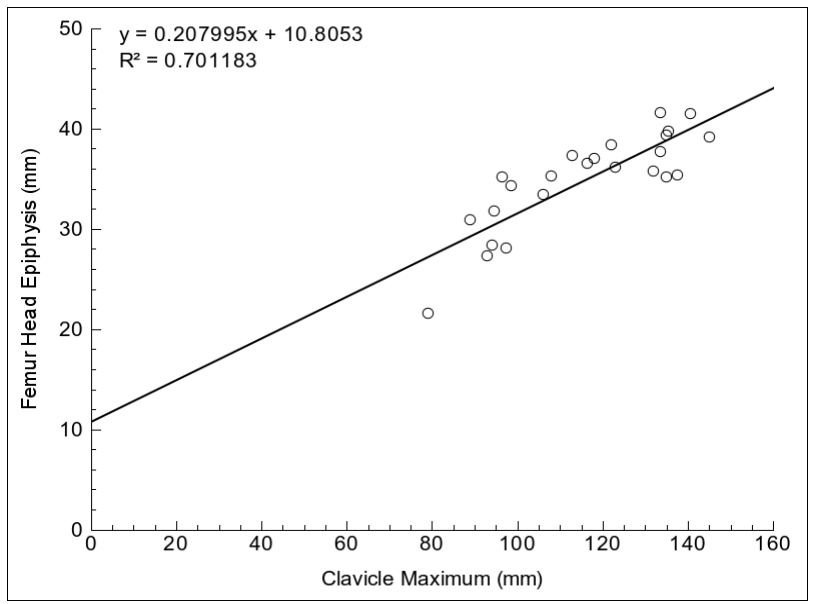




\section{Appendix E. Body size standardized skeletal growth profiles for Later Stone Age} juveniles
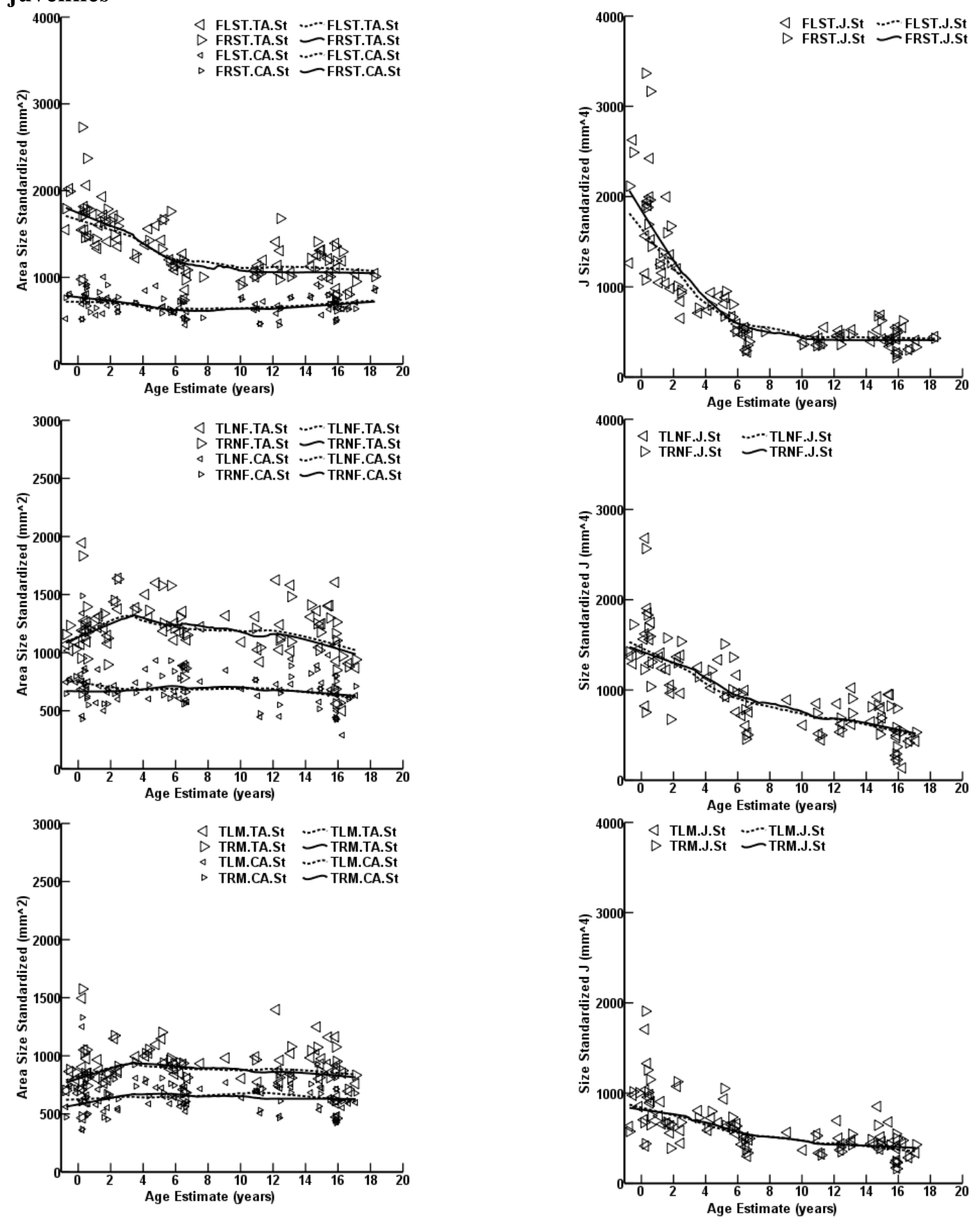

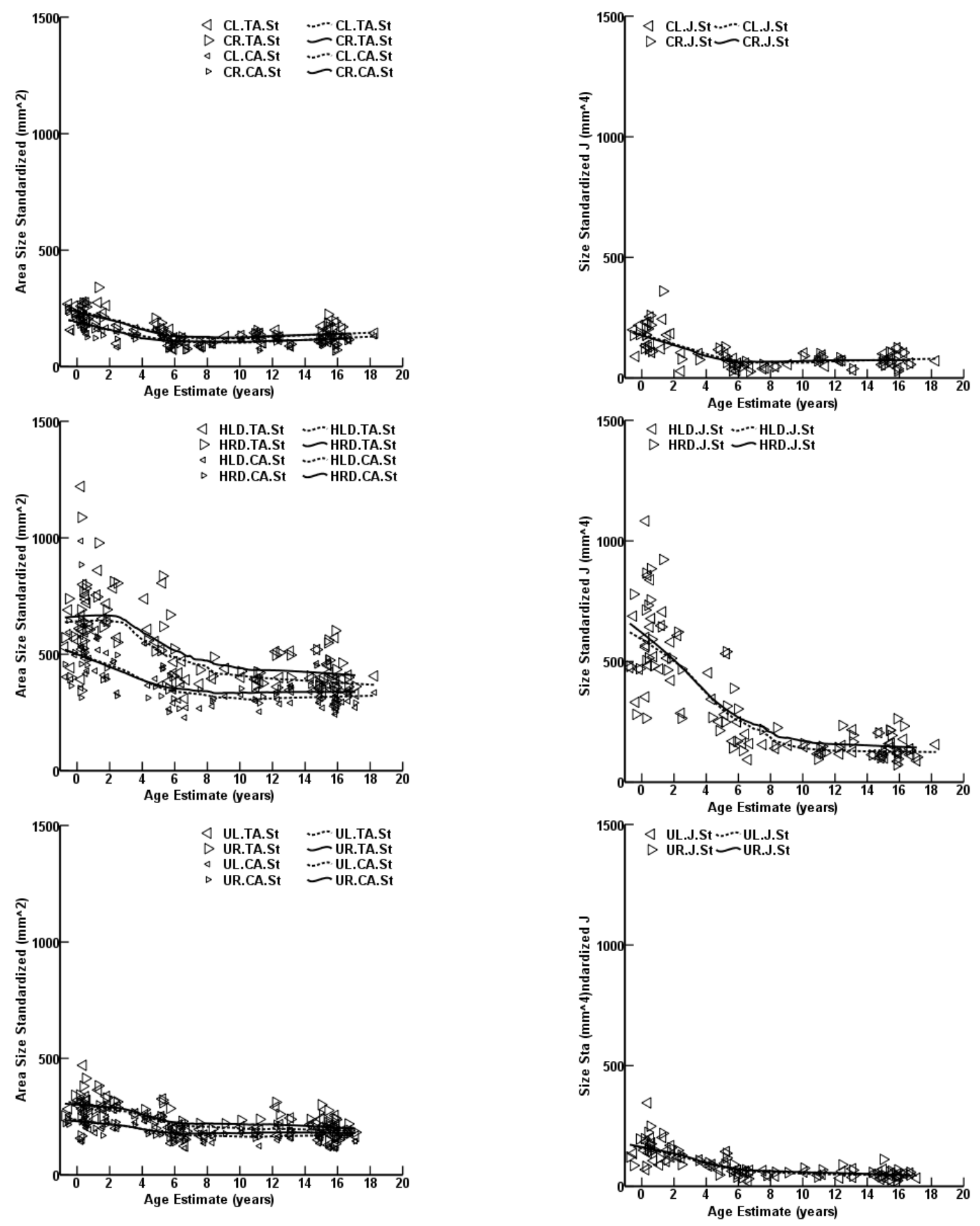